\title{
Osteological guide of songbirds from Central Europe
}

\author{
JENŐ (Eugen) KessLER
}

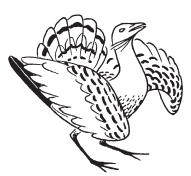

Jenő (Eugen) Kessler 2015. Osteological guide of songbirds from Central Europe. - Ornis Hungarica 23(2): 62-155.

Abstract The author provides an osteological guide to songbirds, based on 11 skeletal parts of 51 genera, at the genus level for ornithologists studying owl pellets, paleontologists and archaezoologists. The mandible, the coracoid bone, the scapula, the humerus, the ulna, the carpometacarp, the first phalanx bone of the second finger, the femur, the tibiotars, the tarsometatars and the claw bone are presented.

The morphological characteristics and method of measurement of the examined skeletal parts and the photographs of the appropriate bones are illustrated on 52 plates and 17 figures. The measurement data are also provided in 11 size tables. For every discussed bone, 3-6 characteristics are chosen, and their codes consist of 3-6 letters. In case of overlaps, dimensions are the determining factors, and for the humerus and the ulna, the ensemble of the two bone end pairs can be used.

Keywords: Central-Europe, guide, osteology, Paleornithology, archeozoology, Passerines

Összefoglalás A szerző 51 énekesmadár genus 11 vázrészének morfológiai jellemzőin alapuló csonthatározót állított össze a bagolyköpeteket vizsgáló ornitológusoknak, paleontológusoknak, archaeozoológusoknak ajánlva. A dolgozatban az alsó állkapocs (mandibula), a hollócsőrcsont (coracoideum), a lapocka (scapula), a felkarcsont (humerus) és a singcsont (ulna), a kézközépcsont (carpometacarpus), a kézujjperc (phalanx proximalis digiti majoris), a combcsont (femur), a lábszárcsont (tibiotarsus), a csüd (tarsometatarsus) és a karomcsont (phalanx ungularis) jellemzői találhatók.

A vizsgált vázrészek morfológiai jellegei, mérési módja és a csontok fényképe 52 táblaképen és 17 ábrán található. Tájékoztatási célból a mérési adatokat is közöljük 11 táblázatban. A határozó a kiválasztott morfológiai jellegek kódolásán alapszik. Minden tárgyalt csonton 3-6 jelleg került kiválasztásra, és ennek megfelelően a kódok 3-6 betüből állnak. Átfedések esetén a méretek az irányadóak, illetve a felkarcsontnál és a singcsontnál a két-két csontvég együttese használható.

Kulcsszavak: Közép-Európa, határozó, csonttani, paleornitológia, archeozoológia, énekesmadarak

Department of Paleontology, Eötvös Loránd University, 1117 Budapest, Pázmány Péter sétány 1/c, Hungary, e-mail: kessler_jeno@yahoo.com

\section{Introduction}

Despite the numerous songbird skeletal parts remaining in owl pellets, rocks from the Neogene period and at archaeological sites alike, the identification of these is still lagging behind all across Europe due to the lack of an ample comparative skeleton collection, and a relatively easy-to-use guidebook.
The current work wishes to aid this work by providing codes as well as images and measurement data.

The preparation of the guide was limited to the material of the recent osteological collection available in the Paleontological and Geological Department of the Hungarian Natural History Museum. There are only 48 (or 46-47) because not all genera had all the skeleton parts, or they were not in ample 
condition to photograph, hence the lower number of shown parts than the number of processed genera (51).

Only those skeletal parts are presented which have remained complete and which carry morphological characteristics that are characteristic at the genus level.

On the skull, only the tip of the mandibula, the proximal end of the coracoid and the scapula from the pectoral girdle are described. On the upper limb, the humerus, the ulna, the metacarpus bones (carpometacar$p u s$ ) and the first phalanx bone of the second finger (phalanx proximalis digiti majoris) completely, whereas on the lower limb, the femur, the tibiotarsus, the distal end of the tarsometatarsus and the claw (phalanx ungularis) are described. The point is that since both ends (epiphysis) of either a humerus or an ulna are enough for a definition, and often the ends surface separately or partially because the bone itself is broken, they both can be traced as parts of the same bone and had been coded as such.

In the case of other long bones, only the end part, which had been easier to define, was coded.

Other parts of the excessively fragile skull, vertebrae, ribs, the sternum, the pelvis (synsacrum), the clavicula, the radius, a few carpi (carpale) and the fibula (peroneum) are not presented here.

Since the largest family of the order of songbirds (Passeriformes), the Corvidae, is relatively well-known (Kessler \& Moldvai 1993, Tomek \& Bochenski 2000), genera belonging to here are not discussed. However, the Western Jackdaw (Corvus monedula) is used to illustrate anatomical and dimensional characteristics.

The distinctive feature of the guide lies in the method of coding. Each discussed skeletal part of each genus receives a 3-6-letter code, which can be used to identify the appropriate genus from the bone. The typical code types are presented for skeletal parts via tables and figures.

The guide is supplemented by 52 tables and 17 figures, with 4-4 tables for each discussed skeletal part. Eight of these are graphical illustrations (in case of the mandibula and scapula), while other bones are presented by original photographs. The preparatory state of the bones in the collection influenced the colour and sharpness of the photographs. The degreased skeletal parts appear more bright and sharp, while those remaining greased are less sharp and are darker. Colour and sharpness were modified by transforming the original colour image to greyscale.

Since more than half of the discussed 51 genera represent several species which primarily differ in size or color of their feathers, we also attach tables of dimensions for each skeletal part for information. The dimensions are taken from one specimen. In case of genera containing several species, the lower and upper limits of all the species are given.

From practical considerations we used the taxonomy after Cramp (1998). (Except of Lusciniola, wich was separated from Acrocephalus.)

Finally, it was not possible to show bones from the same sides of each skeletal part on the pictures.

\section{Method}

For every discussed skeletal part, the anatomical terminology (Baumel et al. 1979) and method of measurement (von den Driesch 1976, Gál 2002, Kessler 2013) of the bone in question is given, illustrated by the 
appropriate bone of the Western Jackdaw. Arrows indicate the coded characteristics.

For each anatomical character, a number of different types are differentiated, which received capitalized codes. We attempted to keep the number of codes at a minimum, since we had to take into account the individual differences and, in the case of genera featuring several species, the differences between them. This way we could make sure that even the more or less similar anatomical features can be classified easier and we could avoid subjective judgement.

Based on the characteristics used, we constructed a code consisting of 3-6 letters. The majority of these is typical for a single genera, but in a few cases, for more than one. In such cases, either the difference in size will define where they belong, or for similar sizes, the tables and figures provide guidance. (The tables with the bone sizes include genera with a single species, and genera with several species. In the first case, only a single size of the species is given. In the latter case, the limits of the smallest species and the biggest species from the genus are given.)

During genus identification, we create the code for the specific bone (or end of bone) and match it with the appropriate code in this guide. The images of genera with similar (but not identical) codes can give further help.

It is worth noting that the fewer of the characteristics are coded, the higher the chance of overlapping. Regarding overlapping of the humerus and ulna, we have to take into account the codes of both of the epiphyses.

Since for a definition of guaranteed accuracy the recent comparative osteological collection is absolutely required, this current guide can only serve information purposes.
Anatomical terminology (after: Milne-Edwards 1867-68, Fürbringer 1888, Lambrecht 1933, Ballmann 1966, Mourer-Chauviré 1975, Baumel et al. 1979, Gilbert et al. 1981, Cheneval 1983, Jánossy 1985, Moreno 1985, 1986, 1987, Cuisin 1989, Ujhelyi 1992, Kessler \& Moldvai 1993, Solti 1996, Tomek \& Bochenski 2000, Kessler 2013a). Plate with morphological characters (Figure 1).

\section{1. mandibula:}

a. rostrum mandibulae;

b. the immersed part of the rostrum;

c. ramus mandibulae;

\section{2. coracoideum:}

a. acrocoracoideum;

b. tuberculum brachiale (processus acrocoracoideus);

c. sulcus musculi supracoracoidei;

d. processus procoracoideus;

e. processus glenoidalis coracoidei;

\section{3. scapula:}

a. dorsal branch of the acromion;

b. lateral branch of the acromion;

c. the pit between the branches of the acromion;

d. acromion;

e. processus glenoidalis scapulae;

4. humerus (proximal epiphysis):

a. tuberculum ventrale;

b. crista bicipitalis;

c. the edge between crista bicipitalis and corpus humeri;

d. fossa pneumotricipitalis;

e. caput humeri;

f. crista deltopectoralis;

\section{5. humerus (distal epiphysis):}

a. tuberculum supracondylare ventrale; 


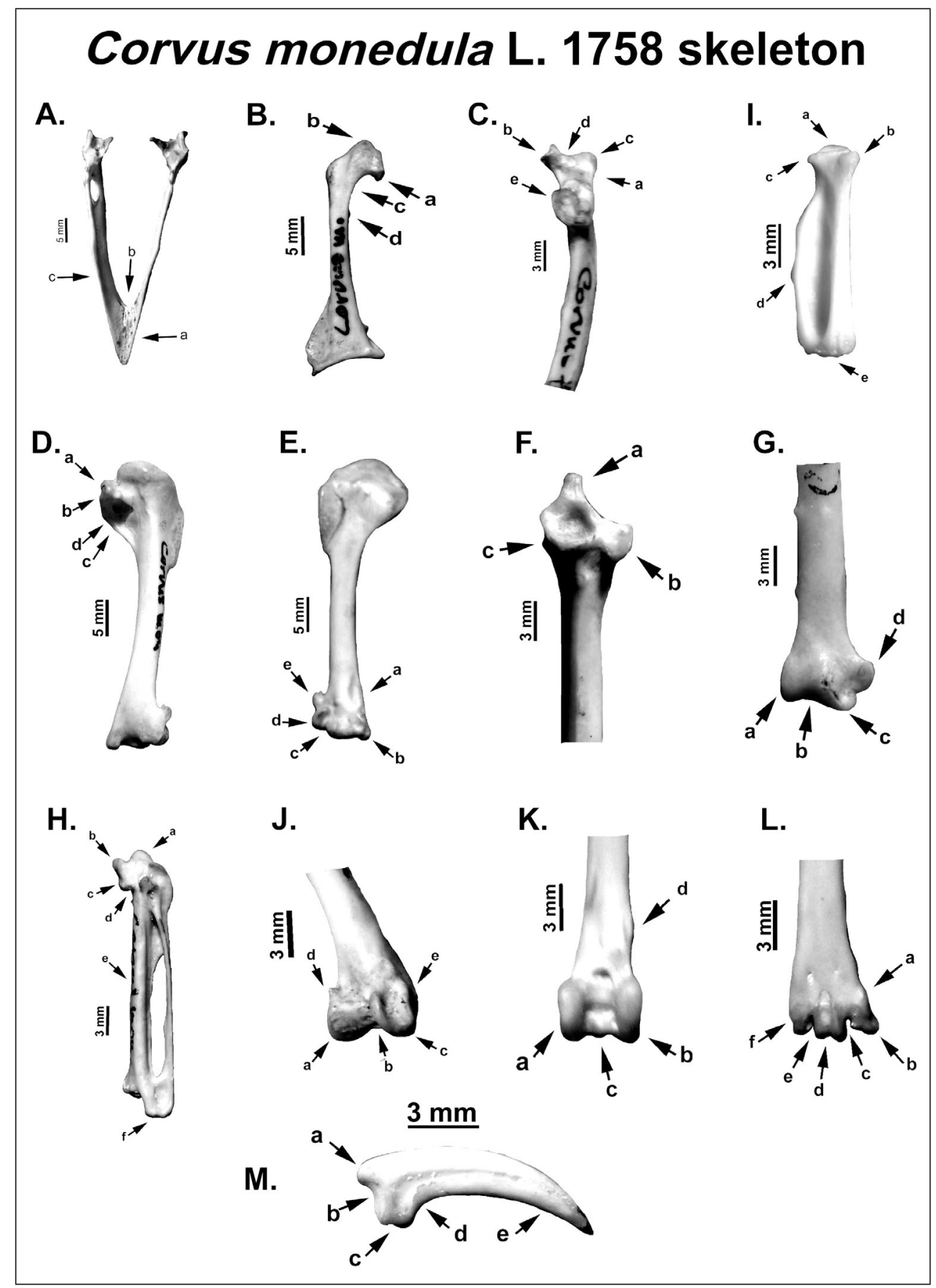

Figure 1 Morphological characters Corvus monedula L. 1758 bones;

(A - mandibula; B - coracoideum; C - scapula; D - humerus prox.; E - humerus dist.; F - ulna prox.; G - ulna dist.; H - carpometacarpus; I - phalanx prox. dig. maj.; J - femur dist.; $\mathrm{K}$ - tibiotarsus dist.; $\mathrm{L}$ - tarsometatarsus dist.; $\mathrm{M}$ - phalanx ungularis)

1. ábra Morfológiai jellegek Corvus monedula L. 1758 csontokon

(A - alsó állkapocs; B - hollócsőrcsont; C - lapocka; D - felkarcsont proximális epifízis; E - felkarcsont disztális epifízis; F - singcsont prox. epifízis; G - singcsont diszt. epifízis; H - kézközépcsont; I - szárny ujjperc; J - combcsont diszt. epifízis; K - lábszárcsont diszt. epifízis; L - csüd diszt. epifízis; $M$ - karomcsont) 

b. processus flexorius;
c. processus supracondylaris dorsalis;
d. incisura intercondylaris;
6. ulna (proximal epiphysis):
a. olecranon;
b. cotyla dorsalis;
c. tuberculum ligamentum collateralis ventralis;
7. $\quad$ ulna (distal epiphysis):
a. condylus dorsalis;
b. sulcus intercondylaris;
c. condylus ventralis;
d. tuberculum carpale;

\section{8. carpometacarpus:}

a. trochlea carpalis;

b. processus extensorius;

c. processus alularis;

d. fovea subalularis;

e. protuberantia metacarpale majus;

f. facies articularis digitale minor;

\section{9. phalanx proximalis digiti majoris:}
a. margo proximalis;
b. tuberculum ventralis;
c. tuberculum dorsalis;
d. margo dorsalis;
e. margo distalis;
10. femur (distal epiphysis):
a. condylus medialis;
b. sulcus intercondylaris;
c. condylus lateralis;
d. epicondylus medialis;
e. epicondylus lateralis;
11. tibiotarsus (distal epiphysis):
a. epicondylus lateralis;
b. epicondylus medialis;
c. incisura intercondylaris;
d. tuberositas retinaculi;
12. tarsometatarsus (distal epiphysis):
a. margo medialis;
b. trochlea metatarsi II.;
c. trochlea metatarsi III.;
d. trochlea metatarsi IV.;
e. incisura intertrochlearis medialis;
f. incisura intertrochlearis lateralis;

\section{3. phalanx ungularis:}

a. tuberculum extensorium;

b. cotyla articularis

c. tuberculum flexorium

d. the curvature of the margo plantaris

e. apex phalangis;

Measurement manner: (after: von den Driesch 1976, Gál 2002, Kessler 2013b)

Plate with measurement manner (Figure 2): TL - total length (a)

PL - partial length: mandibula - length of the rostrum (b), scapula - length of the proximal end (a)

$\mathrm{Bp}$ - breadth of the proximal end (b)

$\mathrm{Bc}$ - breadth of the corpus (c); mandibula breadth of the rostrum (a)

$\mathrm{Bd}$ - breadth of the distal end (d)

PB - partial breadth: mandibula - breadth of the ramus mandibulae (c)

\section{Abbrevations:}

No. sp - measured number of species LTB - coracoideum - length of the tuberculum brachialis (b)

\section{The codification}

1. Mandibula (Figure 4, Plate 1-4, Table 1)

\section{1. the pointed end of the rostrum man- dibulae:}

- A. short and medium width: Aegithalos; Bombycilla; 
- B. short and width: Carduelis; Coccothraustes; Chloris; Delichon; Emberiza; Hirundo; Loxia; Passer; Pinicola; Plectrophenax; Pyrrhula; Riparia; Serinus;

- C. medium long and width: Fringilla; Lanius; Muscicapa; Oriolus;

- D. medium long and medium width: Acrocephalus; Anthus; Calandrella; Cinclus; Eremophila; Erithacus; Hippolais; Lullula; Luscinia; Oenanthe; Parus; Phoenicurus; Phylloscopus; Prunella; Remiz; Saxicola; Sitta; Sturnus; Sylvia; Turdus;

- E. long and narrow: Certhia; Troglodytes;

- F. long and medium width: Alauda; Cettia; Galerida; Locustella; Melanocorypha; Monticola; Motacilla;

\section{2. the immersed part of the rostrum:}

- A. semicircular: Aegithalos; Bombycilla; Carduelis; Emberiza; Hippolais; Muscicapa; Oenanthe; Sylvia;

- B. semi-circle in the middle with small tip: Anthus; Delichon; Monticola; Motacilla; Parus;

- C. ovoid: Alauda; Calandrella; Cinclus; Eremophila; Galerida; Hirundo; Lanius; Melanocorypha;

- D. ovoid with small tip: Locustella; Lullula; Riparia; Troglodytes;

- E. pointed: Acrocephalus; Certhia; Sitta;

- F. semi-circle with small recess: Erithacus; Oriolus; Prunella;

- G. shouldered recess: Cettia; Coccothraustes; Loxia; Phoenicurus; Phylloscopus; Saxicola; Sturnus; Turdus;

- H. wide semi-circle: Luscinia; Muscicapa; Fringilla; Plectrophenax; Remiz;

- I. wide semi-circle with small tip: Chloris; Passer; Pinicola; Pyrrhula; Serinus;

\section{3. the form of the ramus mandibulae:}

- A. straight: Acrocephalus; Alauda; Calandrella; Certhia; Cettia; Chloris;
Cinclus; Emberiza; Galerida; Hippolais; Locustella; Lullula; Luscinia; Monticola; Oenanthe; Parus; Phylloscopus; Prunella; Saxicola; Sitta; Sturnus; Sylvia; Troglodytes;

- B. slightly protruding above: Aegithalos; Anthus; Bombycilla; Eremophila; Motacilla, Plectrophenax; Remiz;

- C. wide or medium protruding above: Delichon; Erithacus; Lanius; Muscicapa; Oriolus; Phoenicurus; Plectrophenax;

- D. strongly protruding above: Hirundo; Riparia; Melanocorypha;

- E. slightly protruding below: Carduelis; Fringilla; Passer; Serinus;

- F. strongly protruding below: Chloris; Coccothraustes; Loxia; Pinicola; Pyrrhula;

Alaudidae: Alauda FCA; Calandrella DCA; Eremophila DCB; Galerida FCA; Lullula DDA; Melanocorypha DED;

Hirundinidae: Delichon BBC; Hirundo $\mathrm{BCD}$; Riparia BDD;

Paridae: Aegithalos AAB; Parus DBA; Remiz DHB;

Certhiidae: Certhia EEA;

Sittidae: Sitta DEA;

Muscicapidae: Erithacus DFC; Luscinia DHA; Monticola FBA; Muscicapa CAC, Oenanthe DAA; Phoenicurus DGC; Saxicola DGA;

Turdidae: Turdus DGA;

Oriolidae: Oriolus CFC;

Sylviidae: Acrocephalus DEA; Cettia FGA; Hippolais DAA; Locustella FDA; Phylloscopus CGA; Sylvia CAA;

Motacillidae: Anthus DBB; Motacilla FBB;

Bombycillidae: Bombycilla AAB;

Troglodytidae: Troglodytes EDA;

Cinclidae: Cinclus DCA;

Prunellidae: Prunella DFA;

Laniidae: Lanius CCC; 
Sturnidae: Sturnus DGA;

Passeridae: Passer BIE;

Fringillidae: Carduelis BAE; Chloris BIF; Coccothraustes BGF; Fringilla CHE; Loxia BGF; Pinicola BIF; Pyrrhula BIF; Serinus BIE;

Emberizidae: Emberiza BAA; Plectrophenax BHB;

AAB: Aegithalos; Bombycilla;

BAA: Emberiza;

BAE: Carduelis;

BBC: Delichon;

BCD: Hirundo;

BDD: Riparia;

BGF: Coccothraustes; Loxia;

BHB: Plectrophenax;

BIE: Passer; Serinus;

BIF: Chloris; Pinicola; Pyrrhula;

CAC: Muscicapa;

CCC: Lanius;

CFC: Oriolus;

CHE: Fringilla;

DAA: Hippolais; Oenanthe; Sylvia;

DBA: Parus,

DBB: Anthus;

DCA: Calandrella; Cinclus;

DCB: Eremophila;

DDA: Lullula;

DEA: Acrocephalus; Sitta;

DED: Melanocorypha;

DFA: Prunella;

DFC: Erithacus;

DGA: Phylloscopus; Saxicola; Sturnus;

Turdus;

DGC: Phoenicurus;

DHA: Luscinia;

DHB: Remiz;

EDA: Troglodytes;

EEA: Certhia;

FBA: Monticola;

FBB: Motacilla;

FCA: Alauda; Galerida;
FDA: Locustella;

FGA: Cettia;

2. Coracoideum (Figure 5, Plate 5-8, Table 2)

2.1. the processus acrocoracoideus (tuberculum brachiale):

- A. long and blunt: Aegithalos; Certhia; Emberiza; Hippolais; Luscinia; Lusciniola; Muscicapa; Phoenicurus; Phylloscopus; Regulus; Saxicola; Sitta;

- B. long and pointed: Acrocephalus; Cettia; Erithacus; Oenanthe; Oriolus; Sylvia;

- C. short and blunt: Alauda; Anthus; Bombycilla; Calandrella; Carduelis; Chloris; Coccothraustes; Delichon; Galerida; Lanius; Locustella; Loxia; Lullula; Melanocorypha; Monticola; Motacilla; Parus; Passer; Pinicola; Pyrrhula; Remiz; Riparia; Sturnus; Troglodytes;

- D. short and pointed: Fringilla; Hirundo; Panurus; Plectrophenax; Prunella; Turdus;

\section{2. the acrocoracoideum:}

- A. symmetrical: Aegithalos; Alauda; Anthus; Carduelis; Chloris; Coccothraustes; Emberiza; Erithacus; Galerida; Loxia; Luscinia; Lullula; Melanocorypha; Monticola; Motacilla; Muscicapa; Oenanthe; Panurus; Pinicola; Phylloscopus; Plectrophenax; Pyrrhula; Remiz; Riparia; Sitta; Sturnus;

- B. assymmetric: Acrocephalus; Bombycilla; Calandrella; Certhia; Cettia; Delichon; Fringilla; Hippolais; Hirundo; Lanius; Locustella; Lusciniola; Oriolus; Parus; Passer; Phoenicurus; Prunella; Regulus; Saxicola; Sylvia; Troglodytes; Turdus; 


\section{3. the sulcus musculi supracoracoidei:}

- A. symmetrically semicircular: Acrocephalus; Certhia; Erithacus; Lanius; Melanocorypha; Monticola; Muscicapa; Oenanthe; Pyrrhula; Turdus;

- B. asymmetrically semicircular: Aegithalos; Bombycilla; Carduelis; Cettia; Chloris; Coccothraustes; Delichon; Galerida; Hippolais; Hirundo; Luscinia; Lusciniola; Oriolus; Panurus; Parus; Phoenicurus; Phylloscopus; Regulus; Remiz; Riparia; Saxicola; Sitta; Sturnus; Sylvia;

- C. flattened: Alauda; Anthus; Calandrella; Emberiza; Fringilla; Locustella; Loxia; Lullula; Motacilla; Passer; Pinicola; Plectrophenax; Prunella; Troglodytes;

\section{4. the processus procoracoideus:}

- A. missing: Bombycilla; Cettia; Delichon; Galerida; Hippolais; Hirundo; Luscinia; Lusciniola; Motacilla; Panurus; Parus; Phylloscopus; Remiz; Riparia;

- B. slightly overhanging: Acrocephalus; Aegithalos; Alauda; Carduelis; Chloris; Lanius; Locustella; Melanocorypha; Passer; Phoenicurus; Prunella; Regulus; Sitta; Sylvia;

- C. strongly protruding: Anthus; Calandrella; Certhia; Coccothraustes; Emberiza; Erithacus; Fringilla; Loxia; Lullula; Monticola; Muscicapa; Oenanthe; Oriolus; Pinicola; Plectrophenax; Pyrrhula; Saxicola; Sturnus; Troglodytes; Turdus;

Alaudidae: Alauda CACB; Calandrella CBCC; Galerida CABA; Lullula CACC; Melanocorypha CAAB;

Hirundinidae: Delichon CBBA; Hirundo DBBA; Riparia CABA;

Paridae: Aegithalos AABB; Panurus DABA; Parus CBBA; Remiz CABA;

Certhiidae: Certhia ABAC;

Sittidae: Sitta AABB;
Muscicapidae: Erithacus BAAC; Luscinia AABA; Monticola CAAC; Muscicapa AAAC, Oenanthe BAAC; Phoenicurus ABBB; Saxicola ABBC;

Turdidae: Turdus DBAC;

Oriolidae: Oriolus BBBC;

Sylviidae: Acrocephalus BBAB; Cettia BBBA; Hippolais ABBA; Locustella CBCB; Lusciniola ABBA; Phylloscopus AABA; Regulus ABBB; Sylvia BBBB;

Motacillidae: Anthus CACC; Motacilla CACA;

Bombycillidae: Bombycilla CBBA;

Troglodytidae: Troglodytes CBCC;

Prunellidae: Prunella DBCB;

Laniidae: Lanius CBAB;

Sturnidae: Sturnus CABC;

Passeridae: Passer CBCB;

Fringillidae: Carduelis CABB; Chloris CABB; Coccothraustes CABC; Fringilla DBCC; Loxia CACC; Pinicola CACC; Pyrrhula CAAC;

Emberizidae: Emberiza AACC; Plectrophenax DACC;

AAAC: Muscicapa;

AABA: Luscinia; Phylloscopus;

AABB: Aegithalos; Sitta;

AACC: Emberiza;

ABAC: Certhia;

ABBA: Hippolais; Lusciniola;

ABBB: Phoenicurus; Regulus;

ABBC: Saxicola;

BAAC: Erithacus; Oenanthe;

BBAB: Acrocephalus;

BBBA: Cettia;

BBBB: Sylvia;

BBBC: Oriolus;

CAAB: Melanocorypha;

CAAC: Monticola; Pyrrhula;

CABA: Galerida; Riparia; Remiz;

CABB: Carduelis; Chloris;

CABC: Coccothraustes; Sturnus; 
CACA: Motacilla;

CACB: Alauda;

CACC: Anthus; Loxia; Lullula; Pinicola;

CBBA: Bombycilla; Delichon; Parus;

CBCB: Locustella; Passer;

CBCC: Calandrella;

DABA: Panurus;

DACC: Plectrophenax;

DBAC: Turdus;

DBCB: Prunella;

DBCC: Fringilla;

3. Scapula (Figure 6, Plate 9-12, Table 3)

3.1. the length of the branches of acromion:

- A. symmetrically (equal): Acrocephalus; Anthus; Calandrella; Carduelis; Certhia; Cettia; Coccothraustes; Delichon; Emberiza; Hippolais; Locustella; Lullula; Lusciniola; Monticola; Motacilla; Oenanthe; Parus; Passer; Phylloscopus; Pyrrhula; Sturnus; Sylvia; Turdus;

- B. asymmetrically (unequal): Alauda; Bombycilla; Chloris; Eremophila; Erithacus; Fringilla; Galerida; Hirundo; Lanius; Loxia; Luscinia; Melanocorypha; Muscicapa; Oriolus; Panurus; Phoenicurus; Pinicola; Plectrophenax; Prunella; Remiz; Riparia; Saxicola; Serinus; Sitta; Troglodytes;

3.2. the shape of the branches of acromion:

- A. symmetrically (equal): Acrocephalus; Carduelis; Coccothraustes; Delichon; Emberiza; Erithacus; Hippolais; Lusciniola; Motacilla; Muscicapa; Passer; Phylloscopus; Turdus;

- B. asymmetrically (unequal): Alauda; Anthus; Bombycilla; Calandrella; Certhia; Cettia; Chloris; Eremophila; Fringilla; Galerida; Hirundo; Lanius; Locustella; Loxia; Lullula; Luscinia; Melanocorypha; Monticola; Oenanthe;
Oriolus; Panurus; Parus; Phoenicurus; Pinicola; Plectrophenax; Prunella; Pyrrhula; Remiz; Riparia; Saxicola; Serinus; Sitta; Sturnus; Sylvia; Troglodytes;

\section{3. the apex dorsalis (dorsal branch):}

- A. short and blunted: Acrocephalus; Certhia; Cettia; Delichon; Erithacus; Hirundo; Locustella; Panurus; Plectrophenax; Riparia; Sitta;

- B. short and pointed: Serinus; Troglodytes;

- C. long and blunted: Anthus; Carduelis; Coccothraustes; Emberiza; Eremophila; Fringilla; Hippolais; Lanius; Loxia; Lusciniola; Melanocorypha; Motacilla; Muscicapa; Oenanthe; Oriolus; Parus; Passer; Phylloscopus; Pinicola; Prunella; Pyrrhula; Sturnus; Turdus;

- D. long and pointed: Bombycilla; Calandrella; Chloris; Galerida; Monticola; Phoenicurus; Saxicola; Sylvia;

- E. with a cut-off end: Alauda; Lullula; Luscinia; Remiz;

\section{4. the apex lateralis (lateral branch):}

- A. short and blunted: Certhia; Delichon; Erithacus; Galerida; Lanius; Melanocorypha; Loxia; Oriolus; Phoenicurus; Pinicola; Plectrophenax; Saxicola; Troglodytes;

- B. short and pointed: Bombycilla; Cettia; Hirundo; Locustella; Remiz;

- C. long and blunted: Acrocephalus; Alauda; Anthus; Calandrella; Carduelis; Coccothraustes; Emberiza; Eremophila; Fringilla; Hippolais; Lullula; Luscinia; Lusciniola; Motacilla; Monticola; Muscicapa; Oenanthe; Panurus; Parus; Passer; Phylloscopus; Pyrrhula; Riparia; Sylvia; Sturnus; Serinus; Turdus;

- D. long and pointed: Chloris; Sitta; 


\section{5. the pit between the branches:}

- A. deep and symmetrical: Alauda; Bombycilla; Calandrella; Carduelis; Chloris; Coccothraustes; Emberiza; Fringilla; Lanius; Lullula; Melanocorypha; Motacilla; Muscicapa; Oenanthe; Oriolus; Parus; Passer; Remiz; Sylvia; Turdus;

- B. deep and unsymmetrical: Anthus; Eremophila; Phoenicurus; Pinicola; Serinus; Sturnus;

- C. shallow and symmetrical: Acrocephalus; Cettia; Delichon; Erithacus; Galerida; Hippolais; Hirundo; Locustella; Luscinia; Lusciniola; Monticola; Phylloscopus; Plectrophenax; Pyrrhula; Riparia; Troglodytes;

- D. shallow and unsymmetrical: Certhia; Loxia; Panurus; Prunella; Saxicola; Sitta;

Alaudidae: Alauda BBECA; Calandrella ABDCA; Eremophila BBCCB; Galerida BBDAC; Lullula ABECA; Melanocorypha BBCAA;

Hirundinidae: Delichon AAAAC; Hirundo BBABC; Riparia BBACC

Paridae: Panurus BAACD; Parus

ABCCA; Remiz BBEBA;

Certhiidae: Certhia ABAAD;

Sittidae: Sitta BBADD;

Muscicapidae: Erithacus BAAAC; Luscinia BBECC; Monticola ABDCC; Muscicapa BACCA, Oenanthe ABCCA; Phoenicurus BBDAB; Saxicola BBDAD;

Turdidae: Turdus AACCA;

Oriolidae: Oriolus BBCAA;

Sylviidae: Acrocephalus AAACC; Cettia ABABC; Hippolais AACCC; Locustella ABABC; Lusciniola AACCC; Phylloscopus AACCC; Sylvia ABDCA;

Motacillidae: Anthus ABCCB; Motacilla AACCA;

Bombycillidae: Bombycilla BBDBA; Troglodytidae: Troglodytes BBBAC;
Prunellidae: Prunella BBCAD;

Laniidae: Lanius BBCAA;

Sturnidae: Sturnus ABCCB;

Passeridae: Passer AACCA;

Fringillidae: Carduelis AACCA; Chloris BBDDA; Coccothraustes AACCA; Fringilla BBCCA; Loxia BBCAD; Pinicola BBCAB; Pyrrhula ABCCC; Serinus BBBCB;

Emberizidae: Emberiza AACCA; Plectrophenax BBAAC;

AAAAC: Delichon;

AACCA: Carduelis; Coccothraustes; Emberiza; Motacilla; Passer; Turdus;

AAACC: Acrocephalus;

AACCC: Hippolais; Lusciniola; Phylloscopus;

ABAAD: Certhia;

ABABC: Cettia; Locustella;

ABBAB: Serinus;

ABCCA: Emberiza; Oenanthe; Parus;

ABCCB: Anthus; Sturnus;

ABCCC: Pyrrhula;

ABDCA: Calandrella; Sylvia;

ABDCC: Monticola;

ABECA: Lullula;

BAAAC: Erithacus;

BACCA: Muscicapa;

BBAAC: Plectrophenax;

BBABC: Hirundo;

BBACB: Anthus;

BBACB: Loxia;

BBACC: Riparia;

BBACD: Panurus;

BBADD: Sitta;

BBBAC: Troglodytes:

BBBCB: Serinus;

BBBCD: Saxicola;

BBCAA: Lanius; Melanocorypha; Oriolus;

BBCAB: Pinicola;

BBCAD: Loxia; Prunella;

BBCCA: Fringilla; 
BBCCB: Eremophila;

BBDAB: Phoenicurus;

BBDAC: Galerida;

BBDAD: Saxicola;

BBDBA: Bombycilla;

BBDDA: Chloris;

BBEBA: Remiz;

BBECA: Alauda;

BBECC: Luscinia;

4. Humerus (proximal epiphysis) (Figure 7, Plate 13-16, Table 4)

\section{1. the tuberculum ventrale:}

- A. strongly protruding: Alauda; Bombycilla; Certhia; Cettia; Cinclus; Coccothraustes; Delichon; Erithacus; Fringilla; Galerida; Hirundo; Loxia; Lullula; Luscinia; Lusciniola; Monticola; Motacilla; Muscicapa; Oenanthe; Oriolus; Passer; Phoenicurus; Phylloscopus; Pinicola; Pyrrhula; Riparia; Saxicola; Serinus;

- B. poorly protruding: Acrocephalus; Aegithalos; Anthus; Carduelis; Chloris; Emberiza; Hippolais; Lanius; Locustella; Melanocorypha; Panurus; Parus; Prunella; Sitta; Sturnus; Sylvia; Troglodytes; Turdus;

\section{2. the projection of the crista bicipitalis:}

- A. prominent and rounded: Aegithalos; Anthus; Emberiza; Erithacus; Galerida; Hippolais; Lullula; Passer; Phylloscopus; Pinicola; Prunella; Pyrrhula; Riparia; Serinus; Sitta; Sturnus; Troglodytes;

- B. prominent and pointed: Fringilla; Lusciniola;

- C. truncated: Acrocephalus; Carduelis; Certhia; Cettia; Chloris; Cinclus; Coccothraustes; Delichon; Hirundo; Lanius; Lanius; Locustella; Loxia; Oriolus; Parus; Sylvia; Turdus;
- D. rounded and not prominent: Bombycilla; Lanius; Lullula; Luscinia; Melanocorypha; Motacilla; Muscicapa; Panurus; Saxicola;

- E. slightly pointed distally: Alauda; Monticola; Oenanthe; Phoenicurus; Turdus;

4.3. the distal edge of the crista bicipitalis:

- A. flatly curved: Alauda; Bombycilla; Chloris; Cinclus; Delichon; Fringilla; Loxia; Luscinia; Panurus; Passer; Pinicola; Prunella; Riparia; Sturnus;

- B. strongly curved: Acrocephalus; Aegithalos; Anthus; Carduelis; Certhia; Cettia; Chloris; Coccothraustes; Emberiza; Erithacus; Galerida; Hippolais; Hirundo; Lanius; Locustella; Lullula; Lusciniola; Melanocorypha; Monticola; Motacilla; Muscicapa; Oenanthe; Oriolus; Parus; Phoenicurus; Phylloscopus; Pyrrhula; Saxicola; Serinus; Sitta; Sylvia; Troglodytes; Turdus;

\section{4. the fossa pneumotricipitalis:}

- A. split: Acrocephalus; Alauda; Bombycilla; Certhia; Cettia; Cinclus; Delichon; Galerida; Hirundo; Hippolais; Lanius; Locustella; Lullula; Lusciniola; Melanocorypha; Monticola; Muscicapa; Oenanthe; Oriolus; Passer; Phoenicurus; Phylloscopus; Prunella; Riparia; Saxicola; Sturnus; Sylvia; Turdus;

- B. no split: Aegithalos; Anthus; Carduelis; Chloris; Coccothraustes; Emberiza; Erithacus; Fringilla; Loxia; Luscinia; Motacilla; Panurus; Parus; Pinicola; Pyrrhula; Serinus; Sitta; Troglodytes;

\footnotetext{
Alaudidae: Alauda AEAA; Galerida AABA; Lullula ADBA; Melanocorypha BDBA;

Hirundinidae: Delichon ACAA; Hirundo ACBA; Riparia AAAA;
} 
Paridae: Aegithalos BABB; Panurus BDAB; Parus BCBB;

Certhiidae: Certhia ACBA;

Sittidae: Sitta BABB;

Muscicapidae: Erithacus AABB; Luscinia ADAB; Monticola AEBA; Muscicapa ADBA; Oenanthe AEBA; Phoenicurus AEBA; Saxicola ADBA;

Turdidae: Turdus BEBA;

Oriolidae: Oriolus ACBA;

Sylviidae: Acrocephalus BCBA; Cettia ACBA; Hippolais BABA; Locustella BCBA; Lusciniola ABBA; Phylloscopus AABA; Sylvia BCBA;

Motacillidae: Anthus BABB; Motacilla ADBB;

Bombycillidae: Bombycilla ADAA;

Troglodytidae: Troglodytes BABB;

Cinclidae: Cinclus ACBA:

Prunellidae: Prunella BAAA;

Laniidae: Lanius BCBA;

Sturnidae: Sturnus BAAA;

Passeridae: Passer AAAA;

Fringillidae: Carduelis BCBB; Chloris ACAB; Coccothraustes ACBB; Fringilla ABAB; Loxia ACAB; Pinicola AAAB; Pyrrhula AABB; Serinus AABB;

Emberizidae: Emberiza BABB;

AAAA: Riparia; Passer;

AAAB: Pinicola;

AABA: Galerida; Phylloscopus;

AABB: Erithacus; Pyrrhula; Serinus;

ABAB: Fringilla;

ABBA: Lusciniola;

ACAA: Certhia; Coccothraustes; Delichon;

ACAB: Chloris; Loxia;

ACBA: Cettia; Certhia; Cinclus: Hirundo; Oriolus;

ACBB: Coccothraustes;

ADAA: Bombycilla;

ADAB: Luscinia;
ADBA: Lullula; Muscicapa; Saxicola;

ADBB: Motacilla;

AEAA: Alauda;

AEBA: Monticola; Oenanthe; Phoenicurus;

BAAA: Prunella; Sturnus;

BAAB: Sitta;

BABA: Emberiza; Hippolais;

BABB: Aegithalos; Anthus; Emberiza; Sitta; Troglodytes;

BCAA: Parus;

BCBA: Acrocephalus; Lanius; Locustella; Sylvia;

BCBB: Carduelis; Parus;

BDAB: Panurus;

BDBA: Melanocorypha;

BEBA: Turdus;

5. Humerus (distal epiphysis) (Figure 8, Plate 17-20, Table 4)

5.1. the tuberculum supracondylare ventrale:

- A. protuberant: Acrocephalus; Aegithalos; Bombycilla; Carduelis; Certhia; Cettia; Chloris; Cinclus; Coccothraustes; Delichon; Emberiza; Erithacus; Hippolais; Hirundo; Loxia; Lullula; Melanocorypha; Monticola; Parus; Parus; Passer; Pinicola; Riparia; Saxicola; Serinus; Sitta; Sturnus; Troglodytes;

- B. not prominent: Alauda; Anthus; Fringilla; Galerida; Lanius; Locustella; Luscinia; Lusciniola; Motacilla; Muscicapa; Oenanthe; Oriolus; Panurus; Phoenicurus; Phylloscopus; Prunella; Pyrrhula; Sylvia; Turdus;

\section{2. the processus flexorius:}

- A. slightly overhanging: Alauda; Carduelis; Cinclus; Galerida; Emberiza; Lullula; Lusciniola; Sitta; 
- B. strongly overhanging with rounded ends: Anthus; Bombycilla; Certhia; Chloris; Coccothraustes; Emberiza; Fringilla; Locustella; Monticola; Motacilla; Muscicapa; Oriolus; Panurus; Passer; Phoenicurus; Prunella; Pyrrhula; Riparia; Saxicola; Serinus; Sturnus; Troglodytes;

- C. strongly overhanging with severed ends: Acrocephalus; Aegithalos; Cettia; Erithacus; Hippolais; Lanius; Loxia; Luscinia; Oenanthe; Parus; Pinicola; Sylvia; Turdus;

- D. markedly protruding conically: Melanocorypha; Phylloscopus;

- E. asymmetrical strongly protruding: Delichon; Hirundo; Riparia;

\section{3. the incisura intercondylaris:}

- A. concave and wide: Alauda; Cinclus: Coccothraustes; Delichon; Emberiza; Galerida; Lusciniola; Melanocorypha; Phoenicurus;

- B. slightly concave: Anthus; Chloris; Fringilla; Lanius; Lullula; Luscinia; Monticola; Motacilla; Muscicapa; Oenanthe; Oriolus; Panurus; Parus; Passer; Prunella; Pyrrhula; Saxicola; Serinus; Sitta; Sylvia; Troglodytes;

- C. pointed and wide: Acrocephalus; Aegithalos; Bombycilla; Carduelis; Certhia; Cettia; Erithacus; Hippolais; Hirundo; Locustella; Loxia; Phylloscopus; Pinicola; Riparia; Sturnus; Turdus;

\section{4. the condylus dorsalis:}

- A. rounded: Alauda; Anthus; Bombycilla; Carduelis; Certhia; Cettia; Chloris; Cinclus; Coccothraustes; Emberiza; Erithacus; Fringilla; Galerida; Hirundo; Lanius; Locustella; Loxia; Lullula; Luscinia; Lusciniola; Lusciniola; Melanocorypha; Monticola; Motacilla; Muscicapa; Oenanthe; Panurus; Parus;
Passer; Phoenicurus; Phylloscopus; Pinicola; Prunella; Pyrrhula; Saxicola; Serinus; Sturnus; Sylvia; Troglodytes;

- B. asymmetric: Acrocephalus; Aegithalos; Delichon; Hippolais; Oriolus; Riparia; Sitta; Turdus;

\section{5. the processus supracondylaris dorsalis:}

- A. single and slightly protruding: Prunella;

- B. single and strongly protruding: Cinclus: Delichon; Lanius; Locustella; Loxia; Melanocorypha; Muscicapa; Panurus; Serinus; Troglodytes; Turdus;

- C. two-pronged equal: Aegithalos; Alauda; Certhia;

- D. two-pronged unequal: Acrocephalus; Anthus; Bombycilla; Carduelis; Cettia; Chloris; Coccothraustes; Emberiza; Erithacus; Fringilla; Galerida; Hippolais; Hirundo; Lullula; Luscinia; Lusciniola; Monticola; Motacilla; Oenanthe; Oriolus; Parus; Passer; Phylloscopus; Phoenicurus; Pinicola; Pyrrhula; Riparia; Saxicola; Sitta; Sturnus; Sylvia;

Alaudidae: Alauda BAAAC; Galerida BAAAD; Lullula AAABD; Melanocorypha ADAAB;

Hirundinidae: Delichon AEABB; Hirundo AECAD; Riparia AECBD;

Paridae: Aegithalos ACCBC; Panurus BBBAB; Parus ACBAD;

Certhiidae: Certhia ABCAC;

Sittidae: Sitta AABBD;

Muscicapidae: Erithacus ABCAD; Luscinia BCBAD; Monticola ABBAD; Muscicapa BBBAB; Oenanthe BCBAD; Phoenicurus BBAAD; Saxicola ABBAD;

Turdidae: Turdus BCCBB; Oriolidae: Oriolus BBBBD;

Sylviidae: Acrocephalus ACCBD; Cettia ACCAD; Hippolais ACCBD; Locustella 
BBCAB; Lusciniola BAAAD; Phylloscopus BDCAD; Sylvia BCBAD;

Motacillidae: Anthus BBBAD; Motacilla BBBAD;

Bombycillidae: Bombycilla ABCAD;

Troglodytidae: Troglodytes ABBAB;

Cinclidae: Cinclus AAAAB;

Prunellidae: Prunella BBBAA;

Laniidae: Lanius BCBAB;

Sturnidae: Sturnus ABCAD;

Passeridae: Passer ABBAD;

Fringillidae: Carduelis AACAD; Chloris ABBAD; Coccothraustes ABAAD; Fringilla BBBAD; Loxia ACCAB; Pinicola ACCAD; Pyrrhula BBBAD; Serinus ABBAB;

Emberizidae: Emberiza ABAAD;

AAAAB: Cinclus;

AAABD: Lullula;

AABBD: Sitta;

ABAAD: Coccothraustes; Emberiza;

ABBAB: Serinus; Troglodytes;

ABBAD: Chloris; Monticola; Passer; Saxicola;

ABCAC: Certhia;

ABCAD: Bombycilla; Erithacus; Sturnus;

ACBAD: Parus;

ACCAB: Loxia;

ACCAD: Cettia; Pinicola;

ACCBC: Aegithalos;

ACCBD: Acrocephalus; Hippolais;

ADAAB: Melanocorypha;

AEABB: Delichon;

AECAD: Hirundo;

AECBD: Riparia;

BAAAC: Alauda;

BAAAD: Galerida; Lusciniola;

BBAAD: Phoenicurus;

BBBAA: Prunella;

BBBAB: Muscicapa; Panurus;

BBBAD: Anthus; Fringilla; Motacilla; Pyrrhula;
BBBBD: Oriolus,

BBCAB: Locustella;

BCBAB: Lanius;

BCBAD: Luscinia; Oenanthe; Sylvia;

BCCBB: Turdus;

BDCAD: Phylloscopus;

6. Ulna (proximal epiphysis) (Figure 9, Plate 21-24, Table 5)

\section{1. the olecranon:}

- A. short and pointed: Alauda; Certhia; $\mathrm{Hi}$ rundo; Locustella; Muscicapa; Pyrrhula;

- B. short and blunt: Aegithalos; Carduelis; Chloris; Coccothraustes; Erithacus; Galerida; Hippolais; Lullula; Oenanthe; Oriolus; Parus; Phoenicurus; Phylloscopus; Pinicola; Plectrophenax; Regulus; Remiz; Riparia; Saxicola; Sitta;

- C. long, narrow and pointed: Calandrella; Delichon; Fringilla; Luscinia; Passer; Prunella; Turdus;

- D. long and blunt: Acrocephalus; Anthus; Bombycilla; Cinclus; Emberiza; Eremophila; Loxia; Lusciniola; Lanius; Motacilla; Panurus; Serinus; Sturnus; Sylvia; Troglodytes;

\section{2. the cotyla dorsalis:}

- A. semicircular: Aegithalos; Anthus; Bombycilla; Calandrella; Carduelis; Certhia; Chloris; Cinclus; Erithacus; Fringilla; Hippolais; Lanius; Loxia; Lullula; Luscinia, Motacilla; Muscicapa, Parus; Passer; Phoenicurus; Phylloscopus; Plectrophenax; Prunella; Pyrrhula; Regulus; Remiz; Riparia; Sitta; Sturnus; Turdus;

- B. conical: Acrocephalus; Coccothraustes; Galerida; Eremophila; Hirundo; Locustella; Panurus; Serinus;

- C. elongated conical: Luscinola; Sylvia;

- D. truncated: Alauda; Emberiza; Oenanthe; Saxicola; Troglodytes; 
- E. asymmetrical: Delichon; Oriolus; Pinicola;

6.3. the tuberculum ligamentum collateralis ventralis:

- A. developed: Aegithalos; Alauda; Anthus; Bombycilla; Calandrella; Certhia; Cinclus; Delichon; Eremophila; Erithacus; Fringilla; Galerida; Hippolais; Hirundo; Loxia; Lullula; Luscinia; Lusciniola; Motacilla; Muscicapa; Oenanthe; Parus; Passer; Phoenicurus; Pinicola; Plectrophenax; Pyrrhula; Regulus; Remiz; Riparia; Saxicola; Sitta; Serinus; Sylvia; Turdus;

- B. undeveloped: Acrocephalus; Carduelis, Chloris; Coccothraustes; Emberiza; Lanius; Locustella; Oriolus; Panurus; Phylloscopus; Prunella; Sturnus; Troglodytes;

Alaudidae: Alauda ADA; Calandrella CAA; Eremophila DBA; Galerida BBA; Lullula BAA;

Hirundinidae: Delichon CEA; Hirundo ABA; Riparia BAA;

Paridae: Aegithalos BAA; Panurus DBB; Parus BAB; Remiz BAA;

Certhiidae: Certhia AAA;

Sittidae: Sitta BAA;

Muscicapidae: Erithacus BAA; Luscinia CAA; Muscicapa AAA; Oenanthe BDA; Phoenicurus BAA; Saxicola BDA;

Turdidae: Turdus CAA;

Oriolidae: Oriolus DEB;

Sylviidae: Acrocephalus DBB; Hippolais BAA; Locustella ABB; Lusciniola DCA; Phylloscopus BAB; Regulus BAA; Sylvia DCA;

Motacillidae: Anthus DAA; Motacilla DAA;

Bombycillidae: Bombycilla DAA;
Troglodytidae: Troglodytes DDB;

Cinclidae: Cinclus DAA;

Prunellidae: Prunella CAB;

Laniidae: Lanius DAB;

Sturnidae: Sturnus DAB;

Passeridae: Passer CAA;

Fringillidae: Carduelis BAB; Chloris BAB; Coccothraustes BBB; Fringilla CAA; Loxia DAA; Pinicola BEA; Pyrrhula AAA; Serinus DBA;

Emberizidae: Emberiza DDB; Plectrophenax BAA;

AAA: Certhia; Muscicapa; Pyrrhula;

ABA: Hirundo;

ABB: Locustella;

ABD: Monticola;

ADA: Alauda;

BAA: Aegithalos; Erithacus; Hippolais; Lullula; Parus; Phoenicurtus; Plectrophenax; Regulus; Remiz; Riparia; Sitta;

BAB: Carduelis; Chloris; Phylloscopus;

BBA: Galerida;

BBB: Coccothraustes;

BDA: Oenanthe; Saxicola;

BEA: Pinicola;

BEB: Oriolus;

CAA: Calandrella; Fringilla; Luscinia; Passer; Turdus;

CAB: Prunella;

CEA: Delichon;

DAA: Anthus; Bombycilla; Cinclus: Loxia; Motacilla;

DAB: Lanius; Sturnus;

DBA: Eremophila; Serinus;

DBB: Acrocephalus;

DBB: Panurus;

DCA: Lusciniola; Sylvia;

DDB: Emberiza; Troglodytes; 
7. Ulna (distal epiphysis) (Figure 10, Plate 25-28, Table 5)

\section{1. the shape of the condylus dorsalis:}

- A. pointed cone: Aegithalos; Calandrella; Carduelis; Delichon; Emberiza; Eremophila; Galerida; Loxia; Lusciniola; Phylloscopus; Regulus; Riparia; Sitta; Sylvia; Turdus;

- B. blunted cone: Acrocephalus; Alauda; Anthus; Chloris; Cinclus; Coccothraustes; Erithacus; Fringilla; Hippolais; Hirundo; Lanius; Locustella; Lullula; Luscinia; Motacilla, Parus; Phoenicurus; Pinicola; Plectrophenax; Prunella; Pyrrhula; Remiz; Saxicola; Serinus; Troglodytes;

- C. rounded: Bombycilla; Certhia; Muscicapa, Oenanthe; Oriolus; Panurus; Passer; Sturnus;

\section{2. the shape of the sulcus intercondy-} laris:

- A. curved: Acrocephalus; Anthus; Calandrella; Cinclus; Delichon; Emberiza; Eremophila; Erithacus; Fringilla; Galerida; Hippolais, Lanius; Locustella; Loxia; Lullula; Luscinia; Lusciniola; Oriolus; Parus; Phoenicurus; Phylloscopus, Plectrophenax; Prunella; Pyrrhula; Regulus; Remiz; Saxicola; Serinus; Sitta; Sylvia; Troglodytes; Turdus;

- B. asymmetrically curved: Aegithalos; Carduelis; Riparia;

- C. pointed: Alauda; Bombycilla; Certhia; Chloris; Coccothraustes; Hirundo; Motacilla; Muscicapa; Oenanthe; Panurus; Passer; Pinicola; Sturnus;

\section{3. the shape of the condylus ventralis:}

- A. conical: Calandrella; Delichon; Emberiza; Loxia; Riparia; Sylvia;

- B. blunt cone: Acrocephalus; Aegithalos; Anthus; Chloris; Cinclus; Eremophila;
Fringilla, Galerida; Hirundo; Locustella; Lullula; Lusciniola; Parus; Phoenicurus; Phylloscopus; Plectrophenax; Regulus; Remiz; Serinus; Sitta; Troglodytes;

- C. rounded: Alauda; Bombycilla; Carduelis; Certhia; Coccothraustes; Erithacus; Erithacus; Hippolais; Lanius; Luscinia; Motacilla; Muscicapa; Oenanthe; Oriolus; Panurus; Passer; Pinicola; Prunella; Pyrrhula, Saxicola; Sturnus; Turdus;

\section{4. the shape of the tuberculum carpale:}

- A. semicircular: Aegithalos; Alauda; Bombycilla, Calandrella; Certhia; Chloris; Coccothraustes; Emberiza; Eremophila; Erithacus; Fringilla, Galerida; Hippolais; Hirundo; Lanius; Loxia; Lullula; Luscinia; Lusciniola; Muscicapa, Panurus; Passer; Pinicola; Plectrophenax; Pyrrhula; Serinus; Sitta; Sturnus; Sylvia; Troglodytes; Turdus;

- B. conical: Phoenicurus; Phylloscopus;

- C. asymmetrical: Acrocephalus; Anthus; Carduelis; Delichon; Locustella; Motacilla; Oenenthe; Oriolus; Parus; Prunella; Remiz; Riparia; Saxicola;

- D. truncated: Cinclus; Regulus;

Alaudidae: Alauda BCCA; Calandrella AAAA; Eremophila AABA; Galerida AABA; Lullula BABA;

Hirundinidae: Delichon AAAC; Hirundo BCBA; Riparia ABAC;

Paridae: Aegithalos ABBA; Panurus CCCA; Parus BABC; Remiz BABC;

Certhiidae: Certhia CCCA; Sittidae: Sitta AABA;

Muscicapidae: Erithacus BACA; Luscinia BACA; Muscicapa CCCA; Oenanthe CCCC; Phoenicurus BABB; Saxicola BACC;

Turdidae: Turdus AACA; 
Oriolidae: Oriolus CACC;

Sylviidae: Acrocephalus BABC; Hippolais BACA; Locustella BABC; Lusciniola AABA; Phylloscopus AABB; Regulus AABD; Sylvia AAAA;

Motacillidae: Anthus BABC; Motacilla $\mathrm{BCCC}$;

Bombycillidae: Bombycilla CCCA;

Troglodytidae: Troglodytes BABA;

Cinclidae: Cinclus BABD;

Prunellidae: Prunella BACC;

Laniidae: Lanius BACA;

Sturnidae: Sturnus CCCA;

Passeridae: Passer CCCA;

Fringillidae: Carduelis ABCC; Chloris BCBA; Coccothraustes BCCA; Fringilla BABA; Loxia AAAA; Pinicola BCCA; Pyrrhula BACA; Serinus BABA;

Emberizidae: Emberiza AAAA; Plectrophenax BABA;

AAAA: Calandrella; Emberiza; Loxia; Sylvia;

AAAC: Delichon;

AABA: Eremophila; Galerida; Lusciniola; Sitta;

AABB: Phylloscopus;

AABD: Regulus;

AACA: Turdus;

ABAC: Riparia;

ABBA: Aegithalos;

ABCC: Carduelis;

ACBB: Erithacus;

BABA: Fringilla; Lullula; Plectrophenax; Serinus; Troglodytes;

BABB: Phoenicurus;

BABC: Acrocephalus; Anthus; Locustella; Parus; Remiz;

BABD: Cinclus;

BACA: Erithacus; Hippolais; Lanius; Luscinia; Luscinia; Pyrrhula;

BACC: Prunella; Saxicola;

BCBA: Chloris; Hirundo;
BCCA: Alauda; Coccothraustes; Pinicola; BCCC: Motacilla;

CACC: Oriolus;

CCCA: Bombycilla; Certhia; Muscicapa; Panurus; Passer; Sturnus;

CCCC: Oenanthe;

8. Carpometacarpus (Figure 11-12, Plate 29-32, Table 6)

\section{1. the shape of the a trochlea carpalis:}

- A. semicircle: Acrocephalus; Aegithalos; Anthus; Calandrella; Carduelis; Erithacus; Fringilla; Galerida; Hirundo; Loxia; Motacilla; Oenanthe; Oriolus; Parus; Passer; Phoenicurus; Plectrophenax; Regulus; Saxicola; Sylvia; Turdus;

- B. symmetrically cone: Delichon; Prunella; - C. symmetrically blunt cone: Alauda; Bombycilla; Chloris; Coccothraustes; Emberiza; Eremophila; Hippolais; Lanius; Locustella; Lullula; Luscinia; Monticola; Muscicapa; Pinicola; Pyrrhula; Riparia; Serinus; Sitta; Sturnus; Troglodytes;

- D. asymmetrically cone: Certhia; Cinclus; Panurus; Phylloscopus;

8.2. the form of the processus extensorius:

- A. straight pointy cone: Aegithalos; Hippolais; Luscinia; Parus; Regulus

- B. slanting pointy cone: Certhia; Cinclus; Coccothraustes; Hirundo;

- C. straight blunt cone: Acrocephalus; Alauda; Anthus; Emberiza; Erithacus; Loxia; Lullula; Motacilla; Muscicapa; Phylloscopus; Plectrophenax; Prunella; Pyrrhula; Saxicola;

- D. leaning blunt cone: Bombycilla; Calandrella; Cinclus; Delichon; Eremophila; Lanius; Locustella; Monticola; Oriolus; Passer; Pinicola; Serinus, Sitta; Sturnus; Troglodytes; Turdus; 
- E. rounded tips: Carduelis; Chloris; Emberiza; Fringilla; Loxia; Oenanthe; Panurus; Sylvia;

- F. asymmetrically: Galerida; Phoenicurus; Riparia;

\section{3. the shape of the processus alularis:}

- A. rectangular: Acrocephalus; Alauda; Anthus; Chloris; Erithacus; Hippolais; Hirundo; Lanius; Loxia; Lullula; Motacilla; Oenanthe; Phylloscopus; Plectrophenax; Saxicola; Serinus; Turdus;

- B. rounded rectangular: Calandrella; Carduelis; Delichon; Eremophila; Fringilla; Monticola; Panurus; Phoenicurus; Pinicola; Prunella; Sturnus;

- C. conical: Aegithalos; Bombycilla; Cinclus; Coccothraustes; Galerida; Luscinia; Oriolus; Parus; Passer; Pyrrhula; Riparia; Sitta; Troglodytes;

- D. irregular: Certhia; Emberiza; Locustella; Muscicapa; Regulus; Sylvia;

\section{4. the form of the fovea subalularis:}

- A. conical groove: Aegithalos; Alauda; Erithacus; Hippolais; Luscinia; Motacilla; Panurus; Passer; Phoenicurus; Phylloscopus; Pinicola; Prunella; Pyrrhula; Saxicola; Sylvia;

- B. irregular conical groove: Acrocephalus; Anthus; Calandrella; Galerida; Lullula; Plectrophenax;

- C. trapezoidal groove: Bombycilla; Certhia; Emberiza; Eremophila; Hirundo; Lanius; Monticola; Muscicapa; Sitta; Sturnus;

- D. irregular trapezoidal groove: Coccothraustes; Riparia; Serinus;

- E. semicircular groove: Cinclus; Delichon; Regulus; Turdus;

- F. miss: Carduelis; Chloris; Fringilla; Locustella; Loxia; Oenanthe; Oriolus; Parus; Troglodytes;
8.4. the character of the protuberantia metacarpale majus:

- A. advanced cone-shaped: Aegithalos; Bombycilla; Cinclus; Hirundo; Muscicapa; Phylloscopus; Pinicola; Pyrrhula; Regulus; Riparia; Sitta;

- B. advanced blunt cone-shaped: Certhia; Delichon; Monticola; Passer; Prunella;

- C. undeveloped conical: Acrocephalus; Alauda; Anthus; Calandrella; Carduelis; Chloris; Coccothraustes; Eremophila; Fringilla; Galerida; Hippolais; Loxia; Luscinia; Motacilla; Panurus; Parus; Phoenicurus; Saxicola; Serinus; Sturnus;

- D. undeveloped flattened: Emberiza; Erithacus; Locustella; Lullula; Oriolus; Plectrophenax; Sylvia; Turdus;

- E. miss: Lanius; Oenanthe; Troglodytes;

\section{5. the shape of the facies articularis di-} gitale minor:

- A. pointly concave: Acrocephalus; Alauda; Erithacus; Hippolais; Luscinia; Oenanthe; Panurus; Pinicola; Prunella; Sylvia; Turdus;

- B. rounded: Aegithalos; Carduelis; Certhia; Cinclus; Locustella; Loxia; Lullula; Passer; Phylloscopus; Plectrophenax;

- C. cut-off: Anthus; Chloris; Coccothraustes; Galerida; Motacilla; Oriolus; Parus; Pyrrhula; Riparia; Troglodytes;

- D. wavy: Bombycilla; Emberiza; Phoenicurus; Saxicola; Serinus;

- E. concave: Calandrella; Delichon; Eremophila; Fringilla; Hirundo; Lanius; Monticola; Muscicapa; Regulus; Sitta; Sturnus;

Alaudidae: Alauda CCAACA; Calandrella ADBBCE; Eremophila CDBCCE; Galerida AFCBCC; Lullula CCABDB;

Hirundinidae: Delichon BDBEBE; Hirundo ABACAE; Riparia CFCDAC; 
Paridae: Aegithalos AACAAB; Panurus DEBACA; Parus ADCFCC;

Certhiidae: Certhia DBDCBB;

Sittidae: Sitta CDCBAE;

Muscicapidae: Erithacus ACAADA; Luscinia CACACA Monticola CDBCBE; Muscicapa CCDCAE; Oenanthe AEAFEA; Phoenicurus AFBACD; Saxicola ACAACD;

Turdidae: Turdus ADAEDA;

Oriolidae: Oriolus ADCFDC;

Sylviidae: Acrocephalus ACABCA; Hippolais CAAACA; Locustella CDDFDB; Phylloscopus DCAAAB; Regulus AADEAE; Sylvia AEDADA;

Motacillidae: Anthus ACABCC; Motacilla ACAACC;

Bombycillidae: Bombycilla CDCCAD;

Troglodytidae: Troglodytes CDCFEC;

Cinclidae: Cinclus DBCEAB:

Prunellidae: Prunella BCBABA;

Laniidae: Lanius CDACEE;

Sturnidae: Sturnus CDBCCE;

Passeridae: Passer ADCABB;

Fringillidae: Carduelis AEBFCB; Chloris CFAFCC; Coccothraustes CBCDCC; Fringilla AEBFCE; Loxia ACAFCB; Pinicola CDBAAA; Pyrrhula CCCAAC; Serinus CDBDCD;

Emberizidae: Emberiza CCDCEDD; Plectrophenax ACABDB;

AACAAB: Aegithalos;

AADEAE: Regulus;

ABACAE: Hirundo;

ACAACC: Motacilla;

ACAACD: Saxicola;

ACAADA: Erithacus;

ACABCA: Acrocephalus;

ACABCC: Anthus;

ACABDB: Plectrophenax;

ACAFCB: Loxia;

ADAEDA: Turdus;

ADBBCE: Calandrella;
ADCABB: Passer;

ADCFCC: Parus;

ADCFDC: Oriolus;

AEAFEA: Oenanthe,

AEBFCB: Carduelis;

AEBFCE: Fringilla;

AEDADA: Sylvia;

AFBACD: Phoenicurus;

AFCBCC: Galerida;

BCBBBA: Prunella;

BDBEBE: Delichon;

CAAACA: Hippolais;

CACACA: Luscinia;

CBCDCC: Coccothraustes;

CCAACA: Alauda;

CCABDB: Lullula;

CCCAAC: Pyrrhula;

CCDCAE: Muscicapa;

CCDCED: Emberiza;

CDACEE: Lanius,

CDBAAA: Pinicola;

CDBCBE: Monticola;

CDBDCD: Serinus;

CDBCCE: Eremophila; Sturnus;

CDCBAE: Sitta;

CDCCAD: Bombycilla;

CDCFEC: Troglodytes;

CDDFDB: Locustella;

CEDAAB: Aegithalos;

CFAFCC: Chloris;

CFCDAC: Riparia;

DBCEAB: Cinclus;

DBDCBB: Certhia;

DCAAAB: Phylloscopus;

DEBACA: Panurus;

9. Phalanx proximalis digiti majoris (Figure 13, Plate 33-36, Table 7)

9.1. the form of the margo proximalis:

- A. strongly bulging: Acrocephalus; Aegithalos; Anthus; Calandrella; Carduelis; Certhia; Cettia; Coccothraustes; 
Eremophilla; Erithacus; Lanius; Loxia; Lullula; Motacilla; Muscicapa; Parus; Phylloscopus; Prunella; Sylvia;

- B. weakly bulging: Alauda; Bombycilla; Chloris; Delichon; Emberiza; Fringilla; Galerida; Hippolais; Hirundo; Monticola; Oenanthe; Oriolus; Phoenicurus; Pinicola; Pyrrhula; Regulus; Riparia; Saxicola; Serinus; Sitta; Sturnus; Turdus;

- C. concave: Locustella; Lusciniola; Troglodytes;

- D. wavy: Cinclus; Luscinia;

- E. flattened: Passer; Plectrophenax;

9.2. the form of the tuberculum ventralis:

- A. rounded: Alauda; Anthus; Certhia; Eremophilla; Erithacus; Fringilla; Hippolais; Lanius; Locustella; Loxia; Luscinia; Motacilla; Muscicapa; Parus; Phoenicurus; Phylloscopus; Pyrrhula; Regulus; Serinus; Sitta; Sturnus; Troglodytes; Turdus;

- B. flattened: Emberiza; Lullula; Plectrophenax;

- C. upwards protruding: Acrocephalus; Aegithalos; Bombycilla; Carduelis; Cettia; Chloris; Cinclus; Coccothraustes; Delichon; Galerida; Hirundo; Lusciniola; Monticola; Oriolus; Passer; Prunella; Riparia; Sylvia;

- D. conical: Calandrella; Oenanthe; Pinicola; Saxicola;

\section{3. the form of the tuberculum dorsalis:}

- A. conical: Acrocephalus; Aegithalos; Cettia; Cinclus; Chloris; Coccothraustes; Emberiza; Eremophilla; Erithacus; Hippolais; Locustella; Loxia; Lusciniola; Monticola; Muscicapa; Oenanthe; Oriolus; Parus; Passer; Phoenicurus; Pyrrhula; Regulus; Saxicola; Sitta; Troglodytes; Turdus;

- B. hook shaped: Calandrella; Lanius; Lullula; Phylloscopus; Sturnus; Sylvia;
- C. flattened: Alauda; Galerida; Prunella; Serinus;

- D. rounded: Anthus; Bombycilla; Carduelis; Certhia; Delichon; Fringilla; Hirundo; Luscinia; Motacilla; Plectrophenax; Pinicola; Riparia;

\section{4. the character of the margo dorsalis:}

- A. rounded: Eremophilla; Galerida; Hippolais; Lullula; Monticola; Oriolus; Pyrrhula;

- B. straight: Anthus; Lanius; Loxia; Muscicapa; Phylloscopus; Pinicola; Prunella; Sitta; Troglodytes;

- C. wavy: Acrocephalus; Aegithalos; Alauda; Bombycilla; Calandrella; Carduelis; Certhia; Cettia; Chloris; Cinclus; Coccothraustes; Delichon; Emberiza; Erithacus; Fringilla; Hirundo; Locustella; Luscinia; Lusciniola; Motacilla; Oenanthe; Parus; Passer; Phoenicurus; Plectrophenax; Regulus; Riparia; Saxicola; Serinus; Sturnus; Sylvia; Turdus;

\section{5. the character of the margo distalis:}

- A. rounded: Acrocephalus; Aegithalos; Alauda; Calandrella; Carduelis; Certhia; Cinclus; Eremophilla; Galerida; Locustella; Loxia; Lullula; Lusciniola; Motacilla; Oenanthe; Oriolus; Passer; Phoenicurus; Prunella; Pyrrhula; Saxicola; Sturnus; Sylvia;

- B. flattened: Chloris; Emberiza; Hippolais; Lanius; Monticola; Pinicola; Regulus;

- C. wavy: Anthus; Bombycilla; Cettia; Coccothraustes; Delichon; Erithacus; Fringilla; Hirundo; Luscinia; Muscicapa; Parus; Phylloscopus; Plectrophenax; Riparia; Serinus; Sitta; Troglodytes; Turdus;

Alaudidae: Alauda BACCA; Calandrella ADBCA; Eremophila AAAAA; Galerida BCCAA; Lullula ABBAA; 
Hirundinidae: Delichon BCDCC; Hirundo BCDCC; Riparia BCDCC;

Paridae: Aegithalos ACACA; Parus AAACC;

Certhiidae: Certhia AADCA;

Sittidae: Sitta BAABC;

Muscicapidae: Erithacus AAACC; Luscinia DADCC; Monticola BCAAB; Muscicapa AAABC; Oenanthe BDACA; Phoenicurus BAACA; Saxicola BDACA;

Turdidae: Turdus BAACC; Oriolidae: Oriolus BCAAA;

Sylviidae: Acrocephalus ACACA; Cettia ACACC; Hippolais BAAAB; Locustella CAACA; Lusciniola CCACA; Phylloscopus AABBC; Regulus BAACB; Sylvia ACBCA;

Motacillidae: Anthus AADBC; Motacilla AADCA;

Bombycillidae: Bombycilla BCDCC;

Troglodytidae: Troglodytes CAABC;

Cinclidae: Cinclus DCACA:

Prunellidae: Prunella ACCBA;

Laniidae: Lanius AABBB;

Sturnidae: Sturnus BABCA;

Passeridae: Passer ECACA;

Fringillidae: Carduelis ACDCA; Chloris BCACB; Coccothraustes ACACC; Fringilla BADCC; Loxia AAABA; Pinicola BDDBB; Pyrrhula BAAAA; Serinus BACCC;

Emberizidae: Emberiza BBACB; Plectrophenax EBDCC;

AAAAA: Eremophila;

AAABA: Loxia;

AAABC: Muscicapa;

AAACC: Erithacus; Parus;

AABBB: Lanius;

AABBC: Phylloscopus;

AADCA: Certhia; Motacilla;

AADBC: Anthus;

ABBAA: Lullula;
ACACA: Acrocephalus; Aegithalos;

ACACC: Cettia; Coccothraustes;

ACBCA: Sylvia;

ACCBA: Prunella;

ACDCA: Carduelis;

ADBCA: Calandrella;

BAAAA: Pyrrhula;

BAAAB: Hippolais;

BAABC: Sitta;

BAACA: Phoenicurus;

BAACB: Regulus;

BAACC: Turdus;

BABCA: Sturnus;

BACCA: Alauda;

BACCC: Serinus;

BADCC: Fringilla;

BBACB: Emberiza;

BCAAA: Oriolus;

BCAAB: Monticola;

BCACB: Chloris;

BCCAA: Galerida;

BCDCC: Bombycilla; Delichon; Hirundo; Riparia;

BDACA: Oenanthe; Saxicola;

BDDBB: Pinicola;

CAABC: Troglodytes;

CAACA: Locustella;

CCACA: Lusciniola;

DADCC: Luscinia;

DCACA: Cinclus;

EBDCC: Plectrophenax;

ECACA: Passer;

10. Femur (distal epiphysis) (Figure 14, Plate 37-40, Table 8)

10.1. the shape of the condylus medialis:

- A. semicircle: Aegithalos; Bombycilla; Calandrella; Carduelis; Certhia; Cettia; Cinclus; Coccothraustes; Delichon; Erithacus; Galerida; Hippolais; Hirundo; Locustella; Loxia; Lullula; Monticola; Motacilla; Oriolus; Parus; Passer; 
Phoenicurus; Phylloscopus; Pinicola; Regulus; Sitta; Sturnus; Troglodytes

- B. blunt cone: Alauda; Anthus; Chloris; Emberiza; Eremophila; Fringilla; Lanius; Luscinia; Lusciniola; Oenanthe; Plectrophenax; Prunella; Pyrrhula; Remiz; Riparia; Saxicola; Serinus; Sylvia; Turdus; - C. asymmetrically: Acrocephalus;

\section{2. the character of the sulcus intercon-} dylaris:

- A. deeply concave: Acrocephalus; Aegithalos; Alauda; Calandrella; Carduelis; Cettia; Chloris; Cinclus; Eremophila; Erithacus; Fringilla; Lanius; Locustella; Lullula; Monticola; Motacilla; Oenanthe; Oriolus; Phoenicurus; Phylloscopus; Pinicola; Pyrrhula; Saxicola; Sturnus; Troglodytes;

- B. weakly concave: Certhia; Delichon; Emberiza; Galerida; Hippolais; Hirundo; Loxia; Lusciniola; Parus; Prunella; Regulus; Remiz; Serinus; Sylvia;

- C. asymmetrically concave: Anthus; Luscinia; Passer; Riparia;

- D. flattened: Coccothraustes; Plectrophenax; Sitta; Turdus;

- E. wavy: Bombycilla;

\section{3. the shape of the condylus lateralis:}

- A. broadly rounded: Acrocephalus; Anthus; Certhia; Cettia; Chloris; Eremophila; Delichon; Galerida; Hippolais; Hirundo; Oenanthe; Oriolus; Passer; Phylloscopus; Prunella; Pyrrhula; Remiz; Sturnus; Sylvia; Turdus;

- B. rounded: Aegithalos; Alauda; Bombycilla; Carduelis; Cinclus; Coccothraustes; Erithacus; Lanius; Loxia; Lullula; Luscinia; Monticola; Motacilla; Parus; Phoenicurus; Pinicola; Regulus; Serinus; Sitta; Troglodytes;
- C. conical: Calandrella; Emberiza; Fringilla; Locustella; Lusciniola; Plectrophenax; Riparia; Saxicola;

\section{4. the form of the epicondylus medialis:}

- A. pointedly protruding: Acrocephalus; Calandrella; Coccothraustes; Delichon; Fringilla; Hippolais; Parus; Phylloscopus; Pinicola; Serinus; Sturnus;

- B. rounded protruding: Cettia; Emberiza; Eremophila; Erithacus; Galerida; Locustella; Luscinia; Monticola; Passer; Pyrrhula; Regulus; Saxicola; Troglodytes; - C. bulging: Aegithalos; Alauda; Anthus; Bombycilla; Carduelis; Certhia; Cinclus; Loxia; Lullula; Motacilla; Oriolus; Phoenicurus; Plectrophenax; Remiz; Sitta;

- D. not arching: Chloris; Hirundo; Lanius; Lusciniola; Oenanthe; Prunella; Riparia; Sylvia; Turdus;

\section{5. the form of the epicondylus lateralis:}

- A. conical protruding: Acrocephalus; Emberiza; Fringilla; Passer;

- B. rounded protruding: Alauda; Calandrella; Carduelis; Loxia; Lullula; Parus; Phylloscopus; Pinicola; Plectrophenax; Prunella; Pyrrhula; Serinus;

- C. bulging: Aegithalos; Anthus; Bombycilla; Chloris; Cinclus; Coccothraustes; Delichon; Eremophila; Erithacus; Hippolais; Hirundo; Lanius; Locustella; Loxia; Luscinia; Lusciniola; Monticola; Motacilla; Oenanthe; Regulus; Remiz; Riparia; Sylvia; Sitta; Sturnus; Troglodytes; Turdus;

- D. not arching: Certhia; Cettia; Galerida; Oriolus; Phoenicurus; Saxicola;

Alaudidae: Alauda BABCB; Calandrella AACAB; Eremophila BAABC; Galerida ABABD; Lullula AABCB;

Hirundinidae: Delichon ABAAC; Hirundo ADADC; Riparia BCCDC; 
Paridae: Aegithalos AABCC; Parus ABBAB; Remiz BBACC;

Certhiidae: Certhia ABACD;

Sittidae: Sitta ADBCC;

Muscicapidae: Erithacus AABBC; Luscinia DABBC; Monticola ACBBC; Oenanthe BAADC; Phoenicurus AABCD; Saxicola BBABD;

Turdidae: Turdus BDADC;

Oriolidae: Oriolus AAACD;

Sylviidae: Acrocephalus CAAAA; Cettia AAABD; Hippolais ABAAC; Locustella AACBC; Lusciniola BBCDC; Phylloscopus AAAAB; Regulus ABBBC; Sylvia ACBDC;

Motacillidae: Anthus BCACC; Motacilla AABCC;

Bombycillidae: Bombycilla AEBCC; Troglodytidae: Troglodytes AABBC;

Cinclidae: Cinclus AABCC;

Prunellidae: Prunella BBADB;

Laniidae: Lanius BABDC;

Sturnidae: Sturnus AAAAC;

Passeridae: Passer ACABA;

Fringillidae: Carduelis AABCB; Chloris BAADC; Coccothraustes ADBAC; Fringilla BACAA; Loxia ABBCC; Pinicola AABAB; Pyrrhula BAABB; Serinus BBBAB;

Emberizidae: Emberiza BBCBA; Plectrophenax BDCCB;

AAAAB: Phylloscopus;

AAAAC: Sturnus;

AAABD: Cettia;

AAACD: Oriolus;

AABAB: Pinicola;

AABBC: Erithacus; Troglodytes;

AABCB: Carduelis; Lullula;

AABCC: Aegithalos; Cinclus: Motacilla;

AABCD: Phoenicurus;

AACAB: Calandrella;

AACBC: Locustella;

ABAAC: Delichon; Hippolais;
ABABD: Galerida;

ABACD: Certhia;

ABBAB: Parus;

ABBCC: Loxia;

ABBDC: Regulus;

ACABA: Passer;

ACBBC: Monticola;

ACBDC: Sylvia;

ADADC: Hirundo;

ADBAC: Coccothraustes;

ADBCC: Sitta;

AEBCC: Bombycilla;

BAABB: Pyrrhula;

BAABC: Eremophila;

BAADC: Chloris; Oenanthe;

BACAA: Fringilla;

BABCB: Alauda;

BABCC: Remiz;

BABDC: Lanius;

BBABD: Prunella; Saxicola;

BBBAB: Serinus;

BBCBA: Emberiza;

BBCDC: Lusciniola;

BCACC: Anthus;

BCCDC: Riparia;

BDADC: Turdus;

BDCCB: Plectrophenax;

CAAAA: Acrocephalus;

DABBC: Luscinia;

11. Tibiotarsus (distal epiphysis) (Figure 15, Plate 41-44, Table 9)

11.1. the shape of the epicondylus medialis: - A. blunt cone: Alauda; Acrocephalus; Aegithalos; Bombycilla; Calandrella; Certhia; Coccothraustes; Emberiza; Eremophila; Erithacus; Fringilla; Hippolais; Lanius; Loxia; Luscinia; Monticola; Motacilla; Muscicapa; Oenanthe; Oriolus; Parus; Passer; Phoenicurus; Pinicola; Plectrophenax; Pyrrhula; Remiz; Saxicola; Serinus; Sitta; Sturnus; Troglodytes; Turdus; 
- B. conical: Cinclus; Locustella; Lusciniola; Prunella;

- C. rounded: Anthus; Carduelis; Delichon; Galerida; Hirundo; Lullula; Melanocorypha; Phylloscopus; Regulus; Riparia; Sylvia;

\section{2. the shape of the epicondylus lateralis:}

- A. rounded; Alauda; Cinclus; Erithacus; Hirundo; Lullula; Luscinia; Monticola; Parus; Phylloscopus; Riparia; Saxicola; Sturnus; Troglodytes;

- B. blunt cone: Aegithalos; Bombycilla; Calandrella; Carduelis; Coccothraustes; Delichon; Eremophila; Fringilla; Galerida; Hippolais; Lanius; Loxia; Lusciniola; Motacilla; Muscicapa; Oriolus; Passer; Phoenicurus; Pinicola; Prunella; Pyrrhula; Regulus; Remiz; Serinus; Sitta; Sylvia; Turdus;

- C. conical: Acrocephalus; Anthus; Emberiza; Locustella; Melanocorypha; Oenanthe; Plectrophenax;

- D. hook shape: Certhia;

11.3. the form of the incisura intercondylaris:

- A. wavy: Acrocephalus; Aegithalos; Coccothraustes; Delichon; Fringilla; Hippolais; Lanius; Loxia; Lusciniola; Motacilla; Muscicapa; Oriolus; Parus; Passer; Pinicola; Pyrrhula; Regulus; Remiz; Serinus; Sitta; Sturnus; Sylvia; Troglodytes;

- B. deeply arched: Alauda; Bombycilla; Calandrella; Carduelis; Eremophila; Galerida; Melanocorypha;

- C. asymmetrically arched: Phoenicurus; Prunella; Turdus; Locustella; Phylloscopus; Riparia;

- D. asymmetrically wavy: Anthus; Certhia; Emberiza; Erithacus; Lullula; Luscinia; Monticola; Oenanthe; Plectrophenax; Saxicola;

- E. flattened: Cinclus; Hirundo;

\section{4. the character of the tuberositas re-} tinaculi:

- A. below pointly protruding: Acrocephalus; Anthus; Locustella; Melanocorypha; Motacilla; Phylloscopus; Sylvia;

- B. below rounded protruding: Certhia; Cinclus; Erithacus; Galerida; Hippolais; Loxia; Luscinia; Lusciniola; Monticola; Oenanthe; Plectrophenax; Prunella; Pyrrhula; Remiz; Sitta;

- C. below weakly rounded: Alauda; $\mathrm{Ca}$ landrella; Delichon; Fringilla; Passer; Pinicola; Serinus;

- D. high pointly protruding: Emberiza; Lullula; Saxicola; Turdus;

- E. high rounded protruding: Aegithalos; Bombycilla; Carduelis; Coccothraustes; Eremophila; Lanius; Muscicapa; Parus; Phoenicurus; Regulus; Remiz; Riparia; Sturnus; Troglodytes;

- F. miss: Hirundo; Oriolus;

Alaudidae: Alauda AABC; Calandrella ABBC; Eremophila ABBE; Galerida CBBB; Lullula CADD; Melanocorypha CCBA;

Hirundinidae: Delichon CBAC; Hirundo CAEF; Riparia CACE;

Paridae: Aegithalos ABAE; Parus AAAE; Remiz ABAE;

Certhiidae: Certhia ADDB;

Sittidae: Sitta ABAB;

Muscicapidae: Erithacus AADB; Luscinia AADB; Monticola AADB; Muscicapa ABAE; Oenanthe AADB; Phoenicurus ABCE; Saxicola AADD;

Turdidae: Turdus ABCD;

Oriolidae: Oriolus ABAF;

Sylviidae: Acrocephalus ACAA; Hippolais ABAB; Locustella BCCA; Lusciniola BBAB; Phylloscopus CACA; Regulus CBAE; Sylvia CBAA;

Motacillidae: Anthus CCDA; Motacilla ABAA; 
Bombycillidae: Bombycilla ABBE;

Troglodytidae: Troglodytes AAAE;

Cinclidae: Cinclus BAEB;

Prunellidae: Prunella BBCB;

Laniidae: Lanius ABAE;

Sturnidae: Sturnus AAAE;

Passeridae: Passer BBAC;

Fringillidae: Carduelis CBBE; Coccothraustes ABAE; Fringilla ABAC; Loxia ABAB; Pinicola ABAC; Pyrrhula ABAB; Serinus ABAC;

Emberizidae: Emberiza ACDD; Plectrophenax ACDB;

AAAE: Parus; Sturnus; Troglodytes;

AABC: Alauda;

AADB: Erithacus; Luscinia; Monticola; Oenanthe;

AADD: Saxicola;

ABAA: Motacilla;

ABAB: Hippolais; Loxia; Pyrrhula; Sitta;

ABAC: Fringilla; Pinicola; Serinus;

ABAE: Aegithalos; Coccothraustes; Lanius; Muscicapa; Remiz;

ABAF: Oriolus;

ABBC: Calandrella;

ABBE: Bombycilla; Eremophila;

ABCD: Turdus;

ABCE: Phoenicurus;

ACAA: Acrocephalus;

ACDB: Plectrophenax;

ACDD: Emberiza;

ADDB: Certhia;

BAEB: Cinclus;

BBAC: Passer;

BBAB: Lusciniola;

BBCB: Prunella;

BCCA: Locustella;

CACA: Phylloscopus;

CACE: Riparia;

CADD: Lullula;

CAEF: Hirundo;

CBAA: Sylvia;
CBAC: Delichon;

CBAE: Regulus;

CBBA: Melanocorypha;

CBBB: Galerida;

CBBE: Carduelis;

CCDA: Anthus;

12. Tarsometatarsus (distal epiphysis)

(Figure 16, Plate 45-48, Table 10)

12.1. the character of the margo medialis:

- A. straight: Anthus; Calandrella; Delichon; Emberiza; Erithacus; Hippolais; Hirundo; Lanius; Loxia; Luscinia; Monticola; Motacilla; Oenanthe; Oriolus; Passer; Pinicola; Prunella; Regulus; Saxicola;

- B. wavy: Alauda; Bombycilla; Carduelis; Cettia; Chloris; Coccothraustes; Eremophila; Fringilla; Galerida; Locustella; Lullula; Lusciniola; Melanocorypha; Muscicapa; Phoenicurus; Plectrophenax; Pyrrhula; Remiz; Riparia; Sylvia; Troglodytes; Turdus;

- C. bulging: Certhia; Sitta;

- D. concave: Acrocephalus; Aegithalos; Parus; Phylloscopus; Sturnus;

12.2. the shape of the troclea metatarsi II.:

- A. with cut-off end: Anthus; Carduelis; Chloris; Coccothraustes; Delichon; Fringilla; alerida; Hirundo; Lullula; Melanocorypha; Monticola; Motacilla; Passer; Phoenicurus; Plectrophenax; Prunella; Riparia; Sturnus; Turdus;

- B. with rounded end: Acrocephalus; Aegithalos; Alauda; Bombycilla; Calandrella; Emberiza; Eremophila; Lusciniola; Muscicapa; Oenanthe; Oriolus; Regulus; Remiz; Saxicola; Sitta; Troglodytes;

- C. asymmetrically: Certhia; Cettia; Locustella; Loxia; Luscinia; Parus; Phylloscopus; Pyrrhula; 
- D. conical: Erithacus; Hippolais; Lanius; Pinicola; Sylvia;

12.3. the shape of the trochlea metatarsi III.: - A. weakly concave: Aegithalos; Alauda; Anthus; Emberiza; Eremophila; Fringilla; Hippolais; Hirundo; Lanius; Locustella; Lusciniola; Motacilla; Oriolus; Pinicola; Plectrophenax; Prunella; Pyrrhula; Sylvia;

- B. concave: Acrocephalus; Bombycilla; Calandrella; Carduelis; Cettia; Chloris; Coccothraustes; Delichon; Erithacus; Galerida; Loxia; Lullula; Luscinia; Melanocorypha; Monticola; Muscicapa; Oenanthe; Parus; Passer; Phoenicurus; Phylloscopus; Regulus; Remiz; Riparia; Saxicola; Sitta; Sturnus; Troglodytes; Turdus;

- C. strongly concave: Certhia;

12.4. the shape of the trochlea metatarsi IV.:

- A. conical: Cettia; Chloris; Eremophila; Melanocorypha; Monticola; Motacilla; Muscicapa; Phoenicurus; Prunella; Sturnus; Turdus;

- B. rounded blunt cone: Alauda; Bombycilla; Carduelis; Certhia; Delichon; Erithacus; Fringilla; Hirundo; Locustella; Oenanthe; Parus; Passer; Phylloscopus; Pinicola; Plectrophenax; Pyrrhula; Remiz; Remiz; Riparia; Saxicola; Sylvia; Troglodytes;

- C. cut-off: Acrocephalus; Aegithalos; $\mathrm{Ca}$ landrella; Coccothraustes; Emberiza; Lanius; Loxia; Lullula; Luscinia; Lusciniola; Regulus;

- D. asymmetrically: Anthus; Hippolais; Galerida; Oriolus; Sitta;

\section{5. the form of the incisura intertrochle- aris medialis:}

- A. narow: Aegithalos; Alauda; Calandrella; Carduelis; Coccothraustes; Delichon; Emberiza; Eremophila; Fringilla;
Hirundo; Lanius; Locustella; Loxia; Lusciniola; Monticola; Oenanthe; Oriolus; Parus; Passer; Pinicola; Prunella; Riparia; Sitta; Sturnus; Sylvia; Troglodytes;

- B. wide: Acrocephalus; Anthus; Bombycilla; Certhia; Cettia; Chloris; Erithacus; Galerida; Hippolais; Lullula; Luscinia; Melanocorypha; Motacilla; Muscicapa; Phoenicurus; Phylloscopus; Plectrophenax; Pyrrhula; Regulus; Remiz; Saxicola; Turdus;

\section{6. the form of the incisura intertrochle-} aris lateralis:

- A. narow: Aegithalos; Alauda; Calandrella; Carduelis; Chloris; Coccothraustes; Delichon; Fringilla; Hirundo; Lullula; Locustella; Luscinia; Lusciniola; Parus; Passer; Phylloscopus; Regulus; Riparia; Sitta; Sylvia; Troglodytes;

- B. wide: Acrocephalus; Anthus; Bombycilla; Certhia; Cettia; Emberiza; Eremophila; Erithacus; Galerida; Hippolais; Lanius; Loxia; Melanocorypha; Monticola; Motacilla; Muscicapa; Oenanthe; Oriolus; Phoenicurus; Pinicola; Plectrophenax; Prunella; Pyrrhula; Remiz; Saxicola; Sturnus; Turdus;

Alaudidae: Alauda BBABAA; Calandrella ABBCAA; Eremophila BBAAAB; Galerida BABDBB; Lullula BABBBA; Melanocorypha $\mathrm{BABABB}$;

Hirundinidae: Delichon AABBAA; $\mathrm{Hi}^{-}$ rundo AAABAA; Riparia BABBAA;

Paridae: Aegithalos DBACAA; Parus DCBBAA; Remiz BBBBBB;

Certhiidae: Certhia CCCBBB;

Sittidae: Sitta CBBDAA;

Muscicapidae: Erithacus ADBBAB; Luscinia ACBCBA; Monticola AABAAB; Muscicapa BBBABB; Oenanthe ABBBAB; Phoenicurus BBBABB; Saxicola ABBBBB; 
Turdidae: Turdus BABABB;

Oriolidae: Oriolus ABADAB;

Sylviidae: Acrocephalus DBBCBB; Cettia BCBABB; Hippolais ACADBB; Locustella BCABAA; Lusciniola BBACAA; Phylloscopus DCBBBA; Regulus ABBCBA; Sylvia BDABAA;

Motacillidae: Anthus ABADBB; Motacilla ABAABB;

Bombycillidae: Bombycilla BBBBBB;

Troglodytidae: Troglodytes BBBBAA;

Prunellidae: Prunella AAAAAB;

Laniidae: Lanius ABACBB;

Sturnidae: Sturnus DABAAB;

Passeridae: Passer ADBBAA;

Fringillidae: Carduelis BABBAA; Chloris BABABA; Coccothraustes BABCAA; Fringilla BAABAA; Loxia ACBCBB; Pinicola ABABAB; Pyrrhula $\mathrm{BCABBB}$;

Emberizidae: Emberiza ABACBB; Plectrophenax BAABBB;

AAAAAB: Prunella;

AAABAA: Hirundo;

AABAAB: Monticola;

AABBAA: Delichon;

ABAABB: Motacilla;

ABACBB: Emberiza; Lanius;

ABADAB: Oriolus;

ABADBB: Anthus;

ABBBAB: Oenanthe;

ABBBBB: Saxicola;

ABBCAA: Calandrella;

ABBCBA: Regulus;

ACADBB: Hippolais;

ACBCBA: Luscinia;

ACBCBB: Loxia;

ADABAB: Pinicola;

ADBBAA: Passer;

ADBBAB: Erithacus;

BAABAA: Carduelis; Fringilla;

BAABBB: Plectrophenax;

BABABA: Chloris;
BABABB: Melanocorypha; Turdus;

BABBAA: Carduelis; Riparia;

BABBBA: Lullula;

BABCAA: Coccothraustes;

BABDBB: Galerida;

BBAAAB: Eremophila;

BBABAA: Alauda;

BBACAA: Lusciniola;

BBBABB: Muscicapa; Phoenicurus;

BBBBAA: Troglodytes;

BBBBBB: Bombycilla; Remiz;

BCABAA: Locustella;

BCABBB: Pyrrhula;

BCBABB: Cettia;

BDABAA: Sylvia;

CBBDAA: Sitta;

CCCBBB: Certhia;

DABAAB: Sturnus;

DBACAA: Aegithalos;

DBBCBB: Acrocephalus;

DCBBAA: Parus;

DCBBBA: Phylloscopus;

13. Phalanx ungularis (Figure 17, Plate 49-52, Table 11)

13.1. the direction and the form of the $t u$ berculum extensorium:

- A. straight and pointed: Anthus; Coccothraustes; Monticola; Passer; Prunella; Pyrrhula; Riparia; Sturnus; Turdus;

- B. straight and blunt: Aegithalos; Acrocephalus; Emberiza; Eremophila; Oriolus; Phoenicurus; Phylloscopus; Pinicola; Regulus; Saxicola; Serinus;

- C. dorsal rises and pointed: Certhia; Fringilla; Galerida; Muscicapa; Sitta;

- D. dorsal rises and blunted: Alauda; Hippolais; Hirundo; Luscinia; Oenanthe; Remiz;

- E. ventral available: Bombycilla; Lanius; Loxia; 
13.2. the character of the cotyla articularis:

- A. weakly concave: Acrocephalus; Aegithalos; Alauda; Bombycilla; Coccothraustes; Emberiza; Fringilla; Hippolais; Lanius; Luscinia; Monticola; Muscicapa; Oenanthe; Oriolus; Phoenicurus; Phylloscopus; Pinicola; Pyrrhula; Remiz; Riparia; Saxicola; Serinus; Sitta; Sturnus; Turdus;

- B. strongly concave: Anthus; Certhia; Eremophila; Galerida; Hirundo; Loxia; Passer; Prunella; Regulus;

13.3. the shape of the tuberculum flexorium:

- A. flattened: Acrocephalus; Anthus; Bombycilla, Hirundo; Loxia; Monticola; Riparia; Serinus; Sturnus;

- B. concave: Aegithalos; Emberiza; Fringilla; Muscicapa; Oenanthe; Phoenicurus; Prunella; Pyrrhula; Remiz; Saxicola;

- C. pointedly protruding: Eremophila; Oriolus;

- D. dully protruding: Alauda; Certhia; Coccothraustes; Galerida; Luscinia; Passer; Pinicola; Sitta; Turdus;

- E. wavy: Hippolais; Lanius; Phylloscopus; Regulus;

\section{4. the elbow of the margo plantaris:}

- A. weakly arched: Alauda; Eremophila; Galerida: Loxia; Pinicola; Prunella;

- B. symmetrically strongly arched: Acrocephalus; Aegithalos; Bombycilla; Certhia; Emberiza; Hippolais; Hirundo; Luscinia; Monticola; Muscicapa; Oenanthe; Phoenicurus; Phylloscopus; Pyrrhula; Remiz; Saxicola; Serinus; Sitta;

- C. asymmetrically strongly arched: Anthus; Coccothraustes; Fringilla; Lanius; Oriolus; Passer; Regulus; Riparia; Sturnus; Turdus;

\section{5. the form of the apex:}

- A. narow: Certhia; Coccothraustes; Emberiza; Fringilla; Hirundo; Muscicapa; Oenanthe; Passer; Phoenicurus; Phylloscopus; Riparia; Saxicola; Sitta; Turdus;

- B. stout: Acrocephalus; Aegithalos; Alauda; Anthus; Bombycilla; Eremophila; Galerida: Hippolais; Lanius; Loxia; Luscinia; Monticola; Oriolus; Pinicola; Prunella; Pyrrhula; Regulus; Remiz; Serinus; Sturnus;

Alaudidae: Alauda DADAB; Eremophila BBCAB; Galerida CBDAB;

Hirundinidae: Hirundo DBABA; Riparia AAACA;

Paridae: Aegithalos BABBB; Remiz DABBB;

Certhiidae: Certhia CBDBA;

Sittidae: Sitta CADBA;

Muscicapidae: Luscinia DADBB; Monticola AAABB; Muscicapa CABBA; Oenanthe DABBA; Phoenicurus BABBA; Saxicola BABBA;

Turdidae: Turdus AADCA;

Oriolidae: Oriolus BACCB;

Sylviidae: Acrocephalus BAABB; Hippolais DAEBB; Phylloscopus BAEBA; Regulus BBECB;

Motacillidae: Anthus ABACB;

Bombycillidae: Bombycilla EAABB;

Prunellidae: Prunella ABBAB;

Laniidae: Lanius EAECB;

Sturnidae: Sturnus AAACB;

Passeridae: Passer ABDCA;

Fringillidae: Coccothraustes AADCA; Fringilla CABCA; Loxia EBAAB; Pinicola BADAB; Pyrrhula AABBB; Serinus BAABB;

Emberizidae: Emberiza BABBA; 
AAABB: Pyrrhula;

AAACB: Sturnus;

AAACA: Riparia;

AAABB: Monticola;

AADCA: Coccothraustes; Turdus;

ABACB: Anthus;

ABBAB: Prunella;

ABDCA: Passer;

BAABB: Acrocephalus; Serinus;

BABBA: Emberiza; Phoenicurus; Saxicola;

BABBB: Aegithalos;

BACCB: Oriolus;

BAEBA: Phylloscopus;

BBCAB: Eremophila;

BADAB: Pinicola;

BBECB: Regulus;

CABBA: Muscicapa;

CABCA: Fringilla;

CADBA: Sitta;

CBDAB: Galerida;

CBDBA: Certhia;
DABBA: Oenanthe;

DABBB: Remiz;

DADAB: Alauda;

DADBB: Luscinia;

DAEBB: Hippolais;

DBABA: Hirundo;

EAABB: Bombycilla;

EAECB: Lanius;

EBAAB: Loxia;

\section{Acknowledgements}

The author wishes to express his gratitude to Mihály Gasparik for acces to recent bird bone collection in the Natural History Museum of Hungary Budapest, to Béla Solti, Tibor Pecsics and Péter Ujhelyi for their help in the revision of the text and to József Vuts and Lóránd Abos for the language revision. 


\section{References}

Ballmann, P. 1966. Die Vögel aus der altburdigalen Spaltenfüllung von Wintershof (West) bei Eichstätt [The birds of the altburdigalen fissure filling of Wintershof (West) in Eichstaett]. - Inaugural Dissertation zur erlangung der Doktorwürde der nHohe Naturwissenschaftlichen Fakultät der Ludwig Maximiliane, Universität zu München, München, pp. 115 (in German)

Baumel, J. J., King, A. S., Lucas, A. M., Breazile, J. E. \& Evans, H. E. 1979. Nomina anatomica avium. An annotated anatomical dictionary of birds. - Academical Press, London, pp. 637

Cheneval, J. 1983. Révision de l'avifaune du gisement aquitanien de Saint-Gérand-le-Puy (Allier, France) (Gaviiformes à Anseriformes) [Review of the avifauna of aquitanien deposit of Saint-Gérand-le-Puy (Allier, France) (Gaviiformes to Anseriformes)]. - Unpublished Thesis (Nr. 1380) Université Claude-Bernard, Lyon, pp. 220 (in French)

Cramp, S. (ed.) 1998. The Complete Birds of the Western Palearctic on CD rom. - Oxford University Press, UK

Cuisin, J. 1989. L'identification des crânes de passereaux (Passeriformes: Aves) [The identification of skulls passerines (Passeriformes, Aves)]. Doct. Dissertation, Universite de Bourgogne, pp. 340 (in French)

Driesch, A. von den 1976. A guide to the measurements of animal bones from archaeological sites. - Peabody Museum Bulletin 1., pp. 148

Fürbringer, M. 1888. Untersuchungen zur Morphologie und Systematik der Vögel, zugleich ein Beitrag zur Anatomie der Stutz- und Bewegungeorgane [Studies on the morphology and systematics of birds, also contribute to the anatomy of the mounting socket and locomotor organe]. - Amsterdam, Jena, pp. 421 (in German)

Gál, E. 2002. Avifauna pleistocena a Romaniei [Pleistocene bird faunas of Romania]. - Unpublied C.Sc Dissertation, Universitatea din București, Facultatea de Geologie București, pp. 263 (in Romanian)

Gilbert, B. M., Martin, L. D. \& Savage, H. G. 1981. Avian Osteology. - Library of Congress, Wyoming, pp. 252

Jánossy, D. 1983. Humeri of central European smaller Passeriformes. - Fragmenta Mineralogica et Paleontologica 11: 85-112.

Kessler, E. 2013a A Kárpát-medence madárvilágának öslénytani kézikönyve [Paleontological handbook of birdlife in the Carpathian Basin]. Könyvmühely, Miskolc, pp. 508 (in Hungarian)
Kessler, E. 2013b Neogene songbirds (Aves, Passeriformes) faunae from Hungary. - Hantkeniana 2013: 37-149.

Kessler, E. \& Moldvai, M. 1993. Studii biometrice şi morfologice asupra scheletului membrelor la Corvidae (Aves) (Partea I.) [Biometrical and morphological studies of limbs scheleton to Corvidae (Aves) (Part I.)]. - Studia Universitatis Babes-Bolyai, Series Biologia 38: 69-80 (in Romanian with English Summary)

Lambrecht, K. 1933. Handbuch der Palaeornithologie [Handbook of Palaeornithology]. - Gebrüder Borntraeger Berlin, pp. 1024 (in German)

Milne-Edwards, A. 1867-1868. Recherches anatomiques et paléontologiques pour servir à l'histoire des oiseaux fossiles de la France [Anatomical and paleontological research to serve a history the fossil birds of France]. Vol. 1. - Victor Masson et Fils, Paris, pp. 472+ pls 96 (in French)

Moreno, E. 1985. Clave osteologica para la identification de los Passeriformes Ibericos, I. [Osteological identification key for the Iberian Passerines I.]. Ardeola 32(2): 295-377. (in Spanish)

Moreno, E. 1986. Clave osteologica para la identification de los Passeriformes Ibericos, II. [Osteological identification key for the Iberian Passerines II.]. - Ardeola 33(1-2): 69-129. (in Spanish)

Moreno, E. 1987. Clave osteologica para la identification de los Passeriformes Ibericos, III. [Osteological identification key for the Iberian Passerines III.]. - Ardeola 34(2): 243-273. (in Spanish)

Mourer-Chauviré, C. 1975. Faunes d'oiseaux du Pléistocène de France: systématique, évolution et adaptation, interprétation paléoclimatique [Bird Fauna of the Pleistocene of France: systematics, evolution and adaptation, paleoclimatic interpretation]. - Géobios 8: 333-352. (in French with English Summary)

Solti, B. 1996. The comparative osteomorphological study of the European small-statured Falcons (Aves: Falconidae). - Folia Historico Naturalia Musei Matraensis 21: 5-282.

Tomek, T. \& Bochenṡki, Z. M. 2000. The comparative osteology of European corvids (Aves: Corvidae), with a key to the identification of their skeletal elements. - Publications of the Institute of Systematics and Evolution of Animals, Kraków, pp. 102

Ujhelyi, P. 1992. Identification of European finch species (Fringillidae) based on skull morphology keys. - Aquila 99: 99-110. 


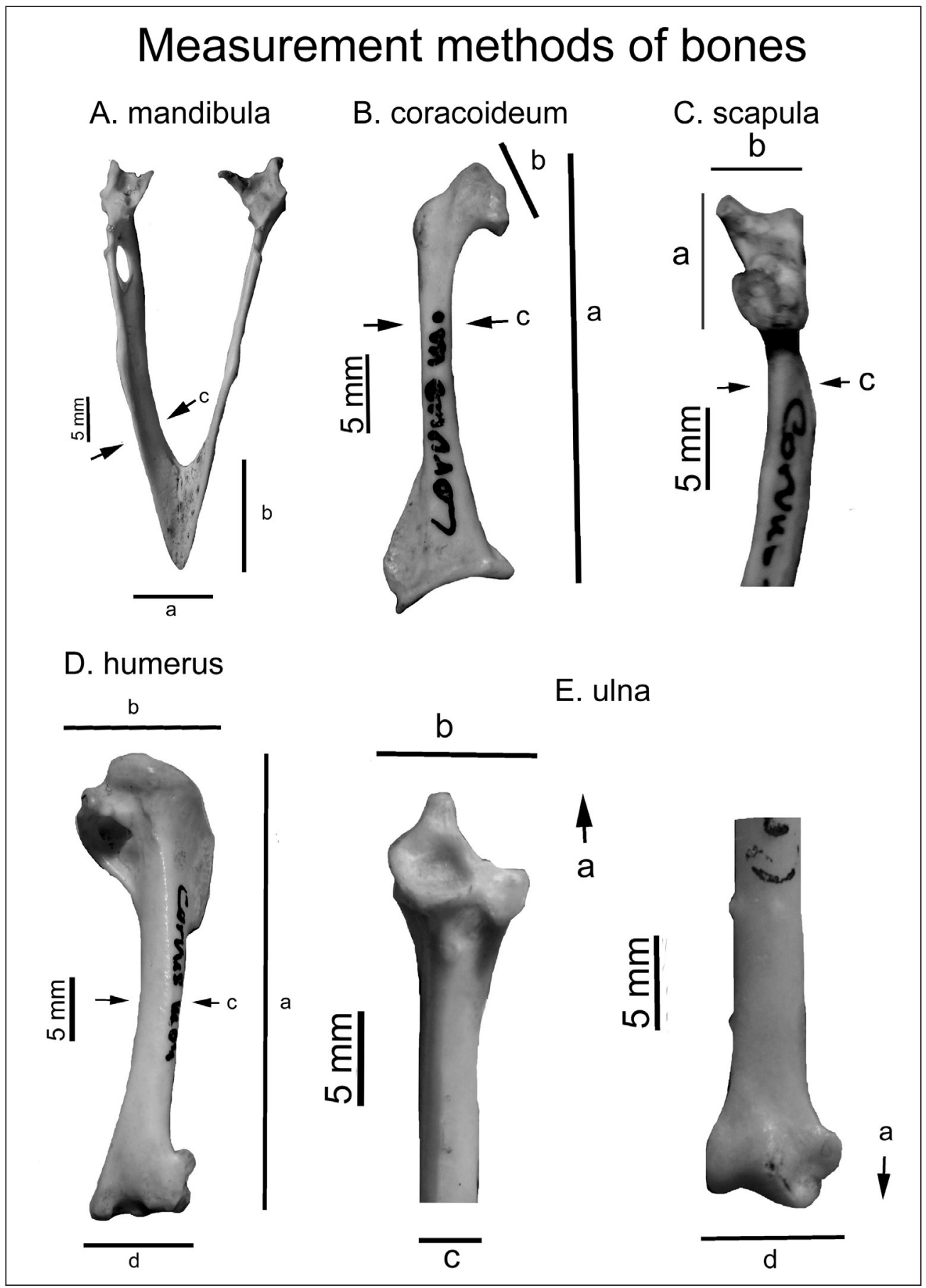

Figure 2 Measurement methods of bones I.

2. ábra A csontok mérési módja 


\section{Measurement methods of bones II.}

F. carpometacarpus G. phalanx prox.dig.maj.
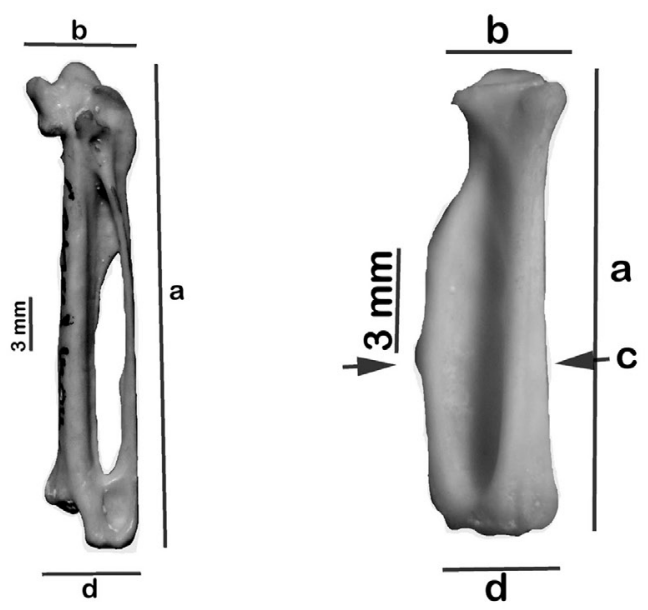

H. femur (dist)

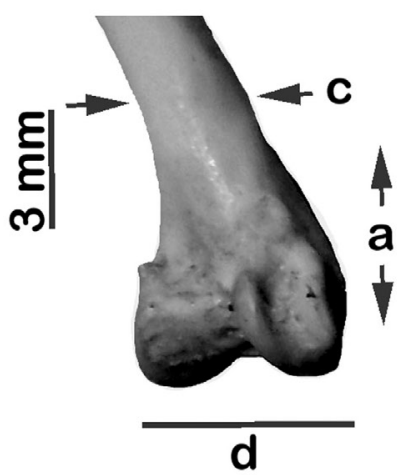

I. tibiotarsus (dist) j. tarsometatarsus (dist)

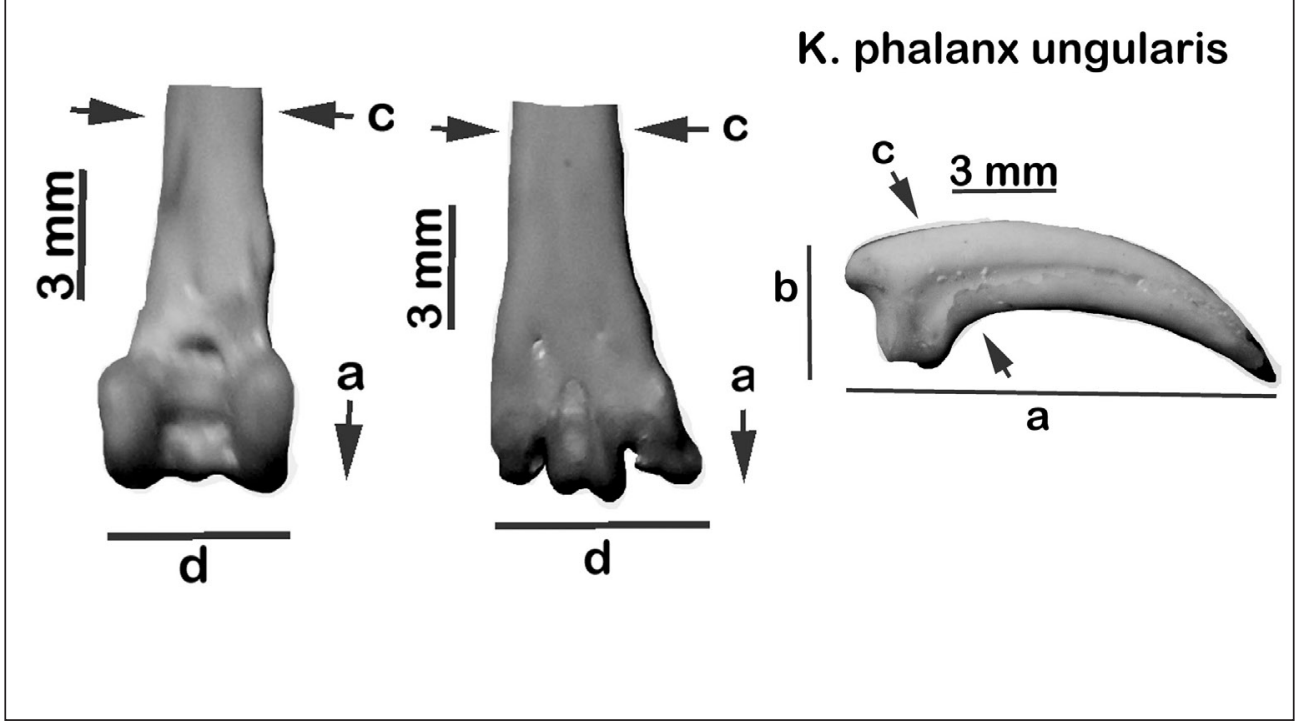

Figure 3 Measurement methods of bones II.

3. ábra A csontok mérési módja 


\section{Mandibula}

a. the end of the beak:
A.
B.
C.
D.
E.
F.
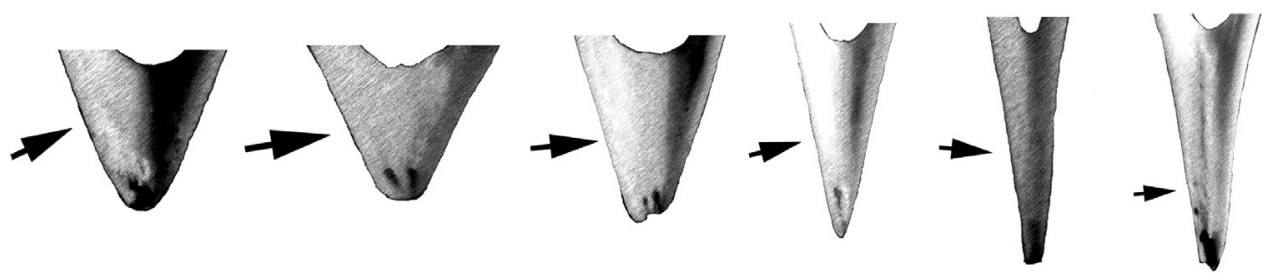

b. the slot of the beak:
A.
B.
C.
D.
E.
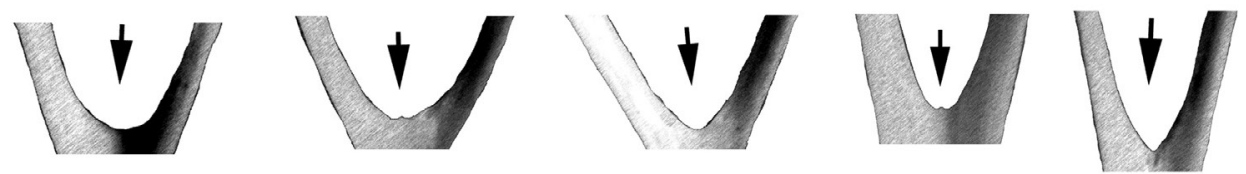

F.

G.

H.

I.
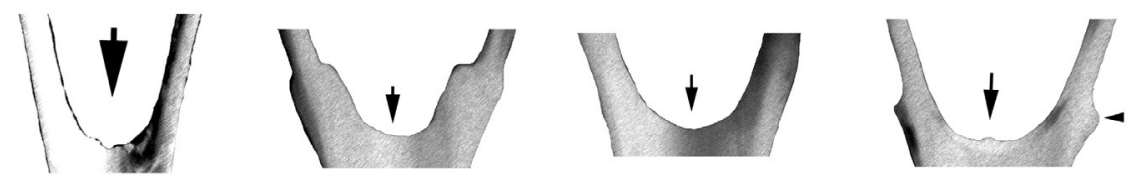

\section{c. the stem of the beak:}
A.
B.
C.
D.
E.
F.
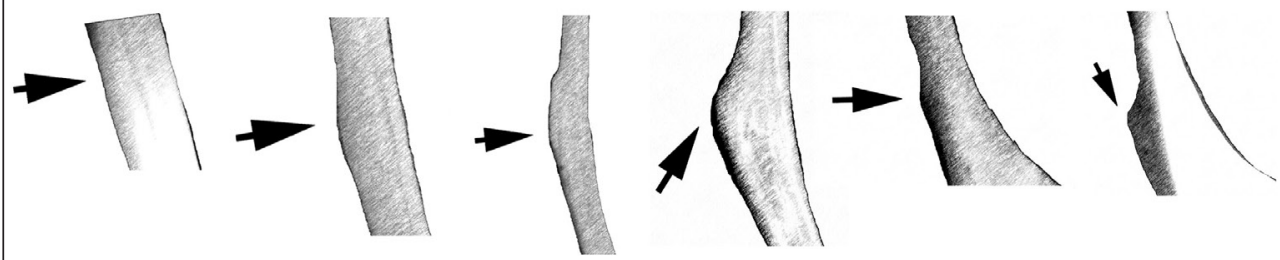

Figure 4 Mandibula coding sample ( $a$ - the end of the beak; $b$ - the slot of the beak; $c$ - the stem of the beak)

4. ábra Alsó állkapocs kódolási minta ( $\mathrm{a}$ - a csőr vége; $\mathrm{b}$ - a csőr bemélyedése; c - a csőr szára) 


\section{Coracoideum}

a. the shape of the processus acrocoracoidalis:

A

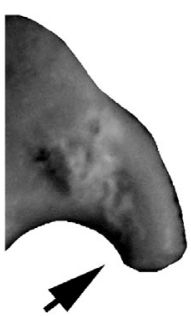

B

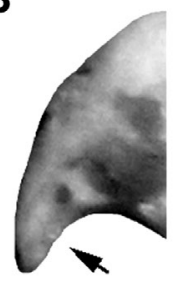

C

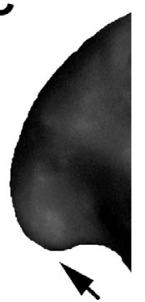

D

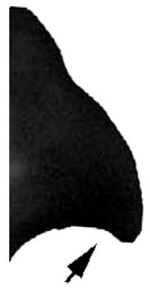

b. the shape of the acrocoracoideum:

A

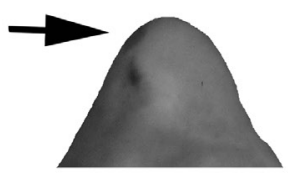

B

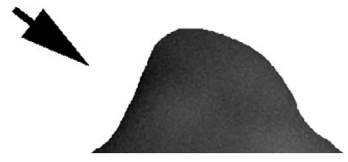

c. the form of the sulcus musculus supracoracoidalis:

A

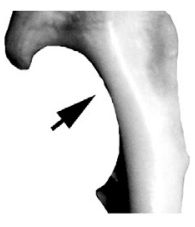

B

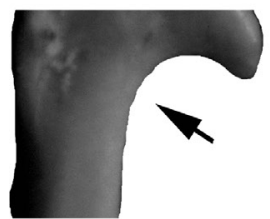

C

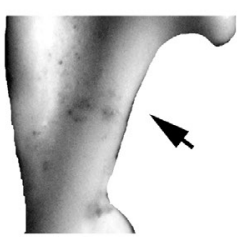

d. the existence of the processus procoracoidalis:

A

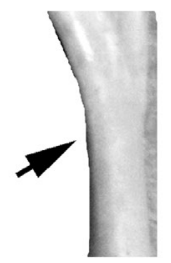

B

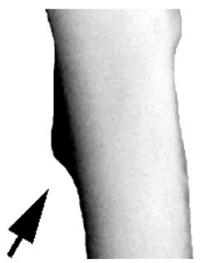

C

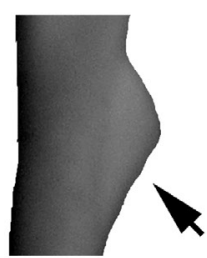

Figure 5 Coracoideum coding sample ( $\mathrm{a}$ - the shape of the processus acrocoracoidalis; $\mathrm{b}$ - the shape of the acrocoracoideum; c - the form of the sulcus musculus supracoracoidei; $\mathrm{d}$ - the existence of the processus procoracoidalis)

5. ábra Hollócsőrcsont kódolási minta (a - a processus acrocoracoidalis alakja; b - az acrocoracoideum alakja; c - a sulcus musculus supracoracoidei formája; $d$ - a processus procoracoidalis léte) 


\section{Scapula}

a. the lenght of the branches of the acromion:

A

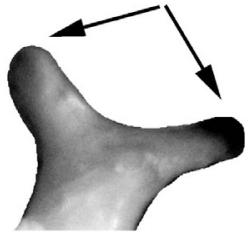

B

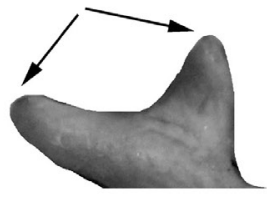

b. the similarity of the braches of the acromion:

A

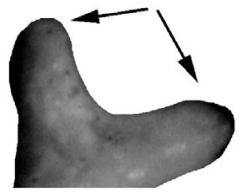

B

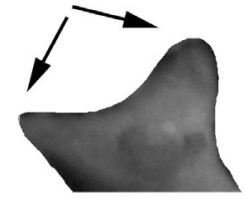

C. the shape of the apex dorsalis:

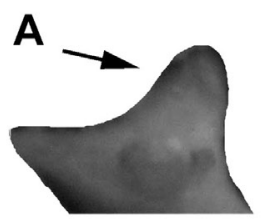

B

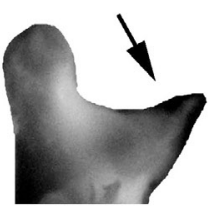

C

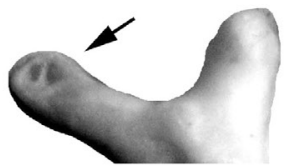

D

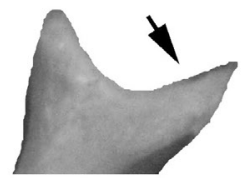

d. the shape of the apex lateralis:

A

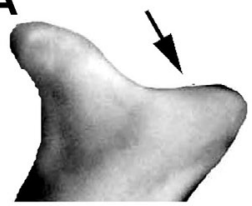

B

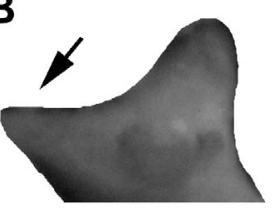

C

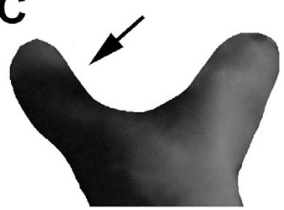

D

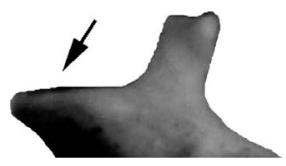

e. the character of the pit between the branches:

A

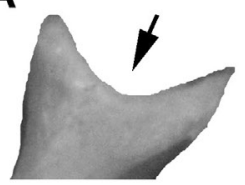

B

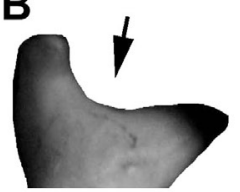

C

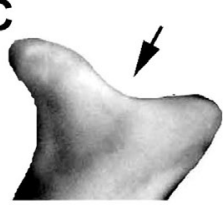

D

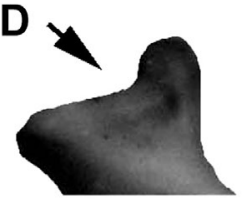

Figure 6 Scapula coding sample ( $\mathrm{a}$ - the lenght of the branches of the acromion; $\mathrm{b}$ - the similarity of the branches of the acromion; $\mathrm{c}$ - the shape of the apex dorsalis; $\mathrm{d}$ - the shape of the apex lateralis; $\mathrm{e}$ - the character of the pit between the branches)

6. ábra Lapocka kódolási minta (a - az acromion szárainak a hossza; b - az acromion szárainak a hasonlósága; c - a hátoldali nyúlvány alakja; d - az oldalsó nyúlvány alakja; e - a szárak közti mélyedés formája) 


\section{Humerus (epiph. prox.)}

a. the shape of the tuberculum ventrale:

A

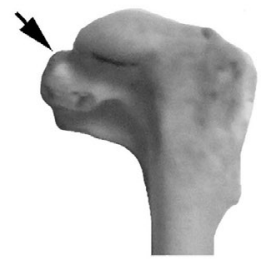

B

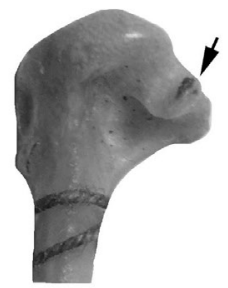

b. the form of the end of the crista bicipitalis

A

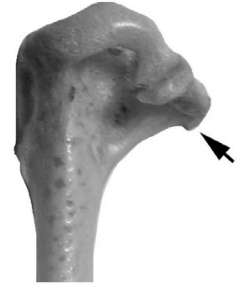

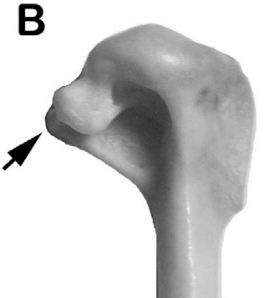

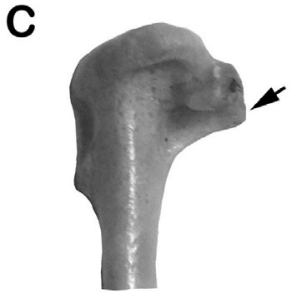

c. the form of the distal edge of the crista bicipitalis

A

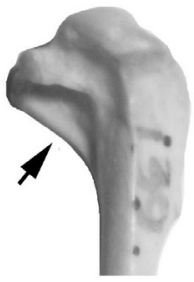

B

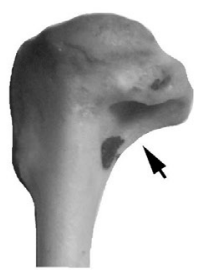

d. the character of the fossa pneumotricipitalis:

A

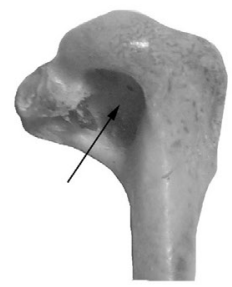

B

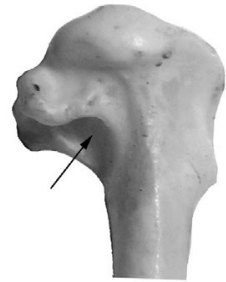

Figure 7 Humerus (epiph. prox.) coding sample ( $a$ - the shape of the tuberculum ventrale; $b$ - the form of the crista bicipitalis; $\mathrm{c}$ - the form of the distal edge of the crista bicipitalis; $\mathrm{d}$ - the character of the fossa pneumotricipitalis)

7. ábra Felkarcsont (prox. epifízis) kódolási minta ( $\mathrm{a}$ - a tuberculum ventrale alakja; b - a crista bicipitalis formája; c - a crista bicipitalis disztális élének a formája; $\mathrm{d}$ - a fossa pneumotricipitalis jellege) 


\section{Humerus (epiph.dist.)}

a. the form of the tuberculum supracondylare ventrale:
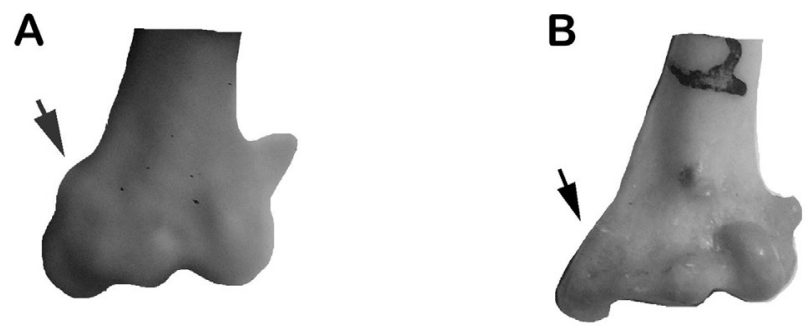

b. the shape of the processus flexorius:
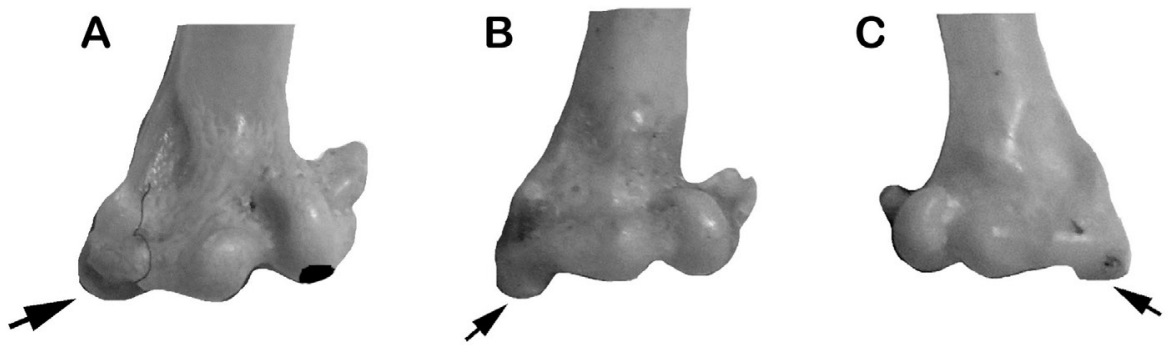

c. the form of the processus supracondylaris dorsalis:
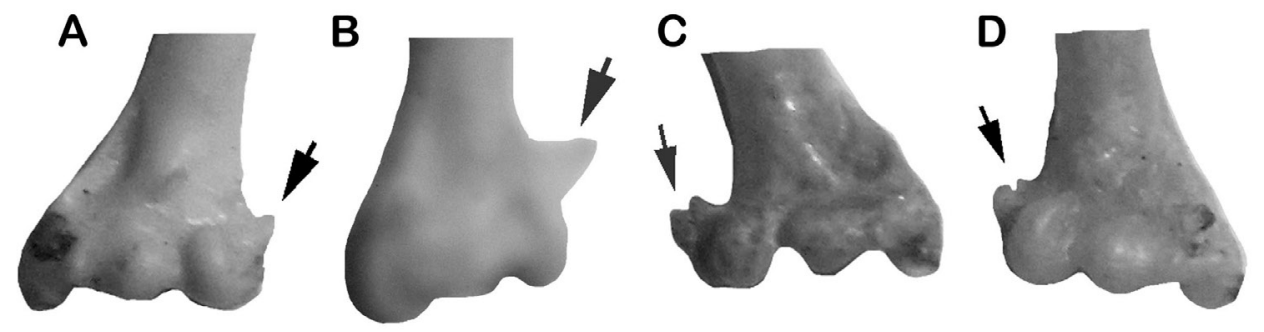

Figure 8 Humerus (epiph. dist.) coding sample (a - the form of the tuberculum supracondylare ventrale; $\mathrm{b}$ - the shape of the processus flexorius; $\mathrm{c}$ - the form of the processus supracondylaris dorsalis)

8. ábra Felkarcsont (diszt. epifízis) kódolási minta (a - a tuberculum supracondylare ventrale formája; b - a processus flexorius alakja; c - a processus supracondylaris dorsalis formája) 


\section{Ulna (epiph. prox.)}

a. the shape of the oleocranon:
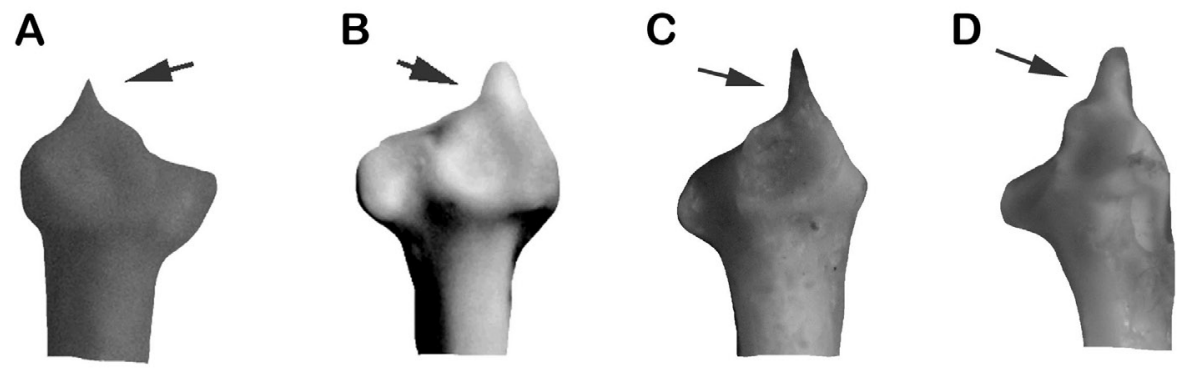

b. the form of the cotyla dorsalis:

A

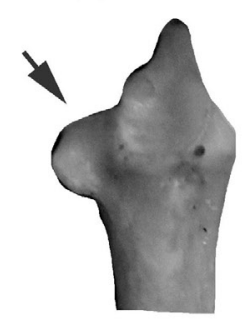

B

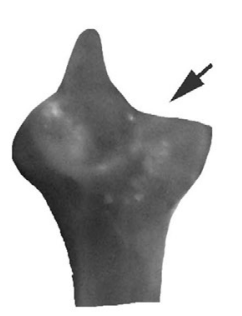

C

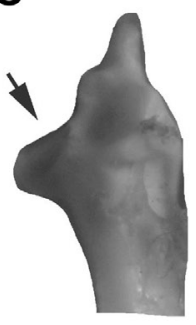

D

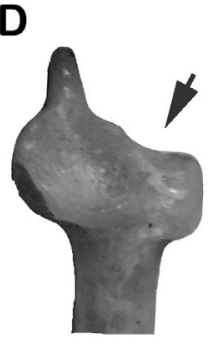

E

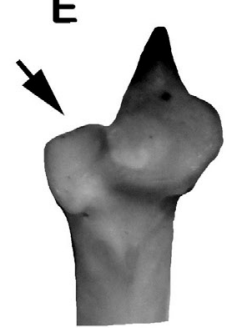

c. the existence of the tuberculum ligamentum collateralis ventralis:

A

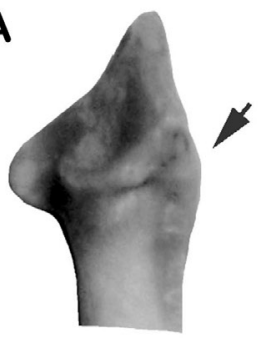

B

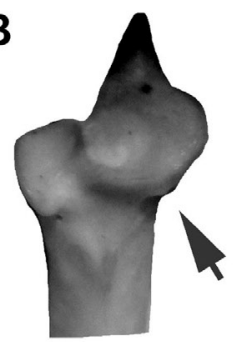

Figure 9 Ulna (epiph. prox.) coding sample ( $\mathrm{a}$ - the shape of the olecranon; $\mathrm{b}$ - the form of the cotyla dorsalis; $\mathrm{c}$ - the existence of the tuberculum ligamentum collateralis ventralis)

9. ábra Singcsont (prox. epifízis) kódolási minta (a - az olecranon alakja; b - a cotyla dorsalis formája; c - a tuberculum ligamentum collateralis ventralis léte) 


\section{Ulna (epiph. dist.)}

a. the shape of the condylus dorsalis:

A

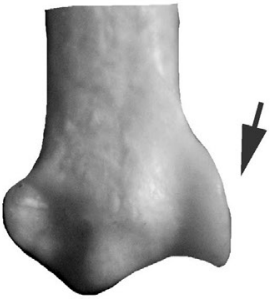

B

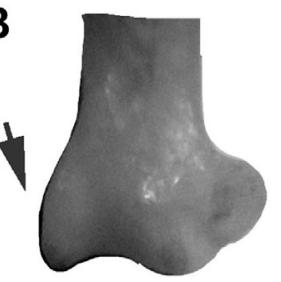

C

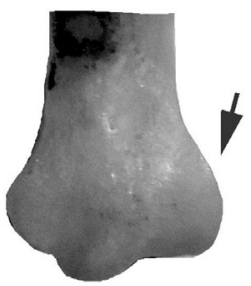

b. the form of the sulcus intercondylaris:

A

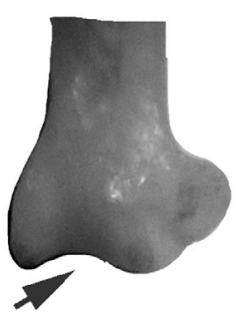

B

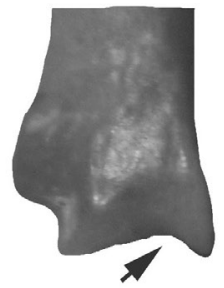

C

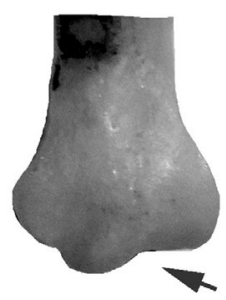

c. the shape of the condylus ventralis:

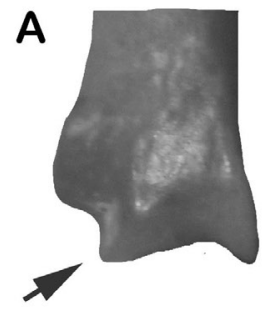

B

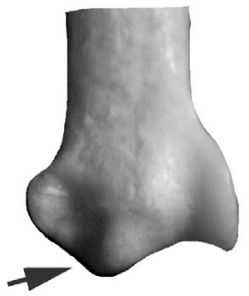

C

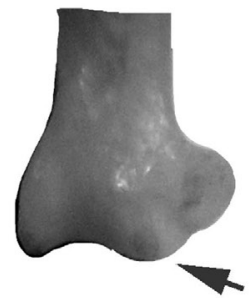

d. the form of the tuberculum carpale:

A

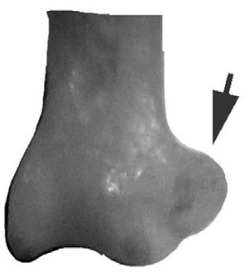

B

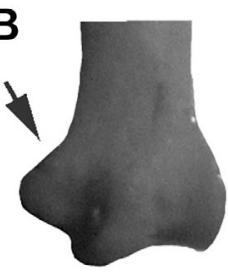

C

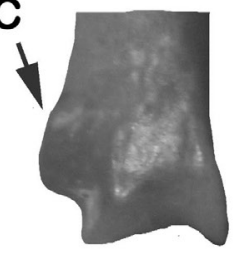

D

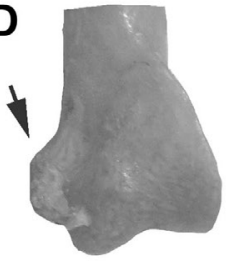

Figure 10 Ulna (epiph. dist.) coding sample ( $\mathrm{a}$ - the shape of the condylus dorsalis; $\mathrm{b}$ - the form of the sulcus intercondylaris; $\mathrm{c}$ - the shape of the condylus ventralis; $\mathrm{d}$ - the form of the tuberculum carpale)

10. ábra Singcsont (diszt. epifízis) kódolási minta (a - a condylus dorsalis alakja; b - a sulcus intercondylaris formája; $\mathrm{c}$ - a condylus ventralis alakja; $\mathrm{d}$ - a tuberculum carpale formája) 


\section{Carpometacarpus I.}

a. the form of the trochlea carpalis:

A

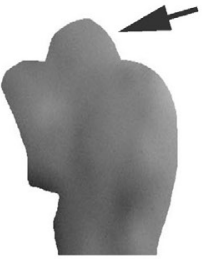

B

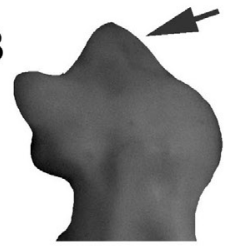

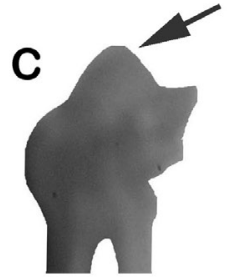

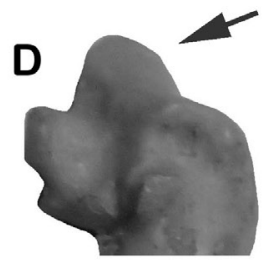

b. the shape of the processus extensorius:
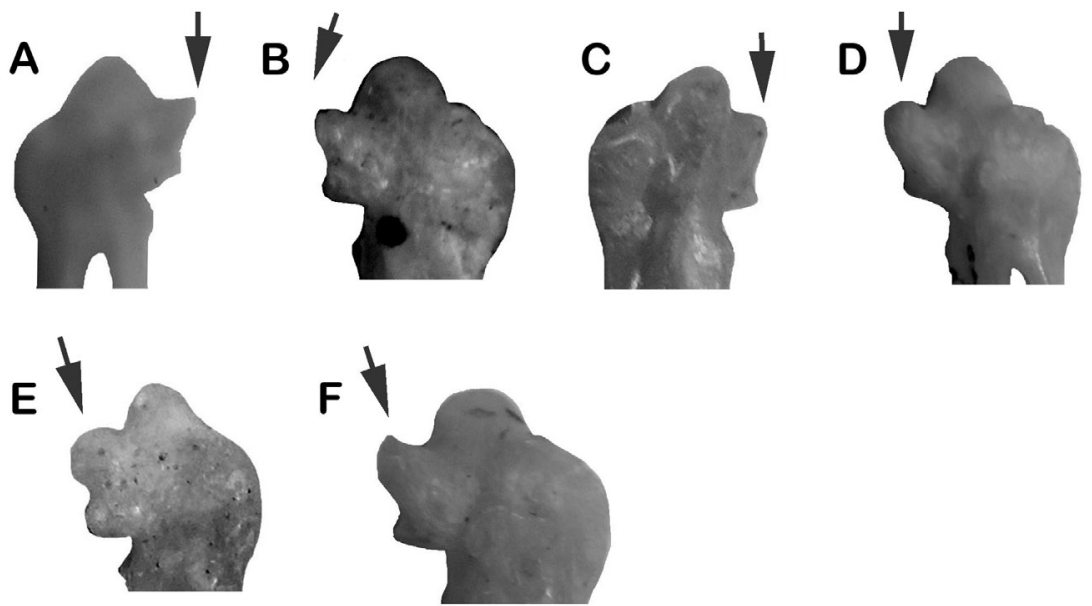

c. the form of the processus alularis:
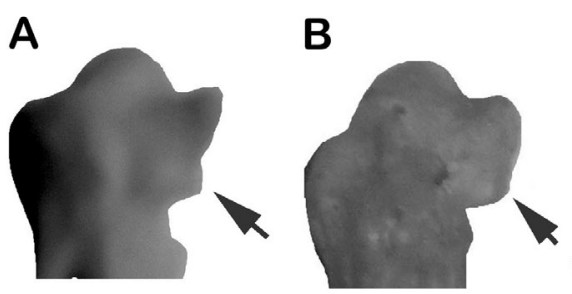

C

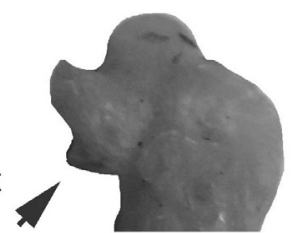

D

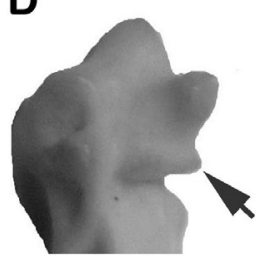

Figure 11 Carpometacarpus I. coding sample ( $\mathrm{a}$ - the shape of the a trochlea carpalis; $\mathrm{b}$ - the form of the processus extensorius; $\mathrm{c}$ - the shape of the processus alularis)

11. ábra Kézközépcsont kódolási minta I. ( $\mathrm{a}$ - a trochlea carpalis alakja; $\mathrm{b}$ - a processus extensorius formája; c - a processus alularis alakja) 


\section{Carpometacarpus II.}

d. the form of the fovea subalularis:
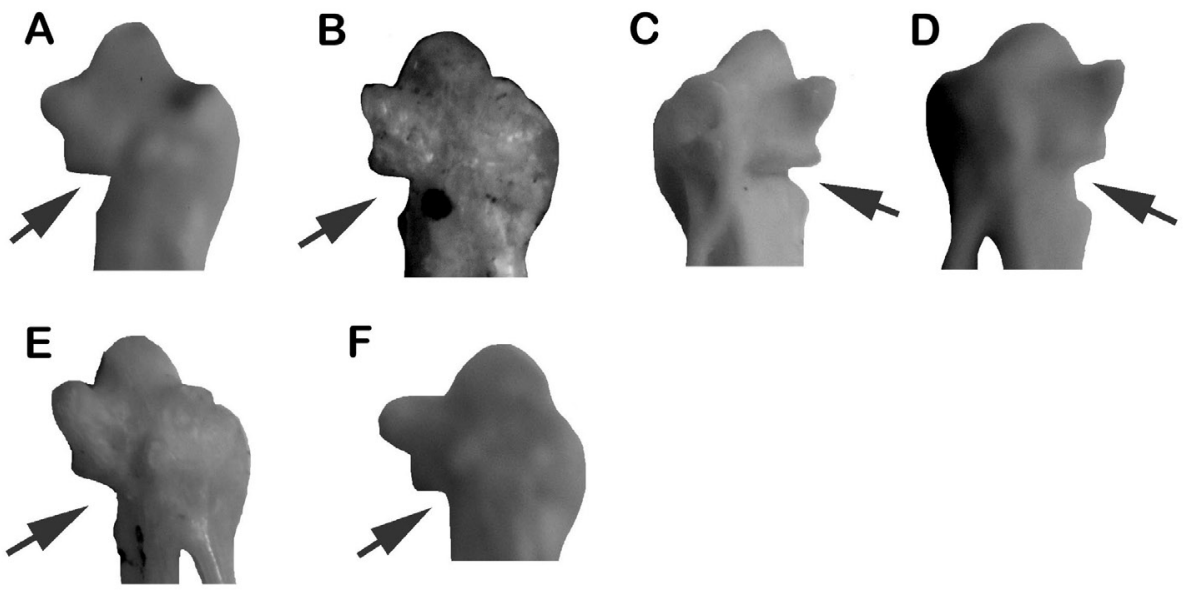

e. the shape of the protuberantia metacarpalis:
A
B
C
D
$\mathbf{E}$
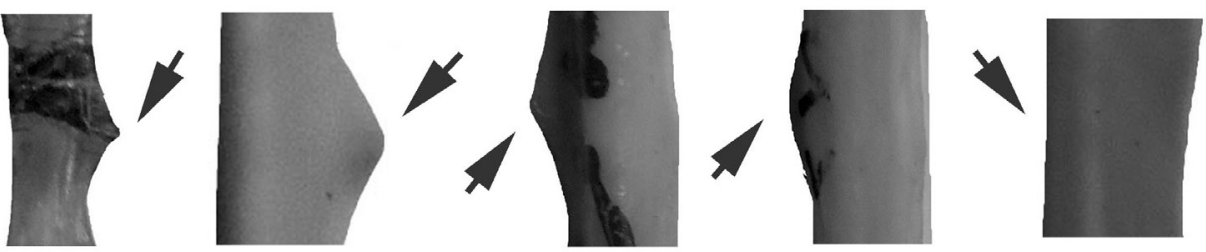

f. the shape of the facies articularis digitalis minor:

A

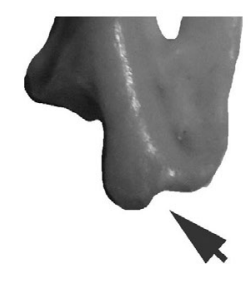

B

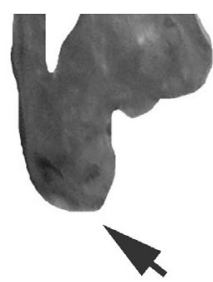

C

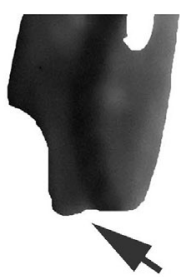

D

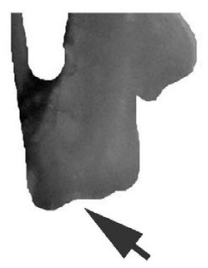

E

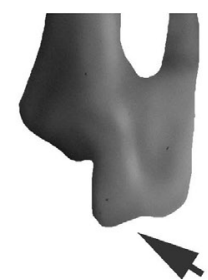

Figure 12 Carpometacarpus II. coding sample ( $\mathrm{d}$ - the form of the fovea subalularis; $\mathrm{e}$ - the character of the protuberantia metacarpale majus; $f$ - the shape of the facies articularis digitale minor);

12. ábra Kézközépcsont kódolási minta ( $\mathrm{d}$ - a fovea subalularis formája; e - a protuberantia metacarpale majus jellege; $\mathrm{f}$ - a facies articularis digitale minor alakja) 


\section{Phalanx proximalis digiti majoris}

a. the form of the facies articularis metacarpalis:
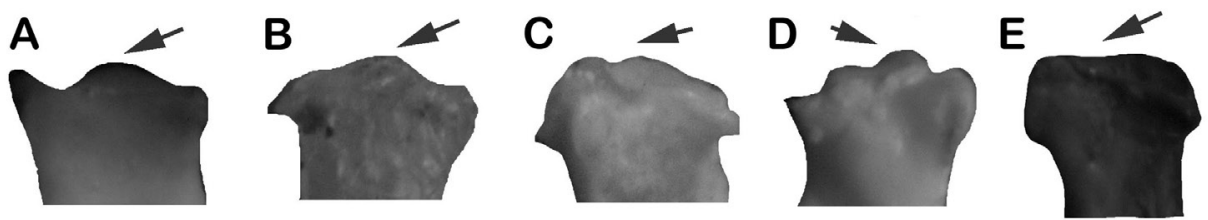

b. the shape of the tuberculum ventralis:
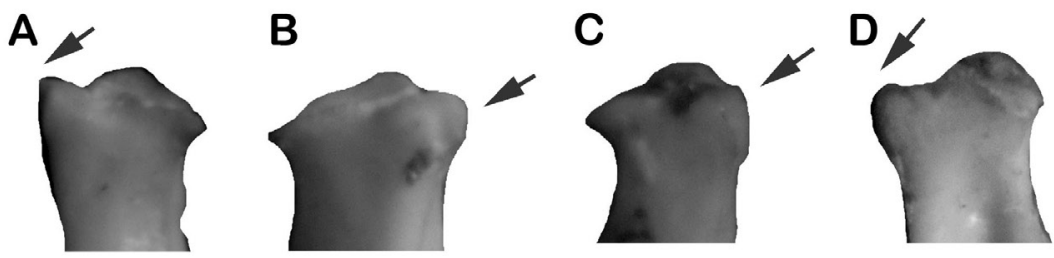

c. the shape of the tuberculum dorsalis:
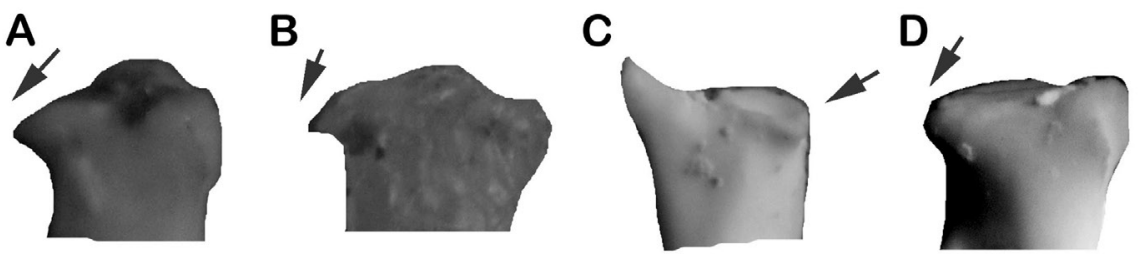

d. the character of the margo dorsalis:

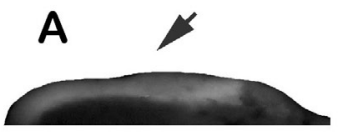

B

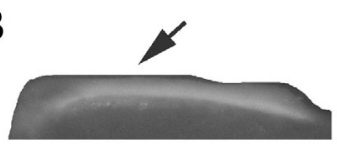

C

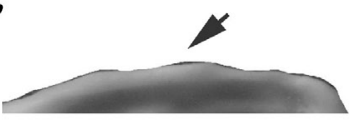

e. the form of the facies articularis phalangealis:

A

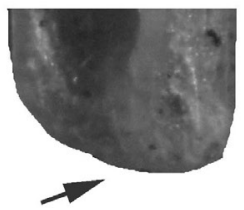

B

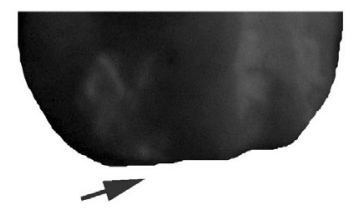

C

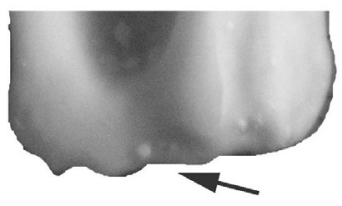

Figure 13 Phalanx proximalis digiti majoris coding sample (a - the form of the margo proximalis; $\mathrm{b}$ - the form of the tuberculum ventralis; $\mathrm{c}$ - the form of the tuberculum dorsalis; $\mathrm{d}$ - the character of the margo dorsalis; e - the character of the margo distalis)

13. ábra Kézujjperc kódolási minta ( $\mathrm{a}$ - a margo proximalis formája; $\mathrm{b}$ - a tuberculum ventralis formája; c -a tuberculum dorsalis formája; $\mathrm{d}$ - a margo dorsalis jellege; e - a margo distalis jellege) 


\section{Femur (epiphysis distalis)}

a. the shape of the condylus medialis:
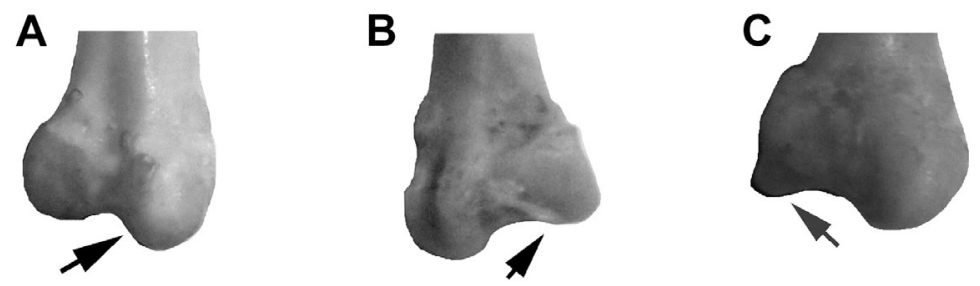

b. the character of the sulcus intercondylaris:
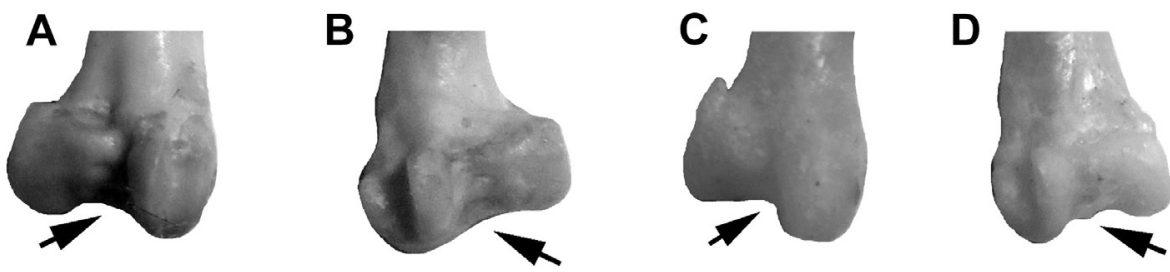

C. the shape of the condylus lateralis:

A

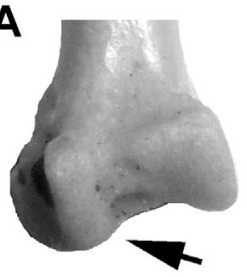

B

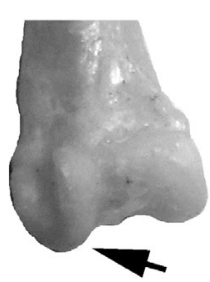

C

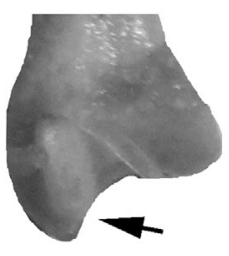

d. the form of the epicondylus medialis:

A

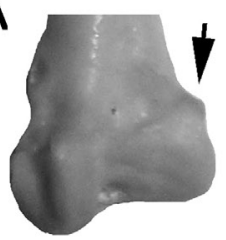

B

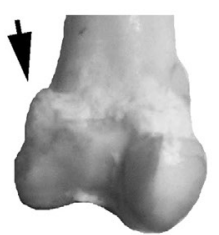

C

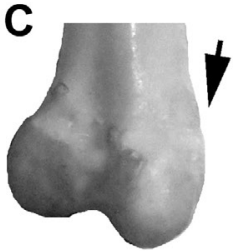

e. the form of the epicondylus lateralis:

A

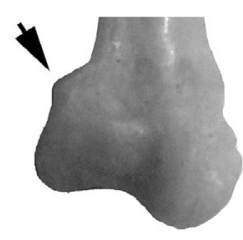

B

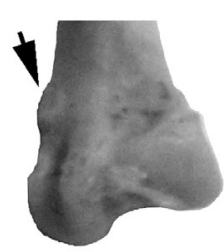

C

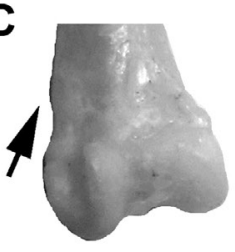

D

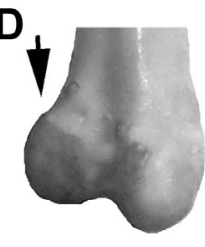

Figure 14 Femur (epiph.dist.) coding sample ( $\mathrm{a}$ - the shape of the condylus medialis; $\mathrm{b}$ - the character of the sulcus intercondylaris; $c$ - the shape of the condylus lateralis; $d$ - the form of the epicondylus medialis; e - the form of the epicondylus lateralis)

14. ábra Combcsont (diszt. epifízis) kódolási minta ( $\mathrm{a}$ - a condylus medialis alakja; b - a sulcus intercondylaris jellege; c - a condylus lateralis alakja; d - az epicondylus medialis formája; e - az epicondylus lateralis formája) 


\section{Tibiotarsus (epiphysis distalis)}

a. the shape of the epicondylus medialis

A

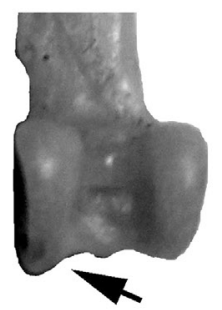

B

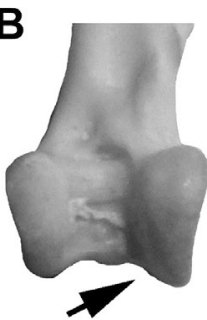

C

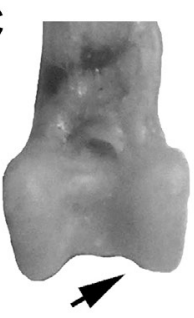

b. the shape of the epicondylus lateralis

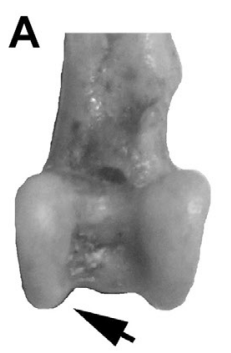

B

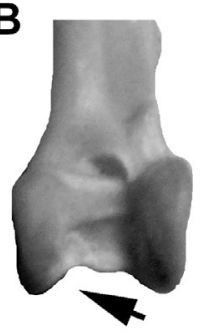

C

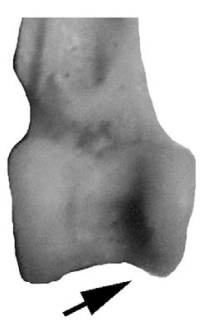

D

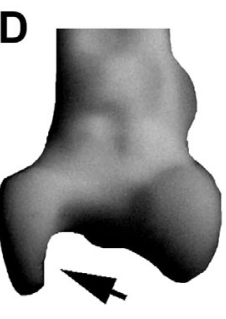

c. the character of the incisura intercondylaris:

A

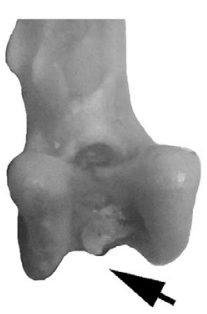

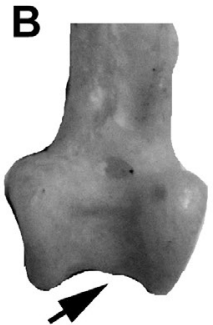

C

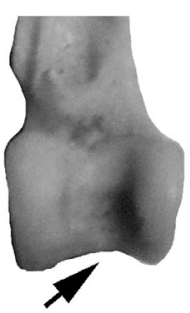

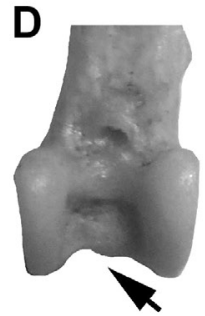

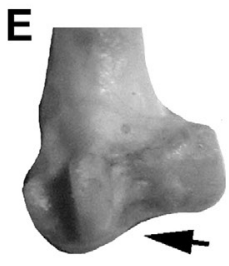

d. the form of the tuberculum retinaculi musc. fibularis:

A

4

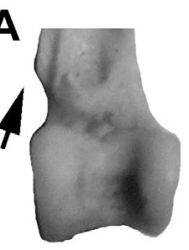

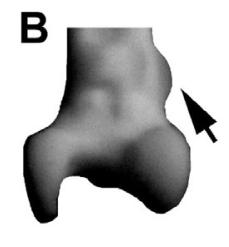
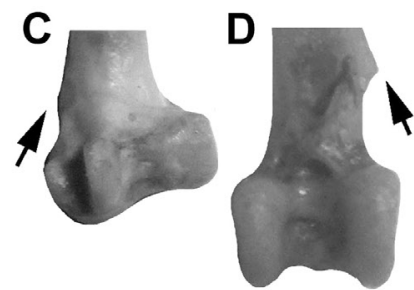

E
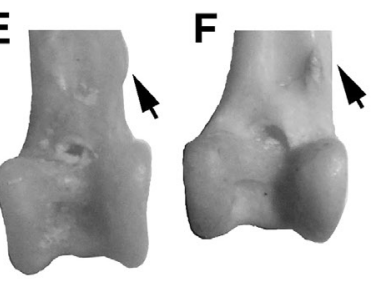

Figure 15 Tibiotarsus (epiph. dist.) coding sample ( $\mathrm{a}$ - the shape of the epicondylus lateralis; $\mathrm{b}$ - the shape of the epicondylus medialis; $\mathrm{c}$ - the form of the incisura intercondylaris; $\mathrm{d}$ - the character of the tuberositas retinaculi)

15. ábra Lábszárcsont (diszt. epifízis) kódolási minta (a - az epicondylus lateralis alakja; b - az epicondylus medialis alakja; c - az incisura intercondylaris formája; $\mathrm{d}$ - a tuberositas retinaculi jellege) 


\section{Tarsometatarsus (epiphysis distalis)}

a. the character of the margo medialis:

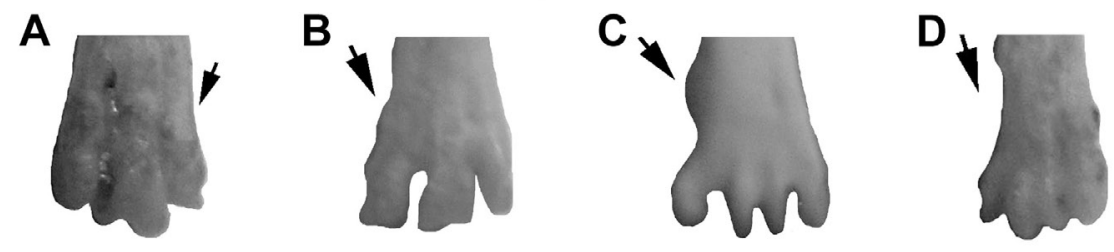

b. the shape of the trochlea metatarsi II.:
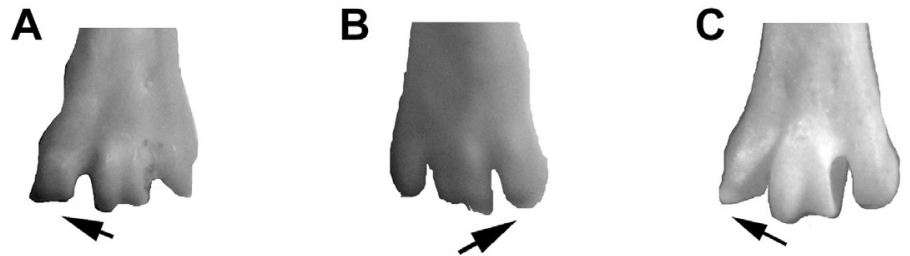

C. the shape of the trochlea metatarsi III.:

A

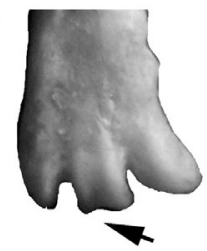

B

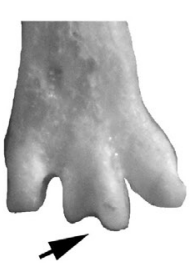

C

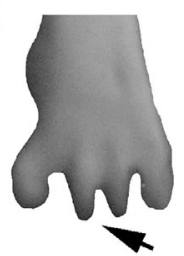

d. the shape of the trochlea metatarsi IV.:
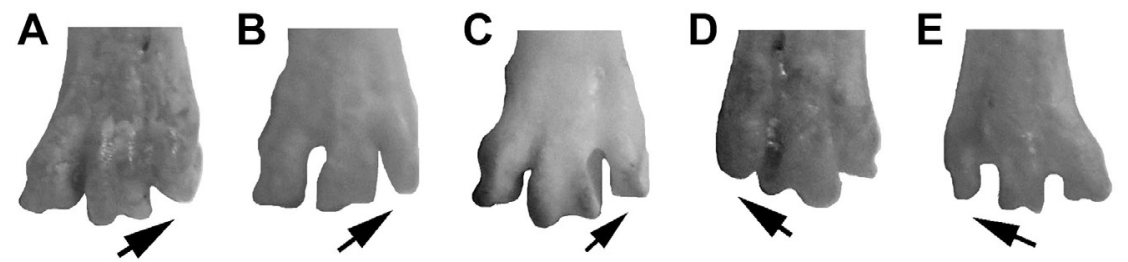

e. the form of the incisura intertrochlearis medialis and lateralis:

A

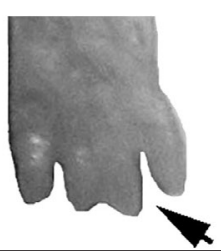

B

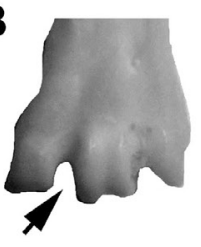

A

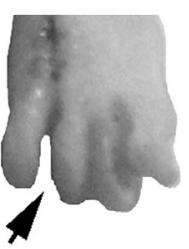

B

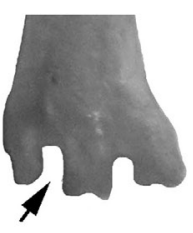

Figure 16 Tarsometatarsus (epiph. dist.) coding sample (a - the character of the margo medialis; $\mathrm{b}$ - the shape of the troclea metatarsi II.; c - the shape of the trochlea metatarsi III.; $\mathrm{d}$ - the shape of the trochlea metatarsi IV.; e - the form of the incisura intertrochlearis medialis; $\mathrm{f}$ - the form of the incisura intertrochlearis lateralis)

16. ábra Csüd (diszt. epifízis) kódolási minta ( $\mathrm{a}$ - a margo medialis jellege; b - a troclea metatarsi Il. alakja; c - a troclea metatarsi III. alakja; d - a troclea metatarsi IV. alakja; e - az incisura intertrochlearis medialis formája; $\mathrm{f}$ - az incisura intertrochlearis lateralis formája) 


\section{Phalanx ungularis}

a. the character of the tuberculum extensorium:

A

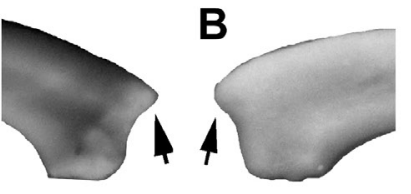

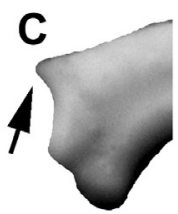
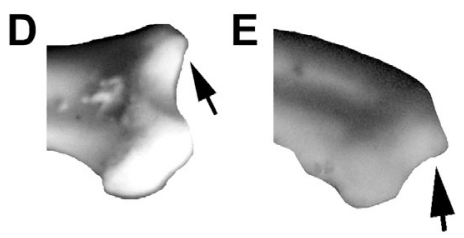

b. the form of the cotyla articularis:
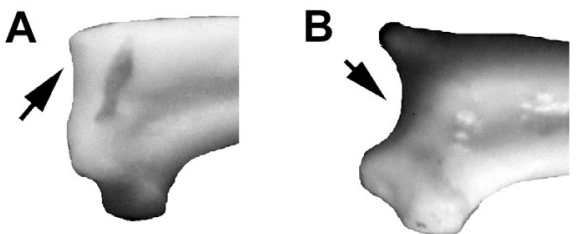

c. the character of the tuberculum flexorium:
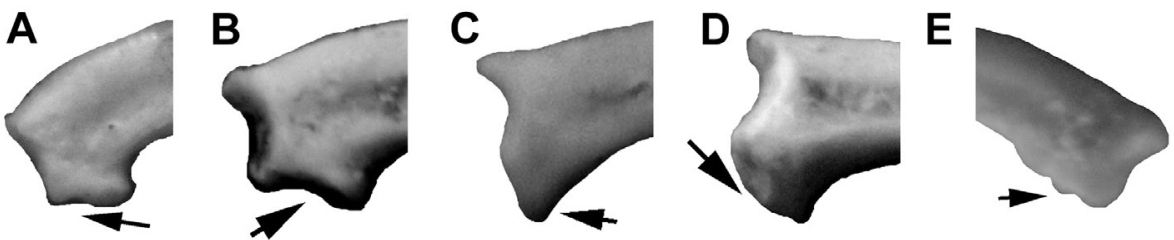

d. the curvature of the corpus:

A

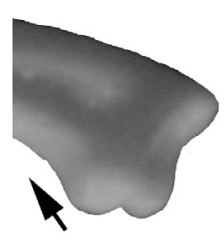

B

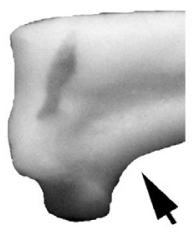

C

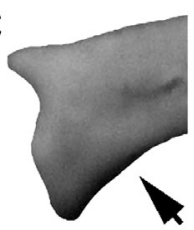

e. the shape of the apex:

A

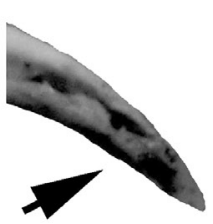

B

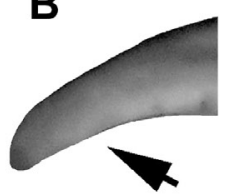

C

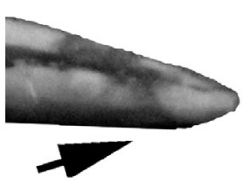

Figure 17 Phalanx ungularis coding sample (a - the direction and the form of the tuberculum extensorium; $\mathrm{b}$ - the character of the cotyla articularis; $\mathrm{c}$ - the shape of the tuberculum flexorium; $\mathrm{d}$ - the elbow of the margo plantaris; e -the form of the apex)

17. ábra Karomcsont kódolási minta ( $\mathrm{a}$ - a tuberculum extensorium iránya és formája; $\mathrm{b}$ - a cotyla articularis jellege; c - a tuberculum flexorium alakja; $\mathrm{d}$ - a margo plantaris görbülete; e - az apex alakja) 


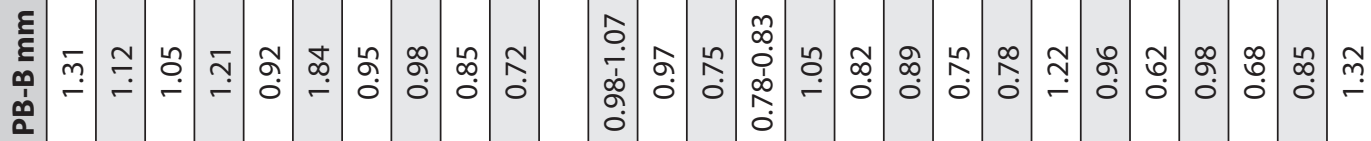

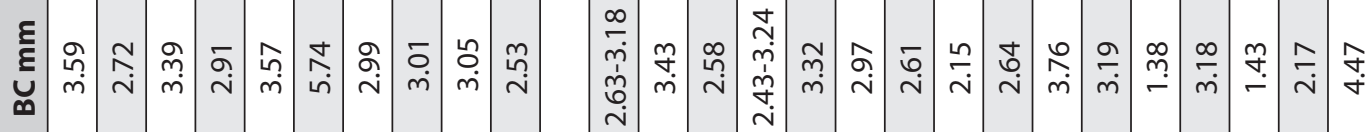

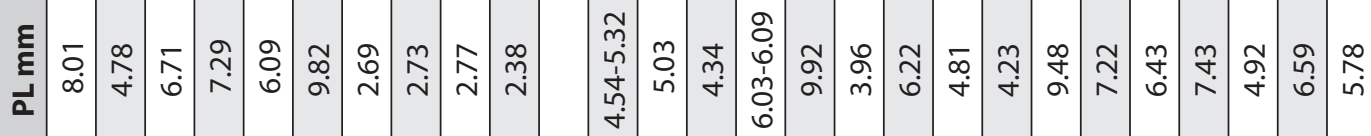

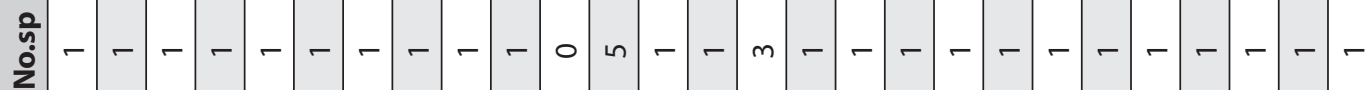

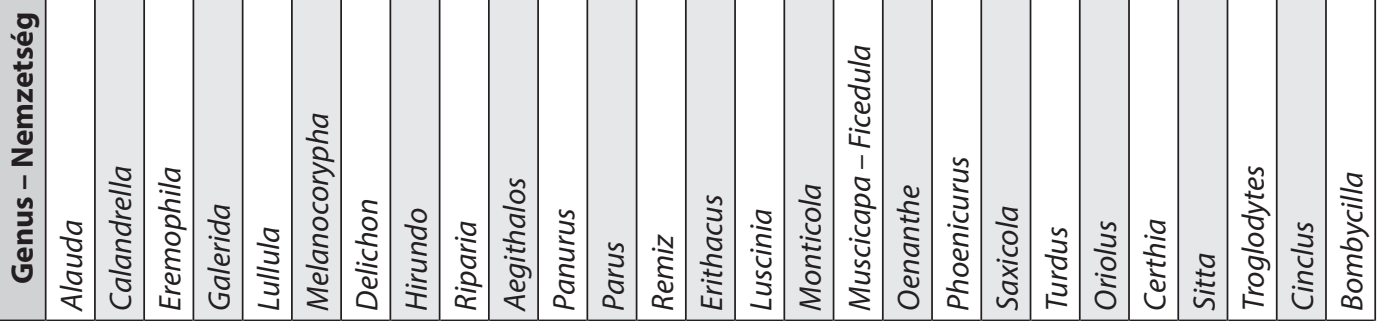

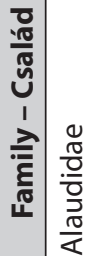

\begin{tabular}{|c|}
\hline $\begin{array}{l}\frac{\pi}{\pi} \\
\frac{\pi}{\pi} \\
\frac{0}{2}\end{array}$ \\
\hline
\end{tabular}

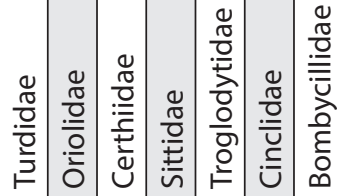




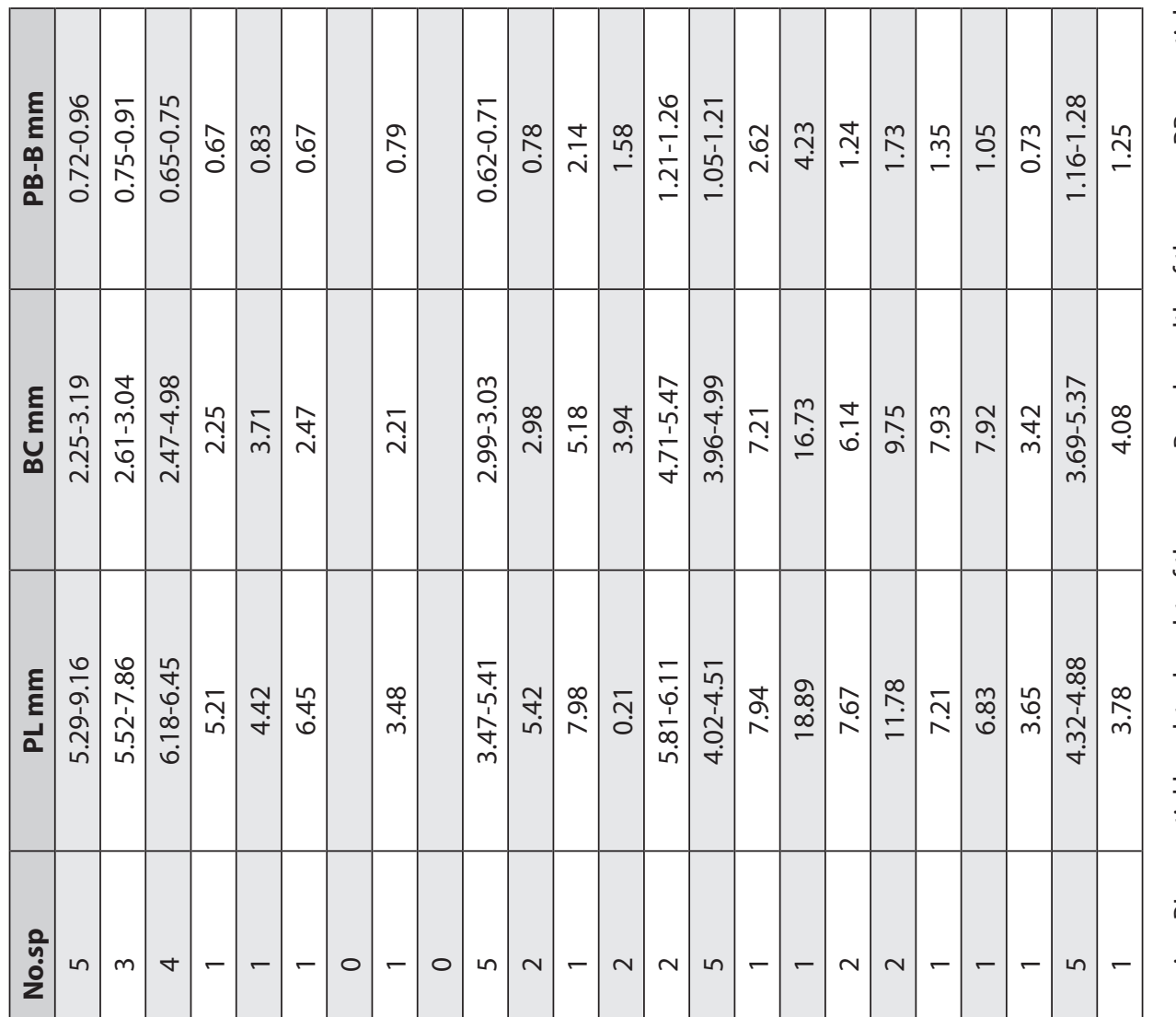

ত

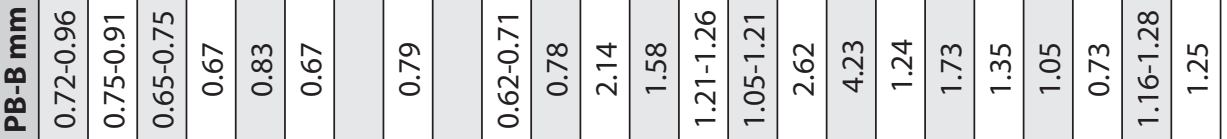

II

$\cong$ :

ฮ

䨗

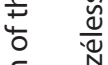

吾

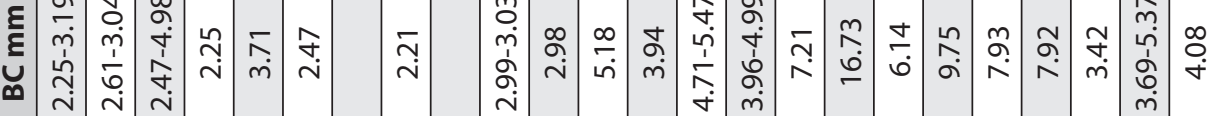

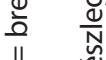

$\breve{\square}$

艾

\%

饮

.

总 :

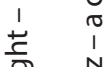

등

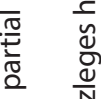

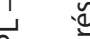

a

包

घे

ㅎำ

ब

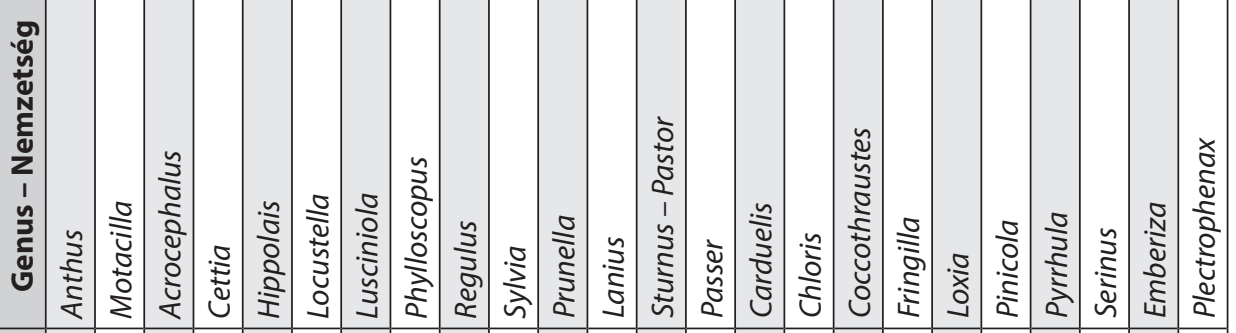

हो 흥

ह 엉

은

iे

o

원

등 홍

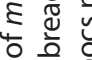

㶾

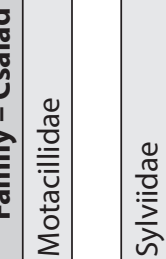

更 흥 홍

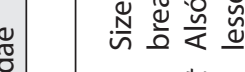




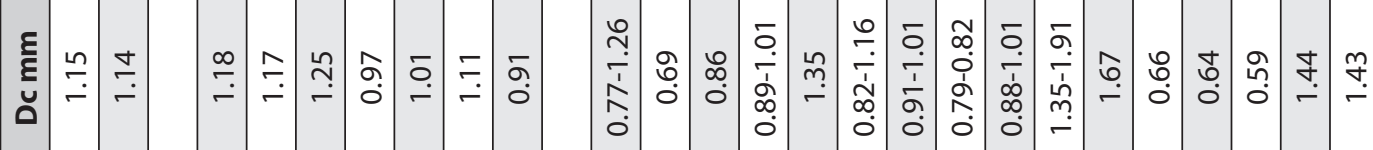

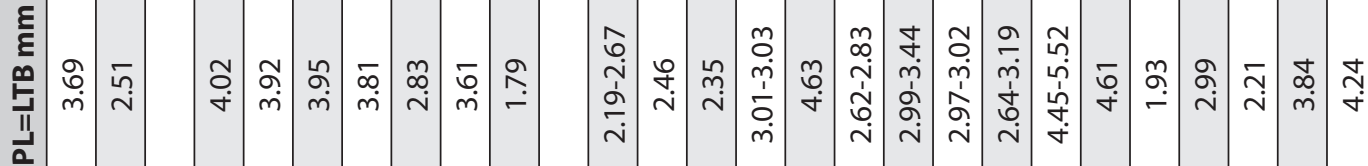

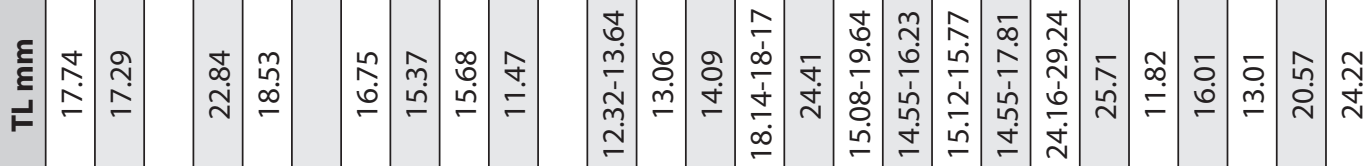

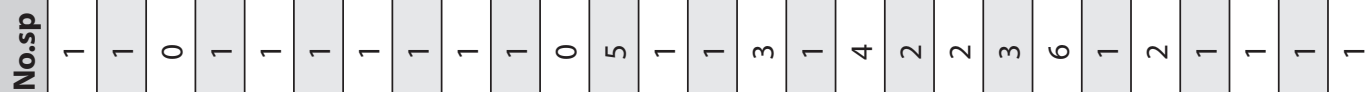

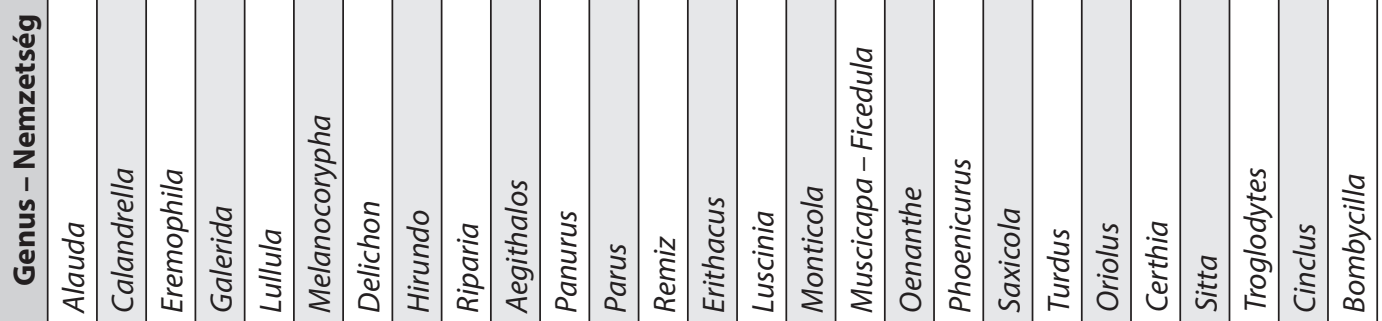

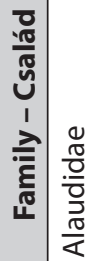

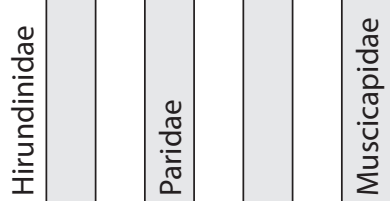




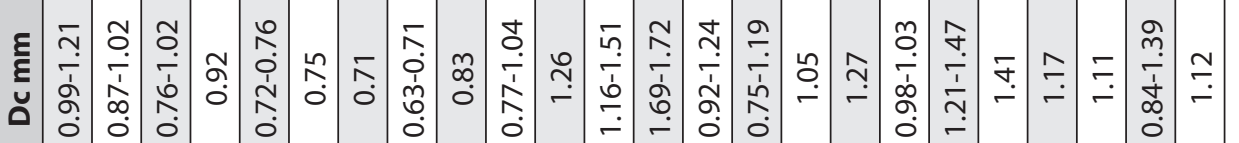

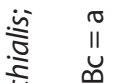

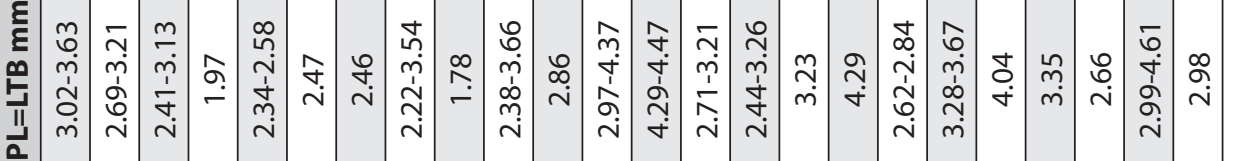
ఫิ

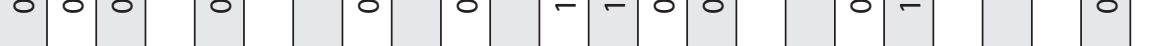

$$
\begin{array}{l|l|l|l|l} 
& & & \\
\hline
\end{array}
$$

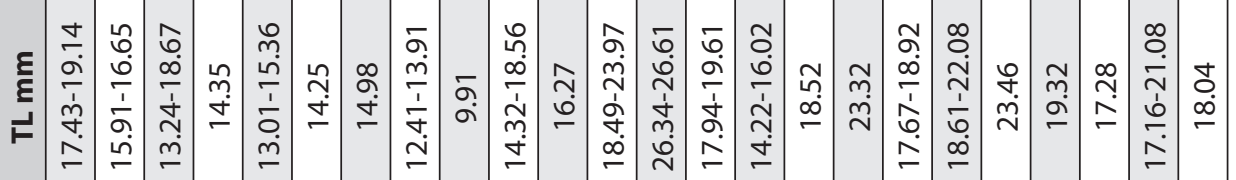

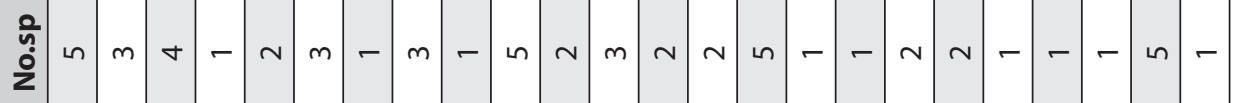
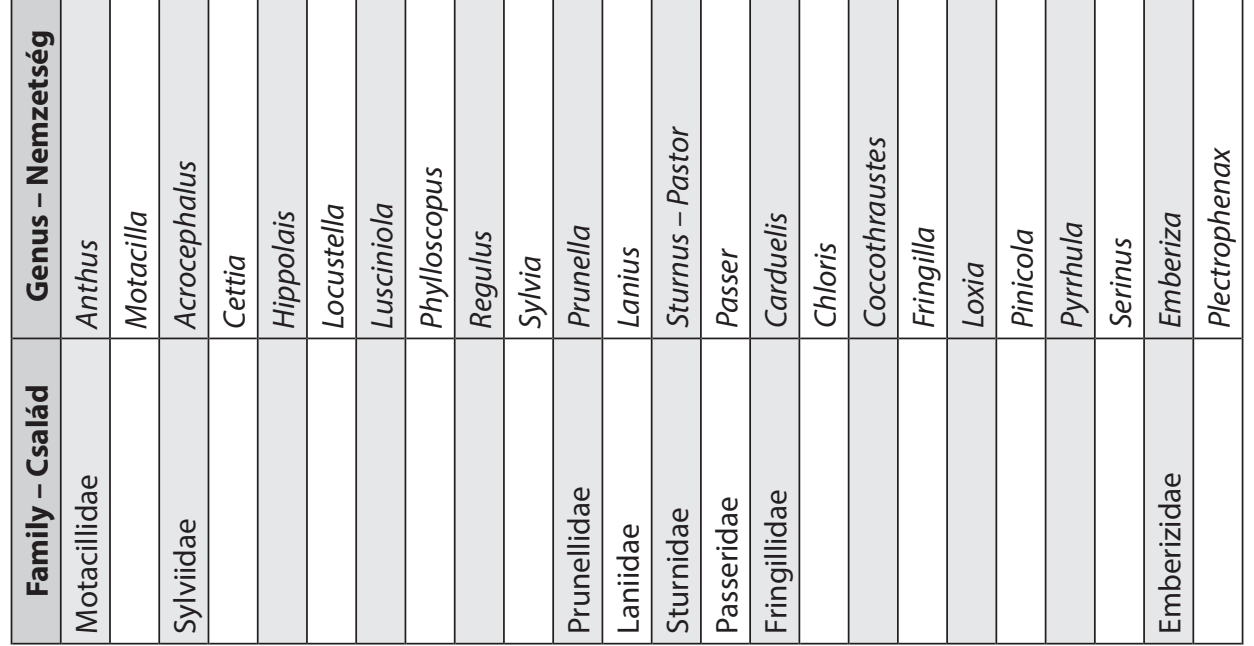


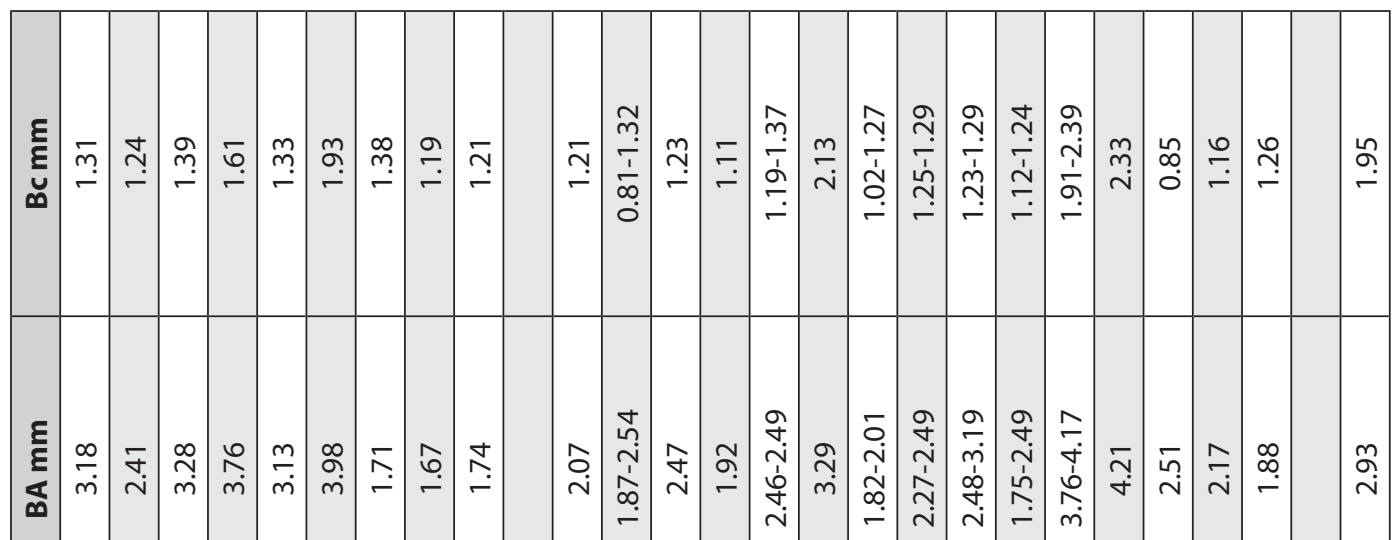

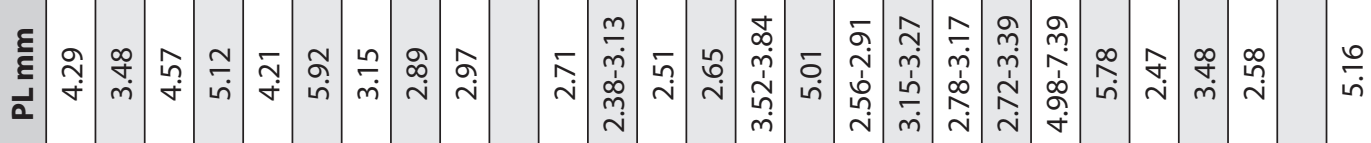
$\frac{0}{\grave{h}}$

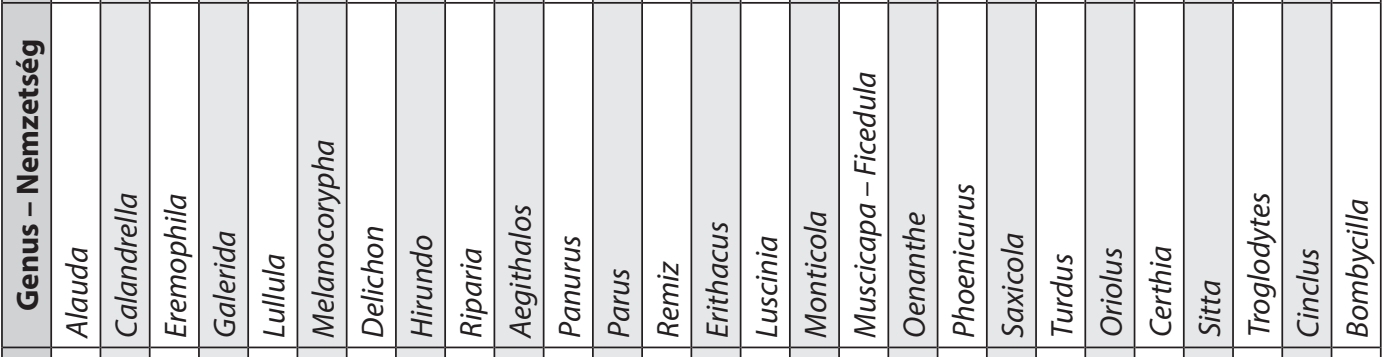

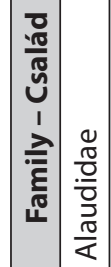

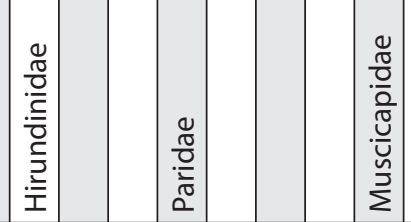

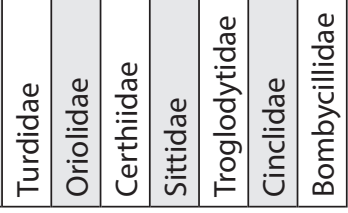




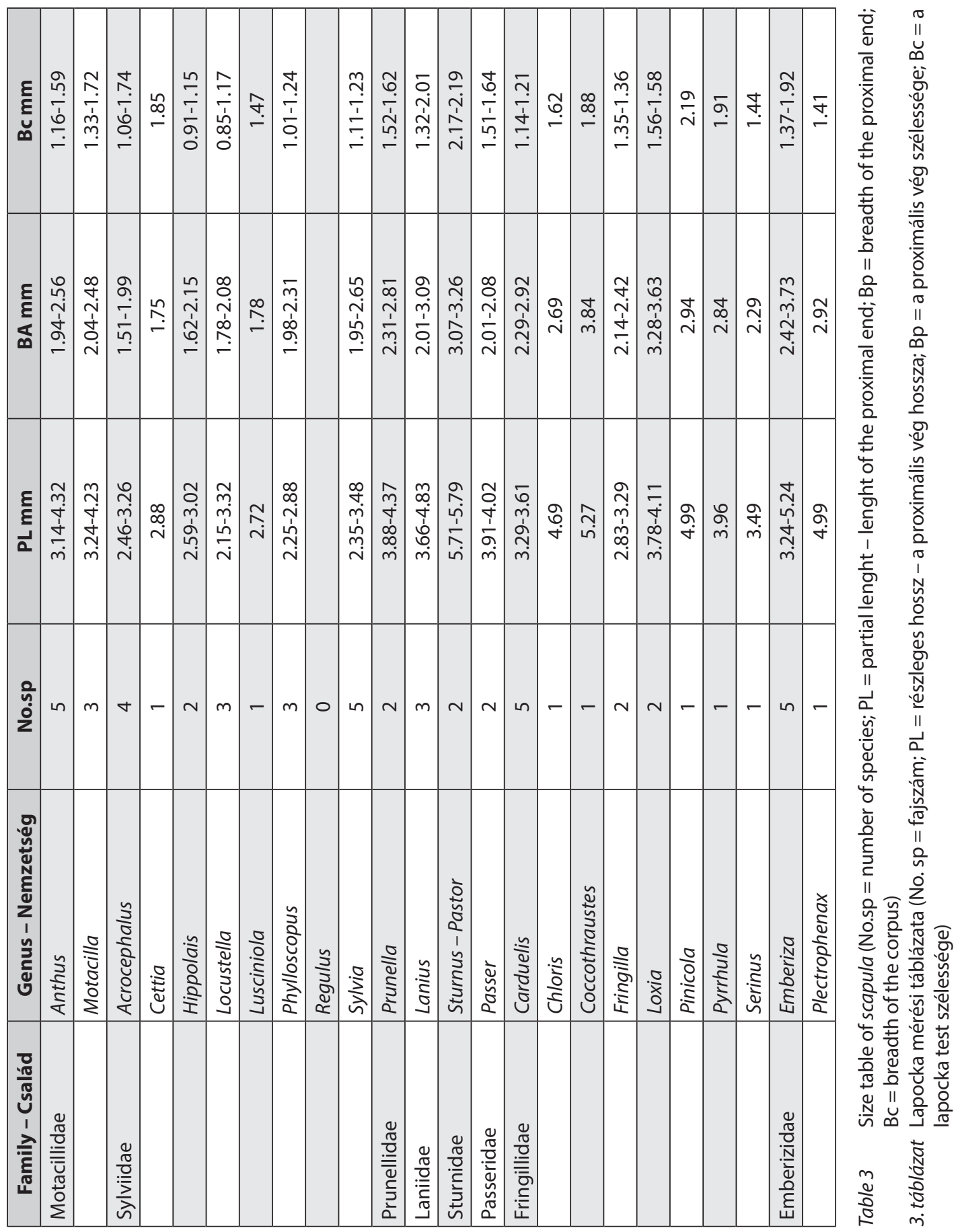




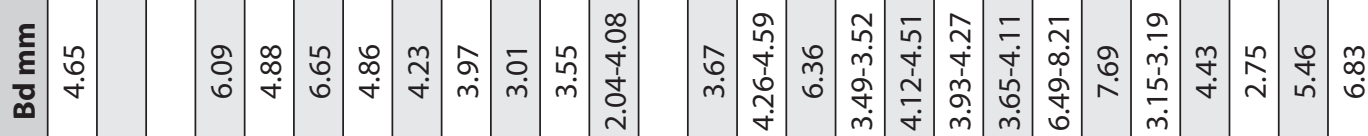

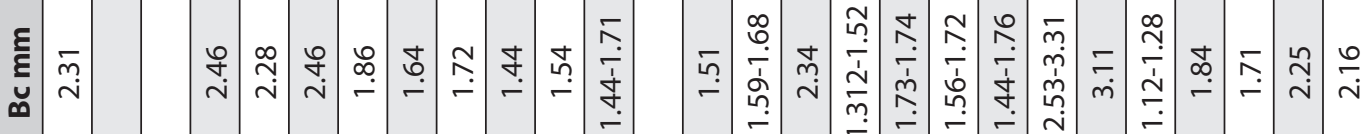

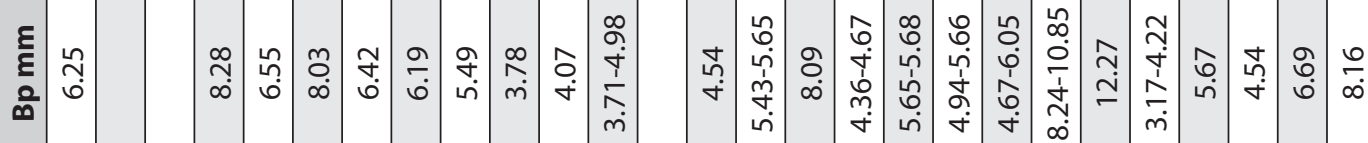

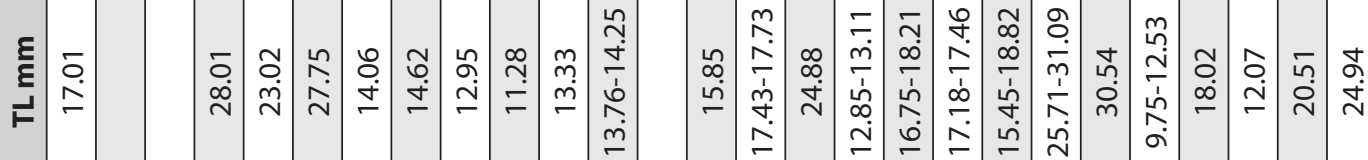

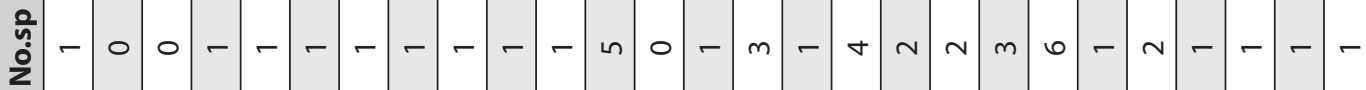

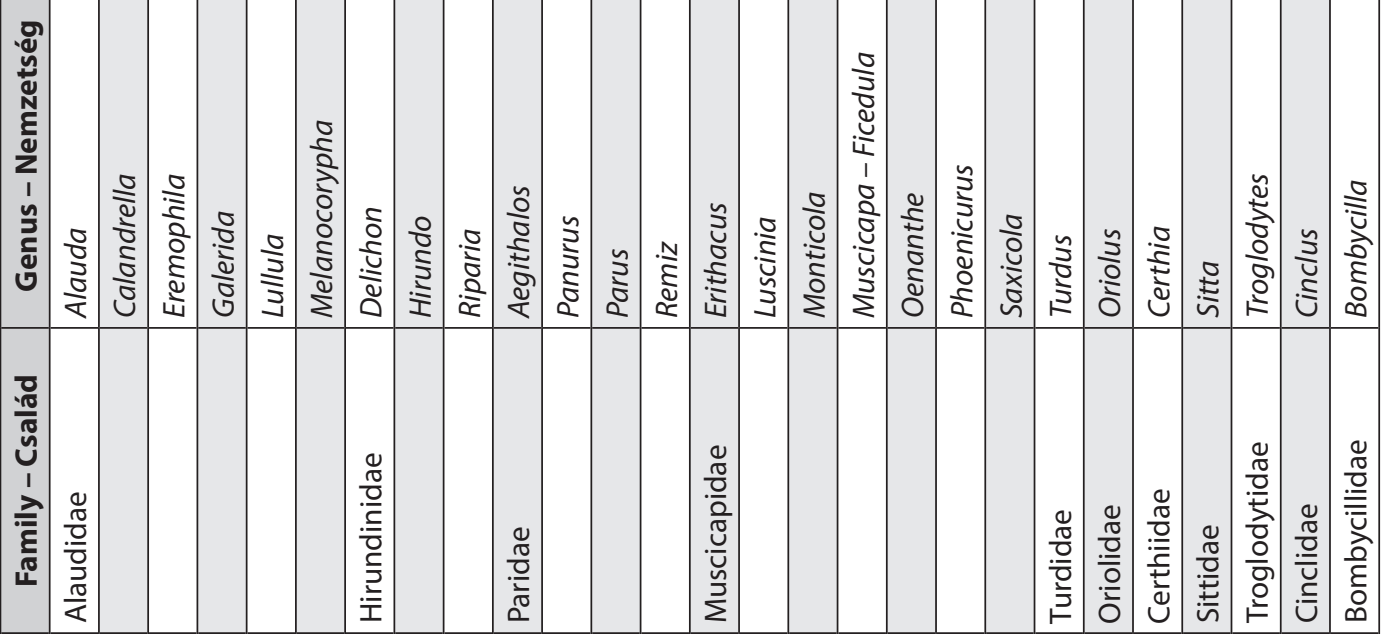




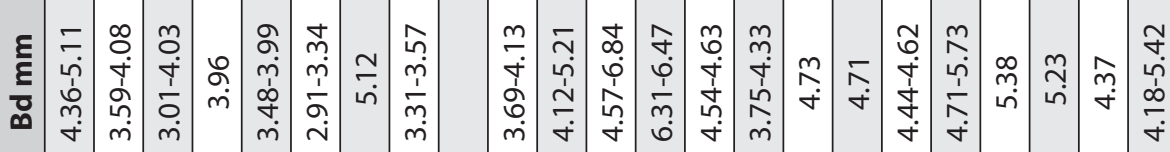

담

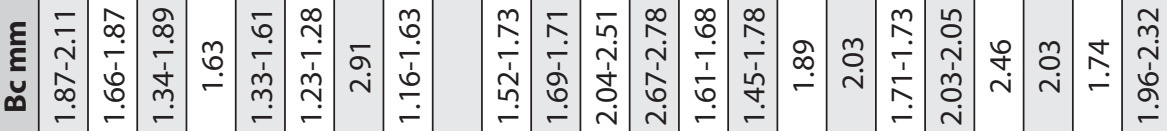

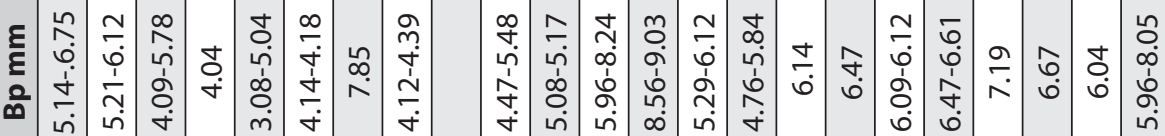

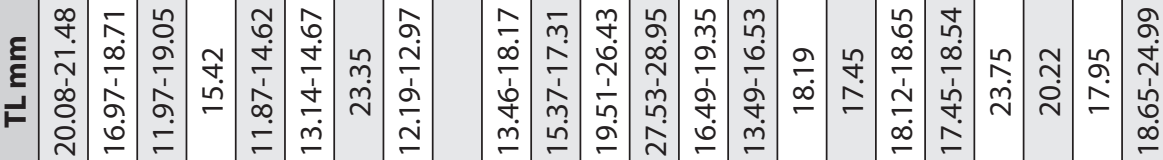

음

뭉

iั

응 으

ป ه্ঠ

‡

임

这

흔.

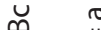

غे

बे

产

$\stackrel{\square}{\rightleftarrows}$

फ

考

ㅎํำ

응

픔

요

$\overline{\bar{T}}$

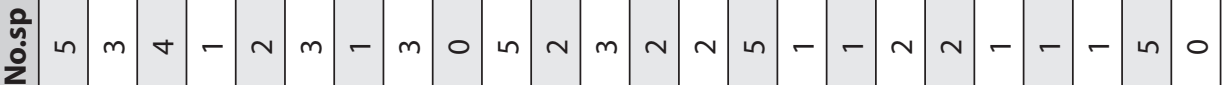

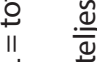

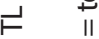

ì

¿ू.

ก N

क

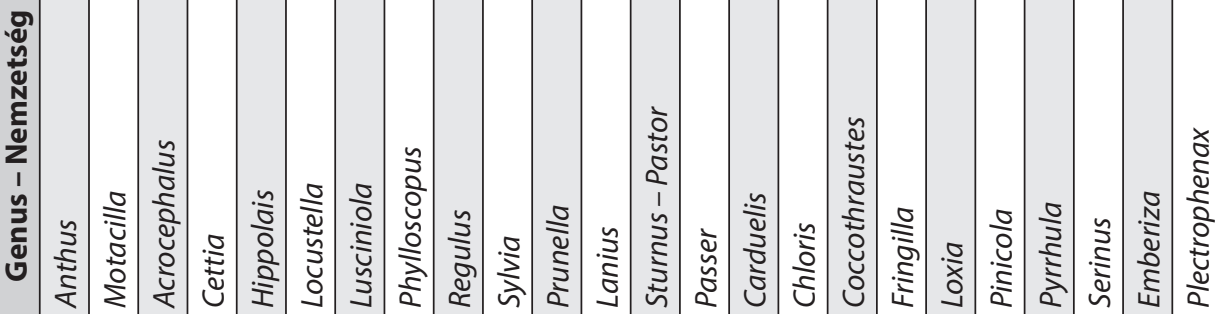

을

宅

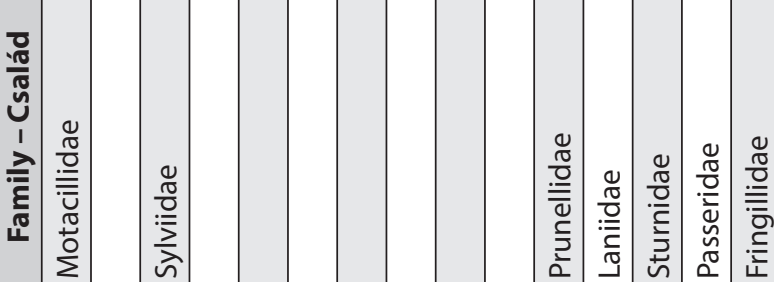

กิ

는

षे

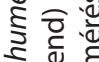

유 훙 응 웜 芷产产 


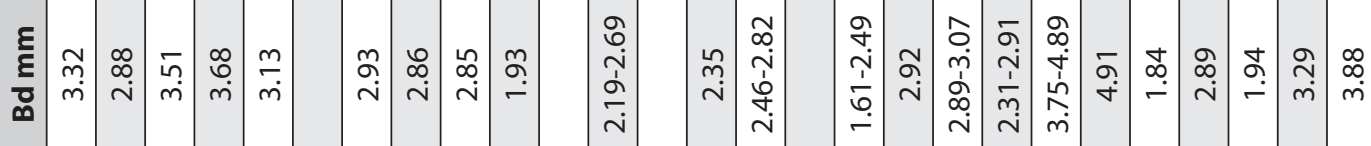

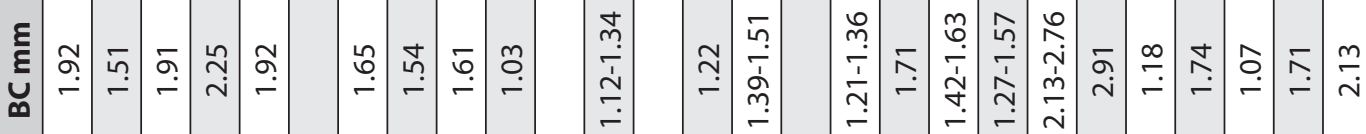

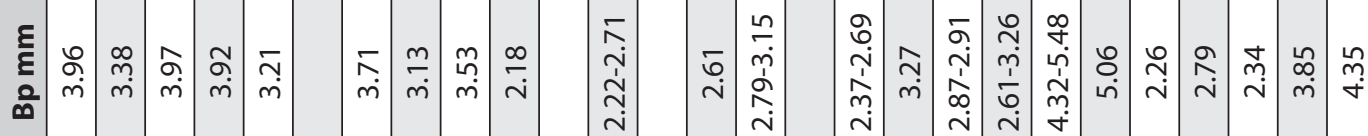

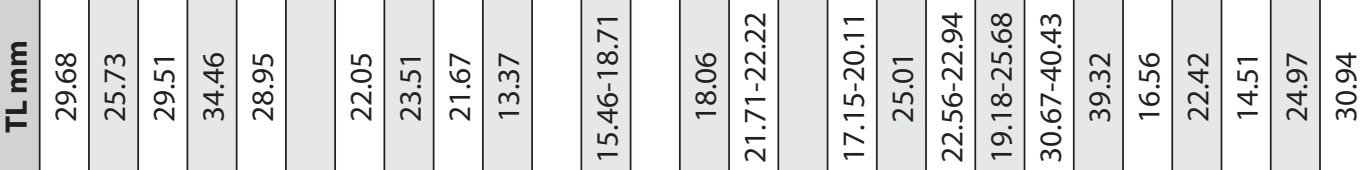
⿳亠丷厂彡

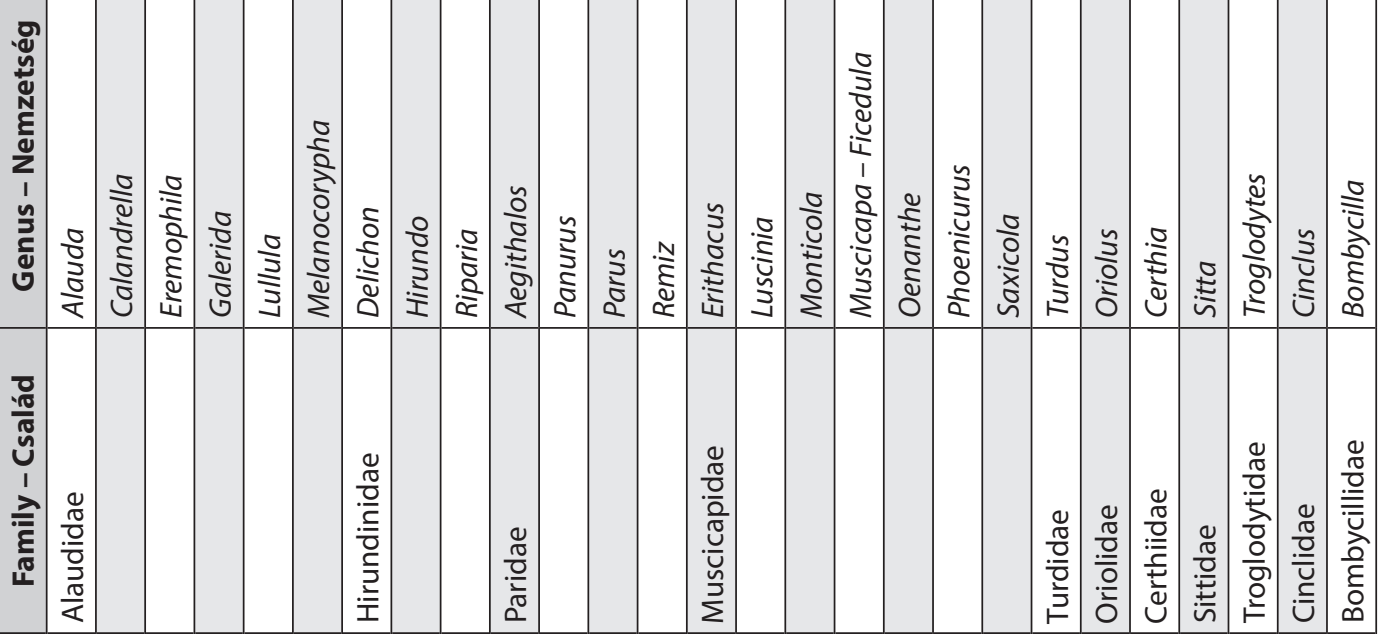




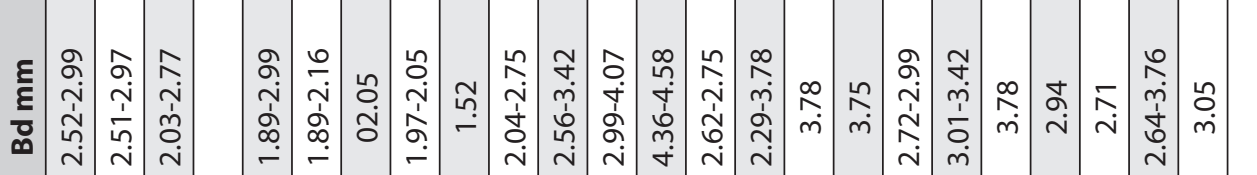

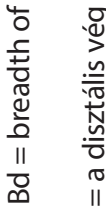

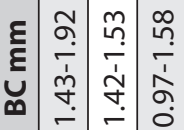

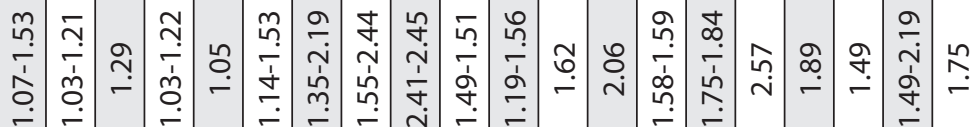

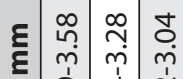

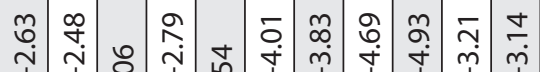

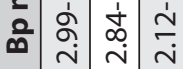

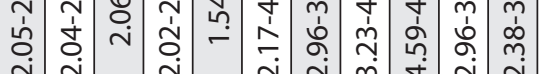

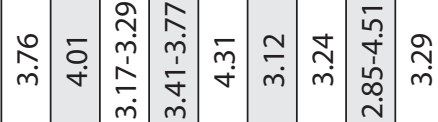

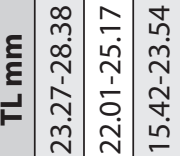

끄응

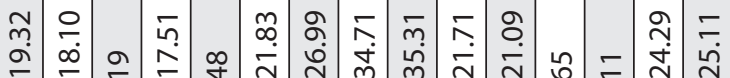

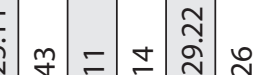

ஸ் ம்

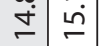

เi

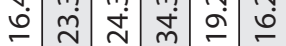

กิ

ฟู

ทั่ 드

잉 ठั

¿ जू

号

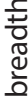

$\frac{\sqrt[n]{N}}{\frac{N}{C}}$

II II

宅

ฮ ठั่

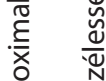

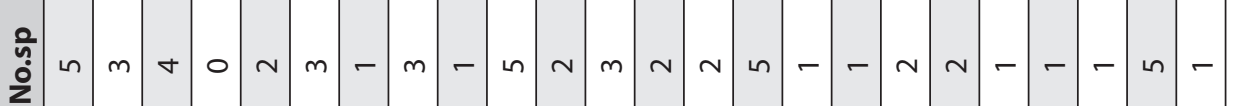

흥



范

吾

ญัต

I II

๓

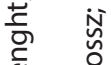

ฮ)

ग प

II

$F \quad F$

यू है

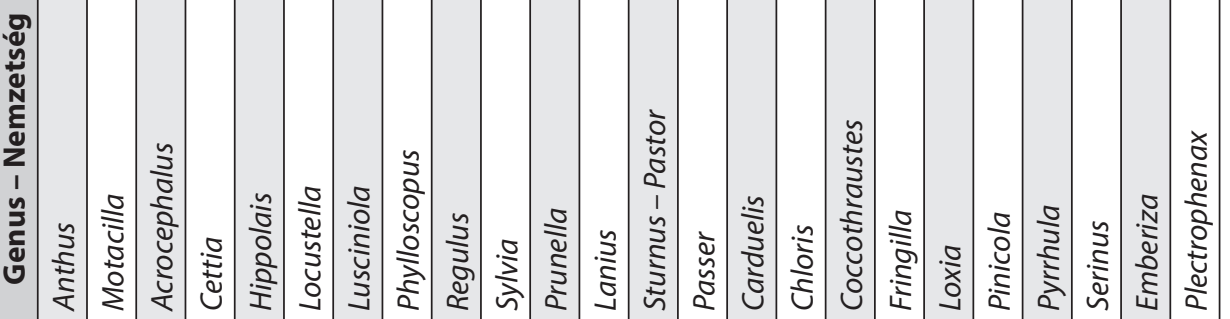

은 产

ขั ทำ

ह ํํㄹ

ᄃ

욤

은

․
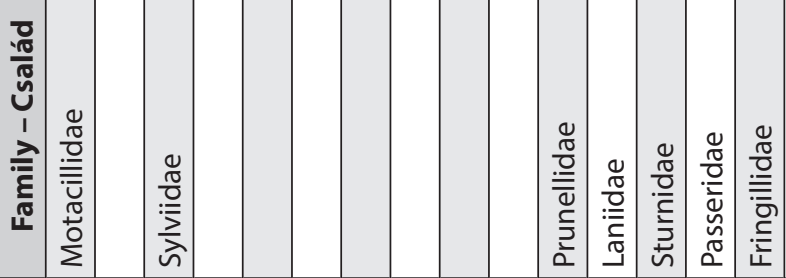

4

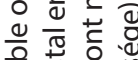

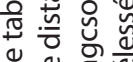

총

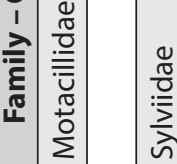

\begin{tabular}{l}
$\frac{\pi}{0}$ \\
$\frac{\pi}{0}$ \\
\hdashline$\frac{N}{2}$ \\
$\frac{0}{E}$ \\
$\frac{1}{4}$
\end{tabular}

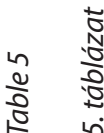




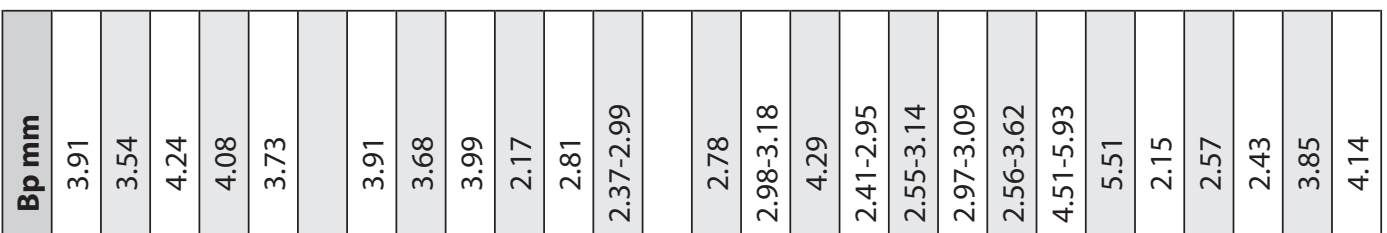

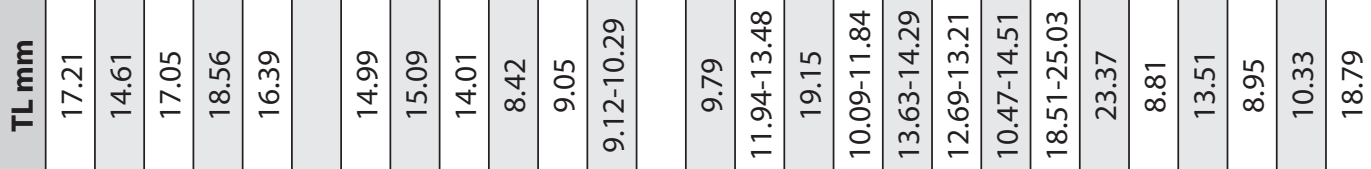
$\frac{0}{\dot{\zeta}}$

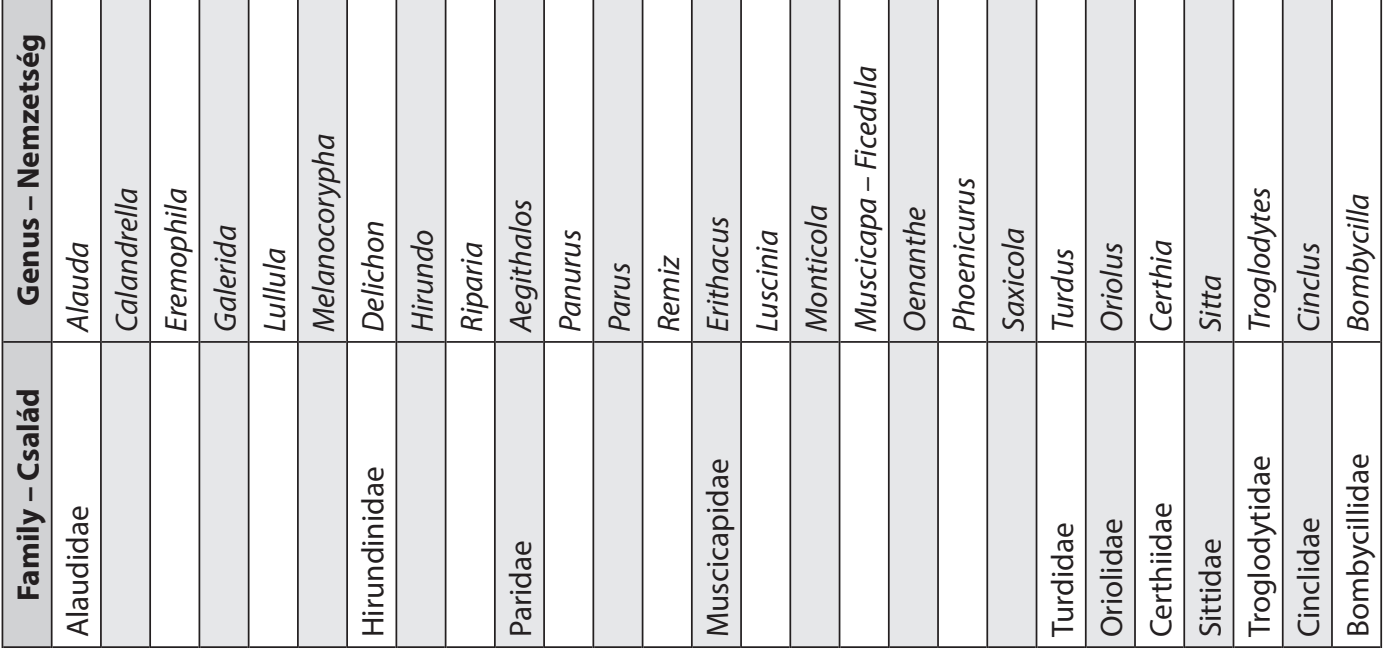




\begin{tabular}{|c|c|c|c|c|c|c|c|c|c|c|c|c|c|c|c|c|c|c|c|c|c|c|c|c|c|}
\hline $\begin{array}{l}\frac{\varepsilon}{\varepsilon} \\
\frac{\rho}{\oplus}\end{array}$ & 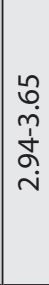 & $\begin{array}{l}m \\
0 \\
m \\
\dot{1} \\
\hat{N} \\
\dot{v}\end{array}$ & $\begin{array}{c}\bar{N} \\
m \\
\dot{\sim} \\
\infty \\
\sim\end{array}$ & & 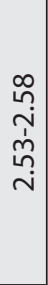 & 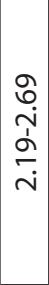 & & 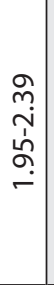 & $\begin{array}{l}\tilde{\sigma} \\
\text { i }\end{array}$ & $\begin{array}{c}m \\
m \\
m \\
\infty \\
m \\
m\end{array}$ & $\begin{array}{c}m \\
\hat{m} \\
\dot{m} \\
\tilde{m} \\
\dot{m}\end{array}$ & 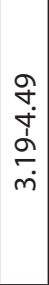 & 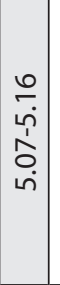 & $\begin{array}{l}0 \\
\stackrel{+}{N} \\
\text {. }\end{array}$ & 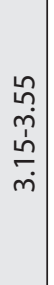 & $\begin{array}{c}\bar{f} \\
m\end{array}$ & $\bar{E}$ & $\begin{array}{l}\dot{J} \\
m\end{array}$ & $\begin{array}{l}\infty \\
0 \\
\dot{+} \\
\dot{1} \\
\dot{ர} \\
\dot{m}\end{array}$ & $\stackrel{\stackrel{n}{m}}{\forall}$ & $\begin{array}{l}\stackrel{0}{0} \\
\dot{m}\end{array}$ & $\stackrel{\stackrel{\sim}{m}}{m}$ & 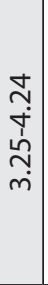 & $\begin{array}{l}\stackrel{q}{+} \\
\dot{m}\end{array}$ & 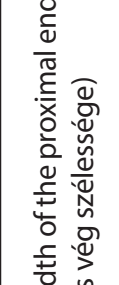 \\
\hline $\begin{array}{l}\underline{\varepsilon} \\
\underline{\varepsilon} \\
\vec{F}\end{array}$ & 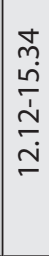 & 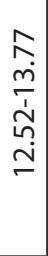 & 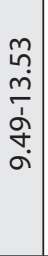 & & 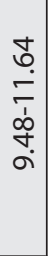 & 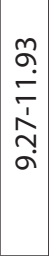 & & $\begin{array}{l}\hat{n} \\
0 \\
\frac{1}{12} \\
\sigma \\
\sigma\end{array}$ & $\underset{\bar{\sigma}}{\sim}$ & 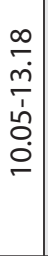 & 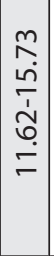 & 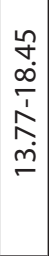 & 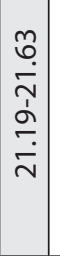 & 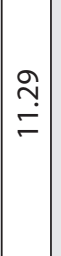 & 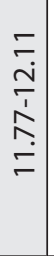 & \begin{tabular}{|c|}
$\stackrel{m}{ }$ \\
$\infty$ \\
$\dot{m}$ \\
-
\end{tabular} & $\begin{array}{l}m \\
\stackrel{n}{n} \\
\end{array}$ & $\begin{array}{l}\stackrel{\sim}{\tilde{Z}} \\
\stackrel{+}{\leftarrow}\end{array}$ & 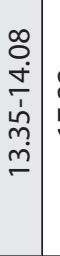 & $\begin{array}{l}\tilde{n} \\
\end{array}$ & $\begin{array}{l}\bar{ָ} \\
\stackrel{+}{ \pm}\end{array}$ & 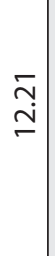 & 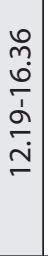 & 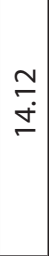 & 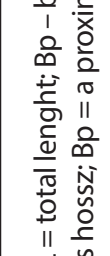 \\
\hline $\begin{array}{l}\text { जิ } \\
\text { o } \\
2\end{array}$ & in & $m$ & $\nabla$ & 0 & $N$ & $m$ & 0 & $m$ & - & in & $\sim$ & $m$ & $N$ & - & in & - & - & - & $\sim$ & - & - & - & $\nabla$ & - & 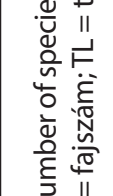 \\
\hline 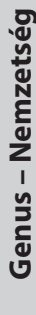 & $\frac{n}{\Sigma}$ & 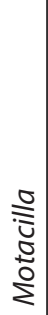 & 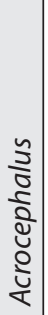 & 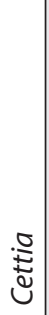 & $\begin{array}{l}\frac{n}{0} \\
\frac{0}{0} \\
\frac{a}{2} \\
\frac{1}{2}\end{array}$ & 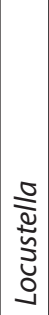 & 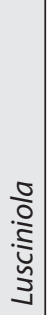 & 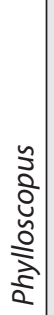 & 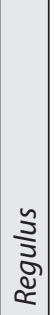 & $\frac{.0}{2}$ & $\mid \begin{array}{c}\frac{0}{2} \\
\frac{2}{2} \\
\frac{2}{2} \\
\end{array}$ & 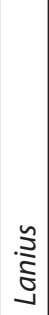 & 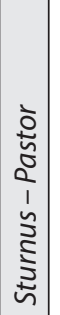 & $\begin{array}{l}\bar{\Xi} \\
\tilde{\breve{\sigma}} \\
\Sigma\end{array}$ & $\begin{array}{l}\approx \\
\frac{a}{3} \\
\frac{0}{0} \\
u\end{array}$ & 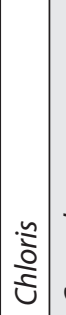 & 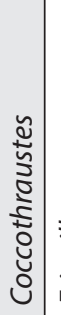 & $\stackrel{0}{\stackrel{5}{5}}$ & 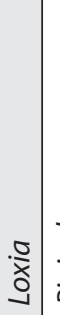 & $\stackrel{0}{0}$ & 齐 & 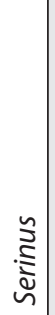 & $\begin{array}{l}\stackrel{.}{N} \\
\bar{\Xi} \\
\text { है } \\
\text { w }\end{array}$ & 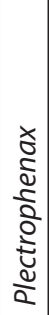 & 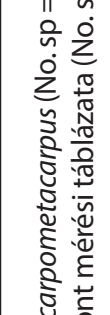 \\
\hline 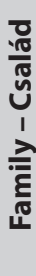 & 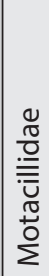 & & $\begin{array}{l}\frac{0}{\pi} \\
\frac{\pi}{0} \\
: \frac{2}{\lambda} \\
\text { n }\end{array}$ & & & & & & & & 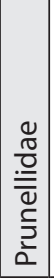 & 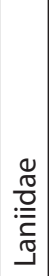 & 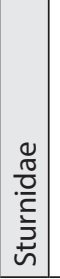 & 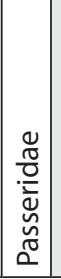 & 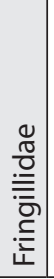 & & & & & & & & $\begin{array}{l}\frac{0}{0} \\
\frac{0}{N} \\
\frac{N}{2} \\
\frac{0}{E} \\
\frac{5}{\omega}\end{array}$ & & 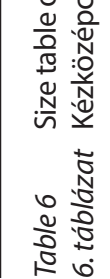 \\
\hline
\end{tabular}




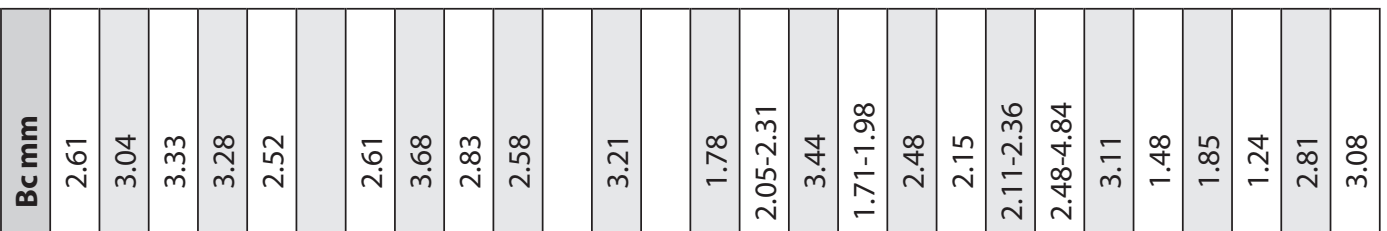

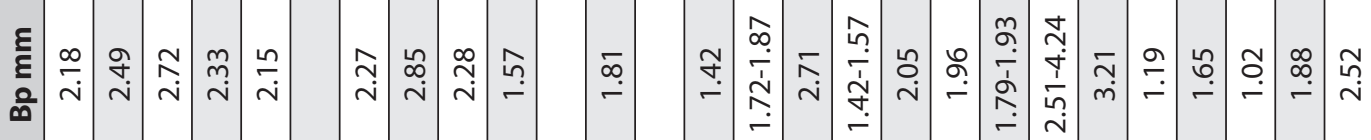

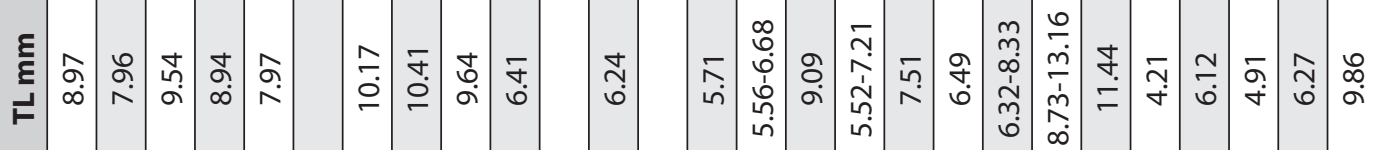
$\frac{0}{\dot{0}}$

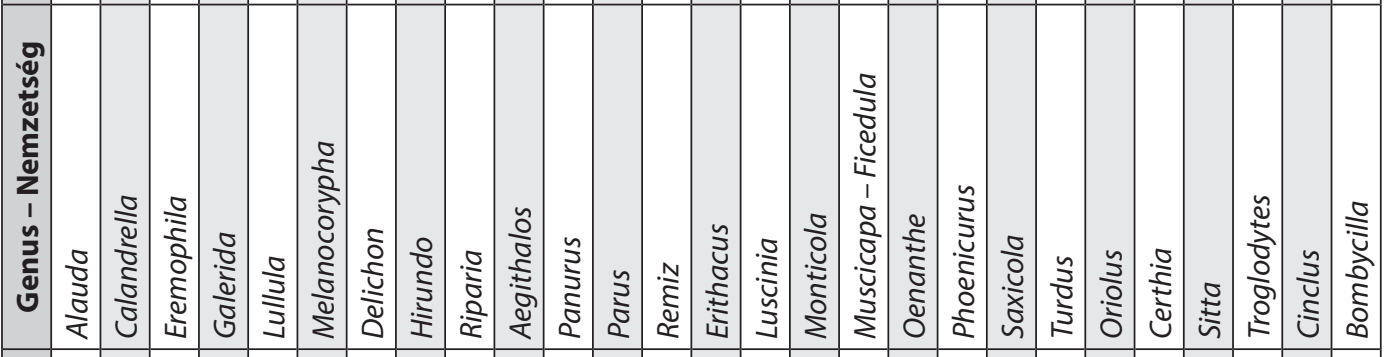

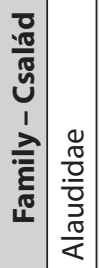

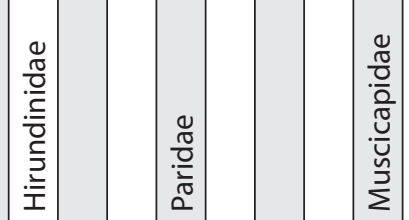

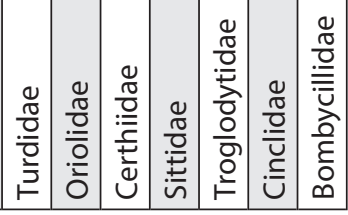




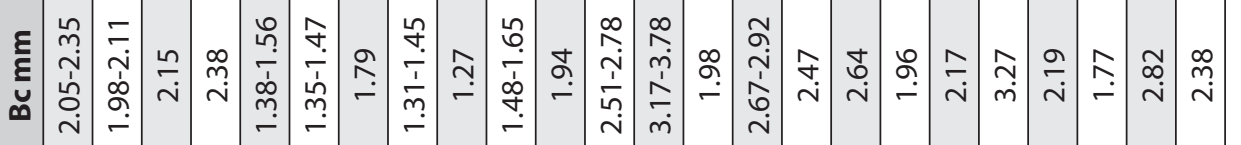

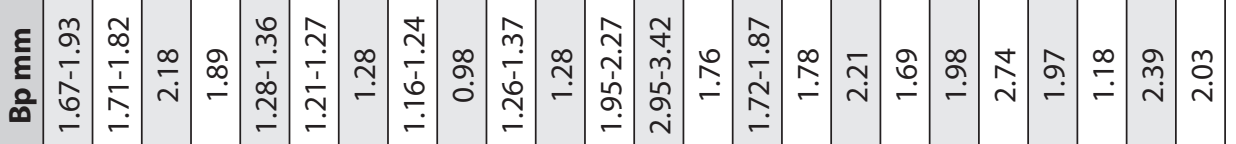

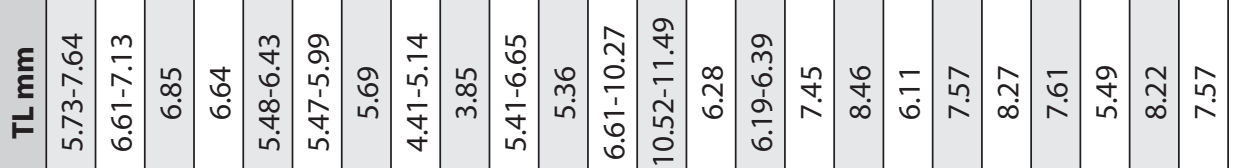

ㅎํㅁ

II

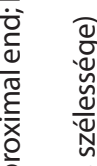

을

竞

吾 II

ฐ্ّ

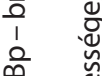

吾

ब

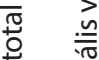

11. है

$\vec{F}$

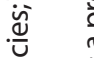

요

in

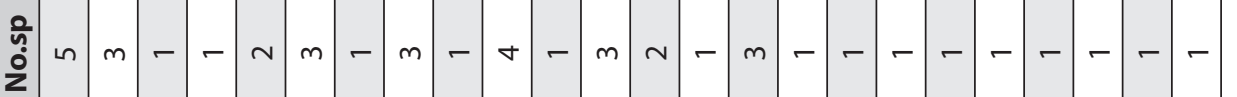

离

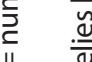

की II

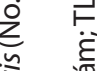

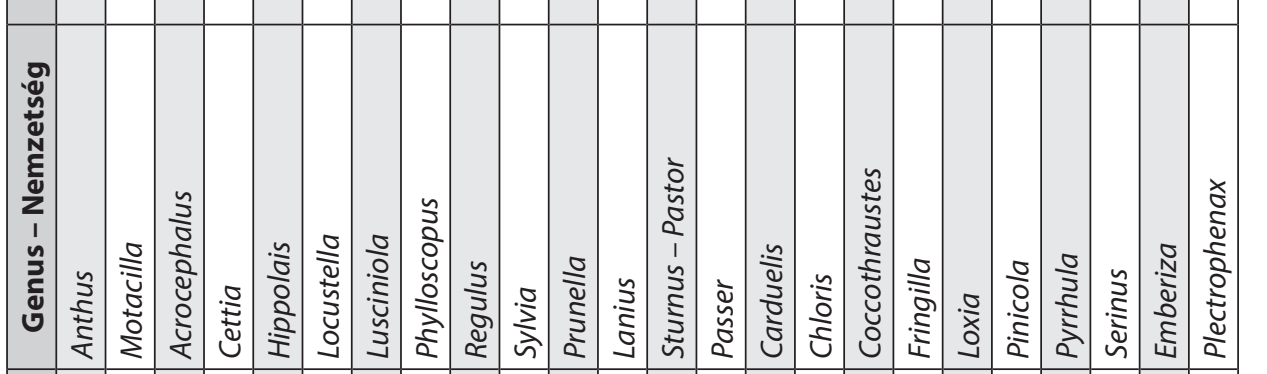

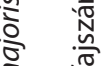

ह 11

하

ㄴ. प

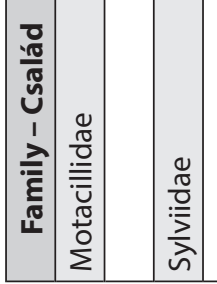

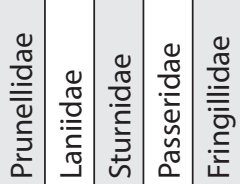




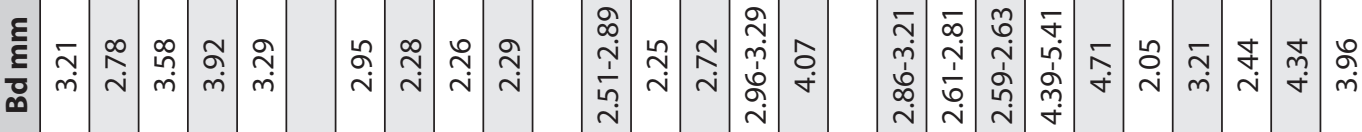

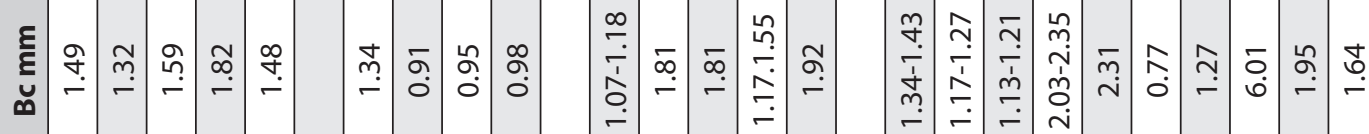

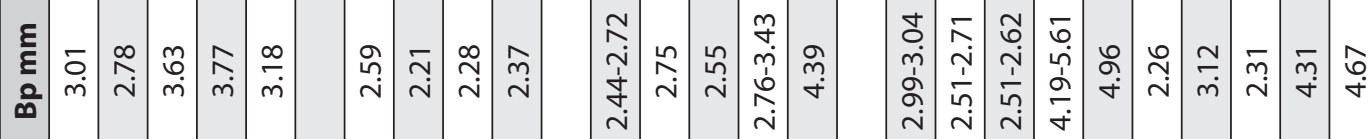

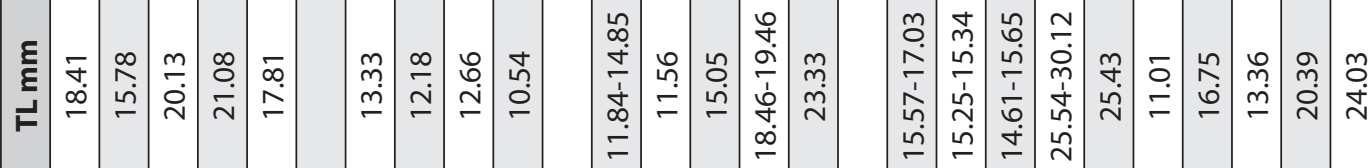

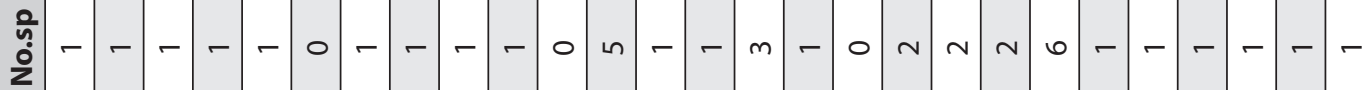

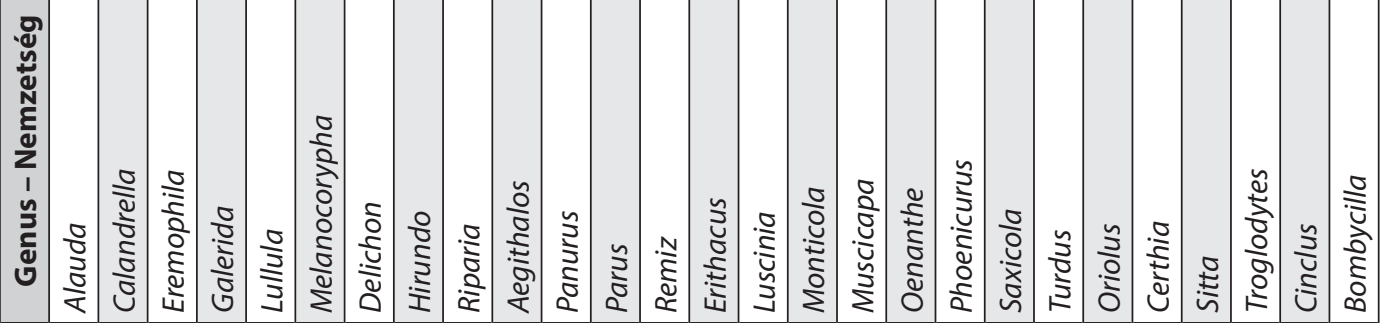

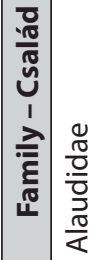




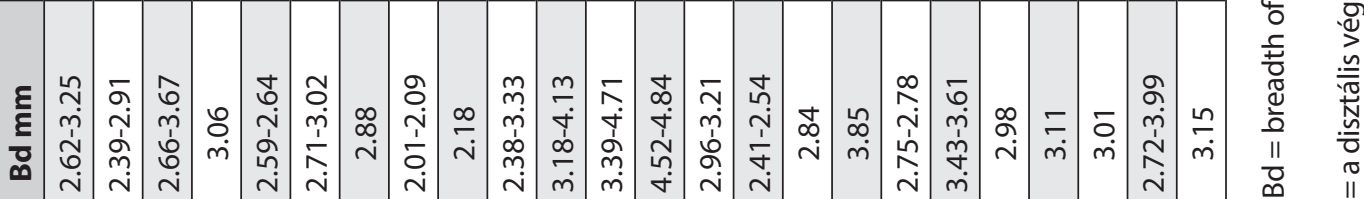

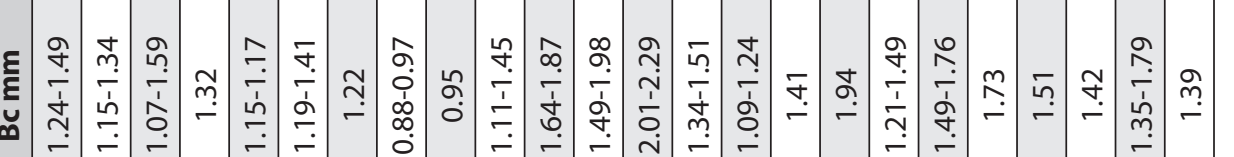

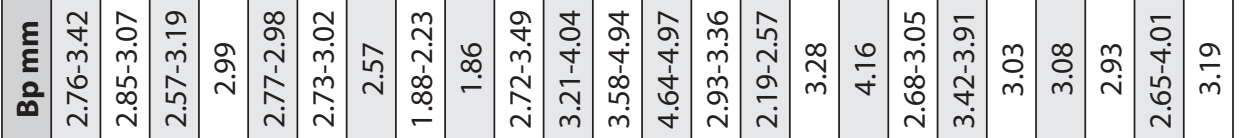

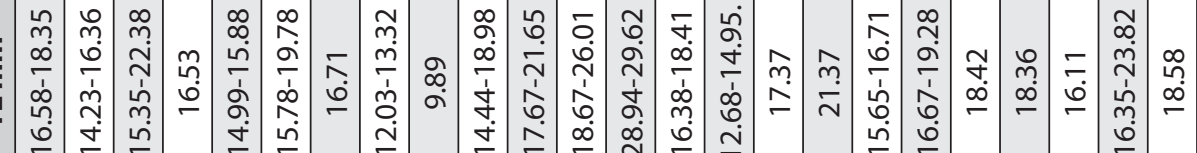

ทั่ 므

过

‡

章

迹

II

○ u

ซ் dิ

ֻั0

¿

to

吾

음

을

으 음

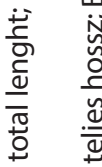

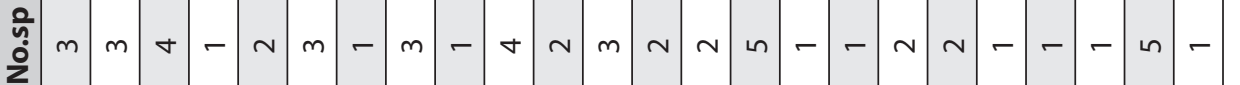

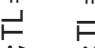

这

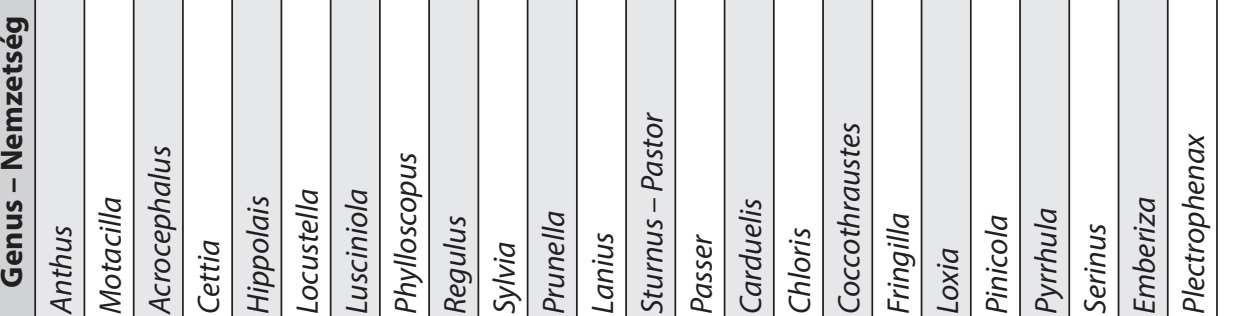

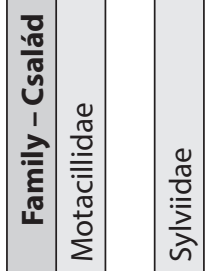

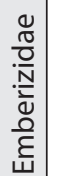

范

के

है ڤำ

II $\quad$ 苞

กํ. 눙

ह

है

웡 ฮ

응 覀

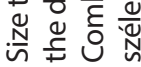

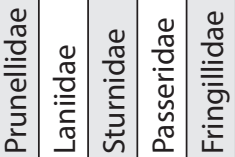

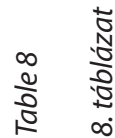




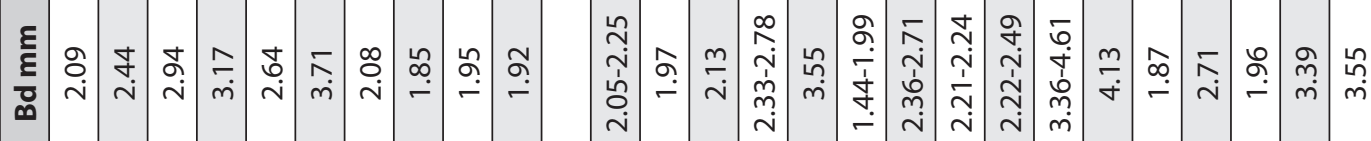

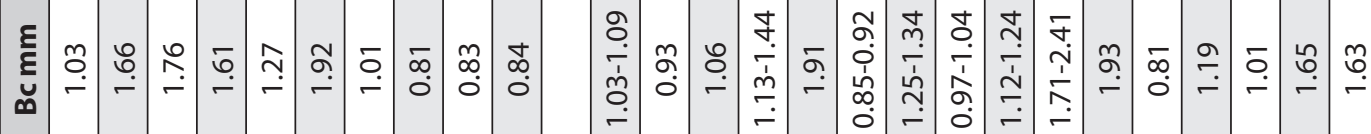

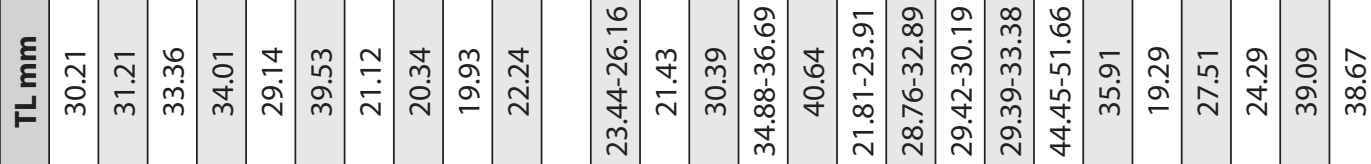

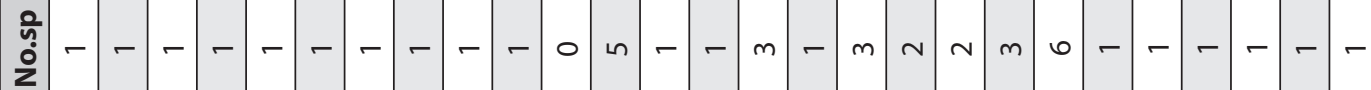

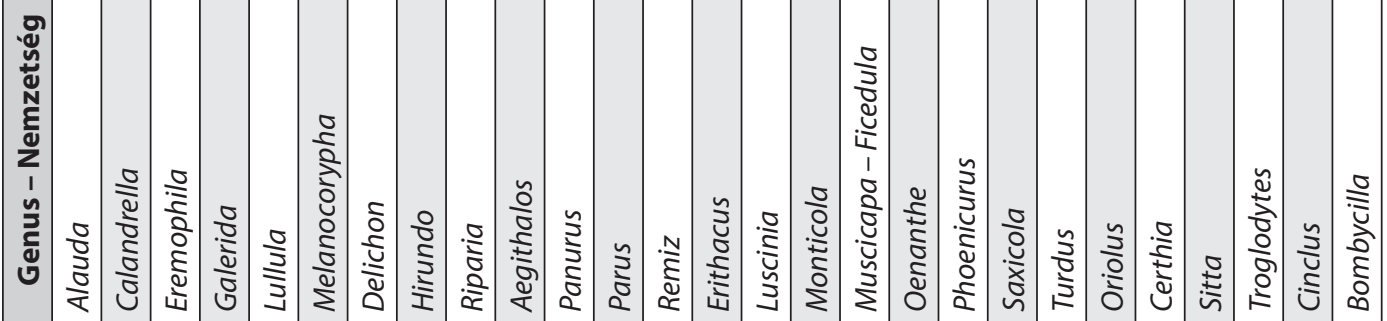

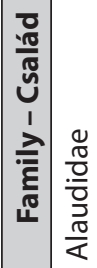

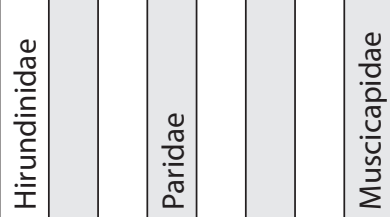

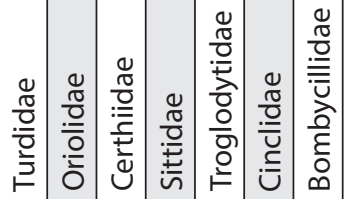




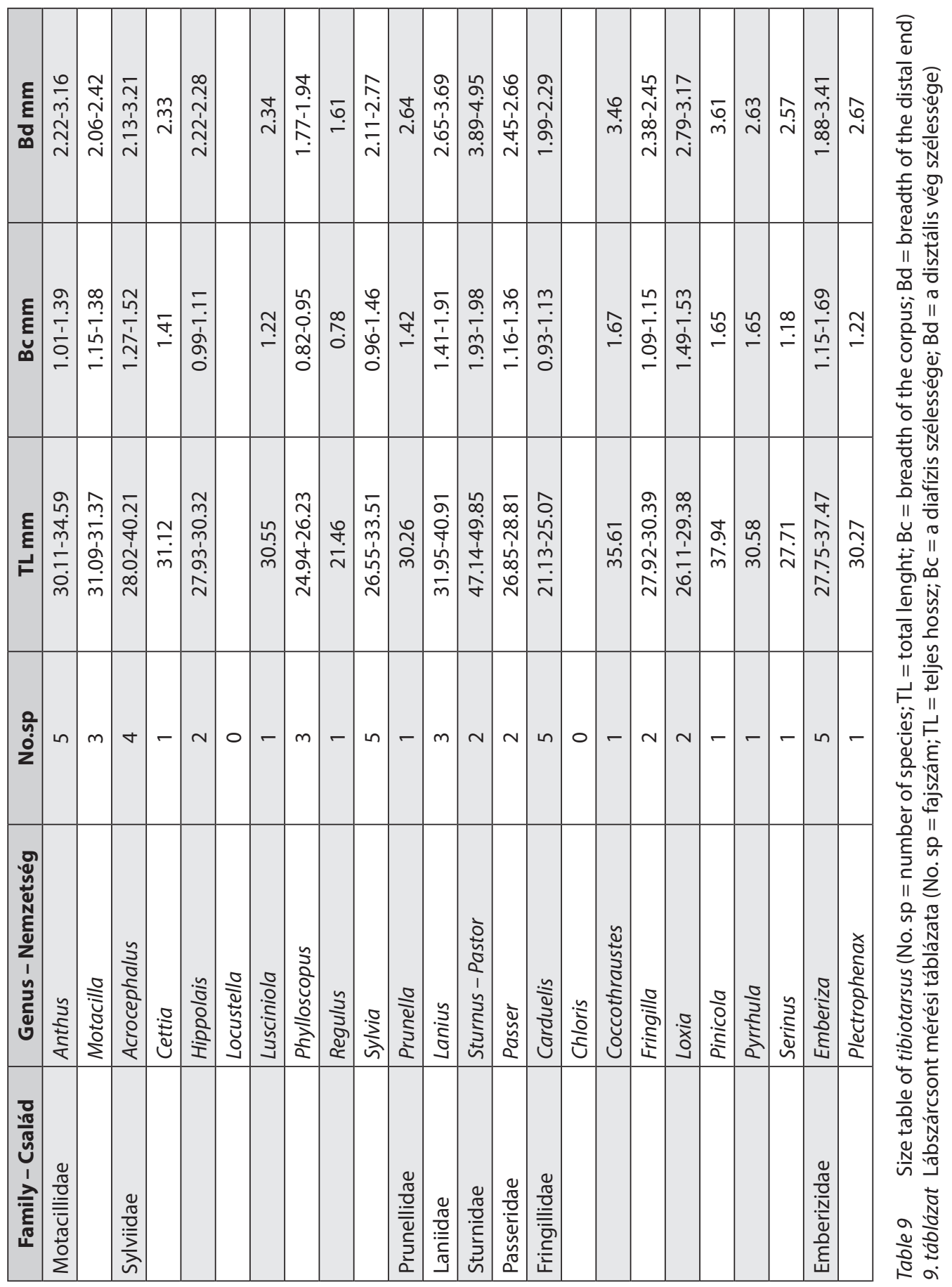




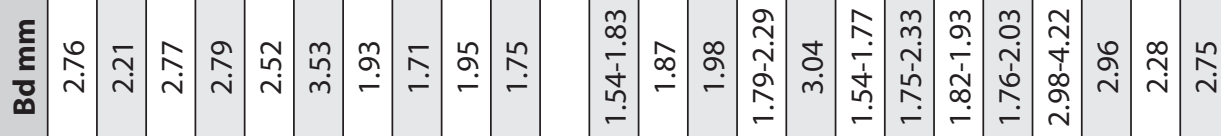

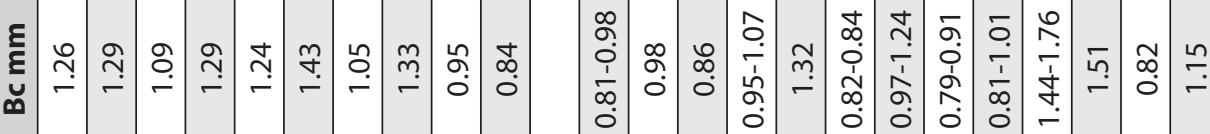

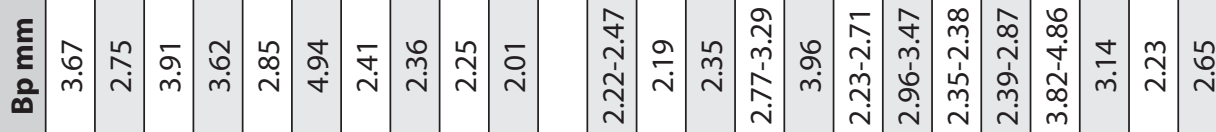

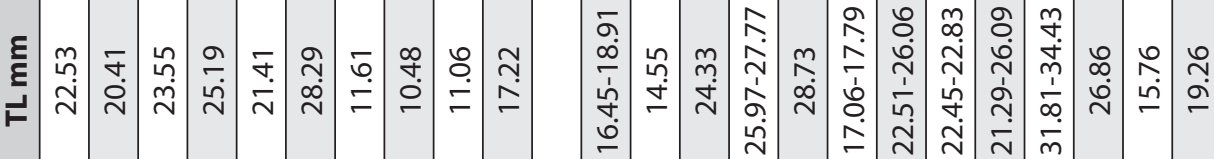

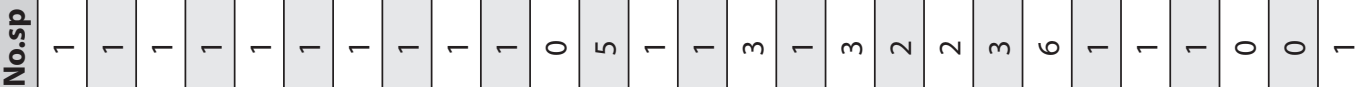

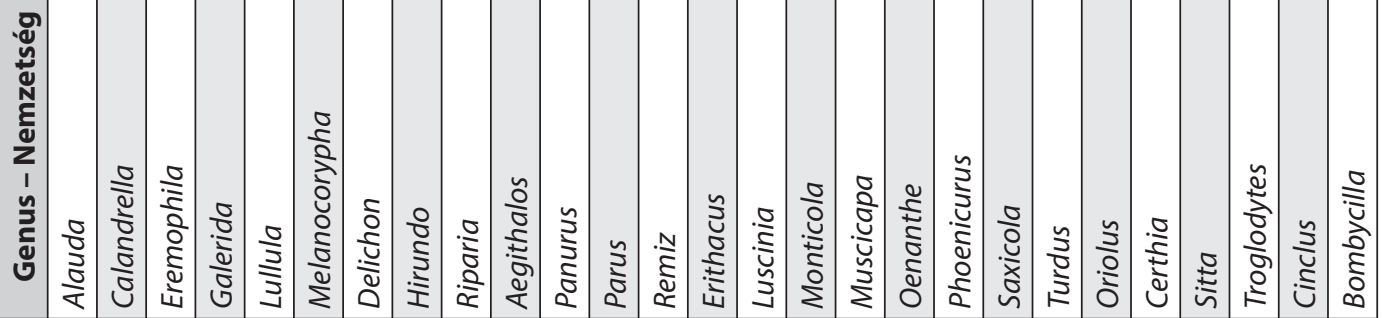

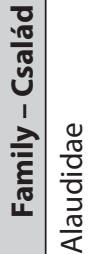

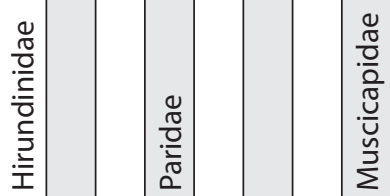

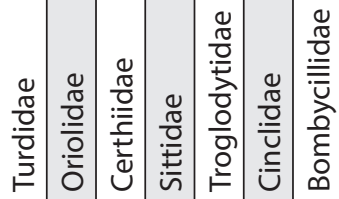




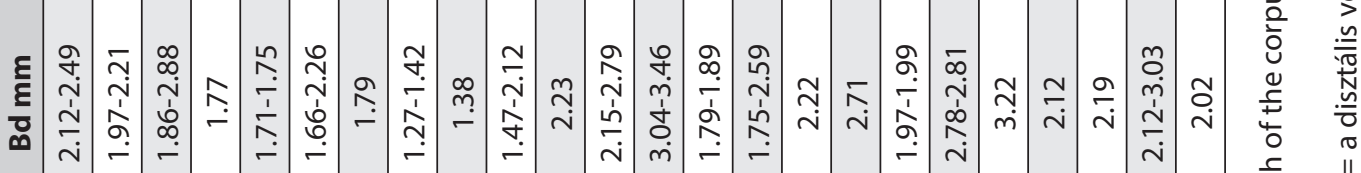

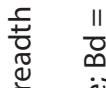

E

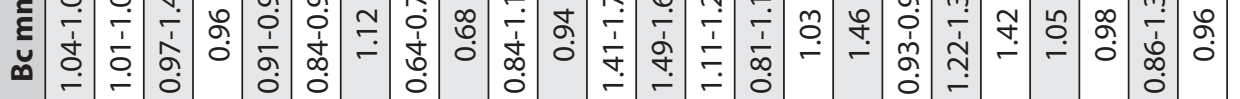

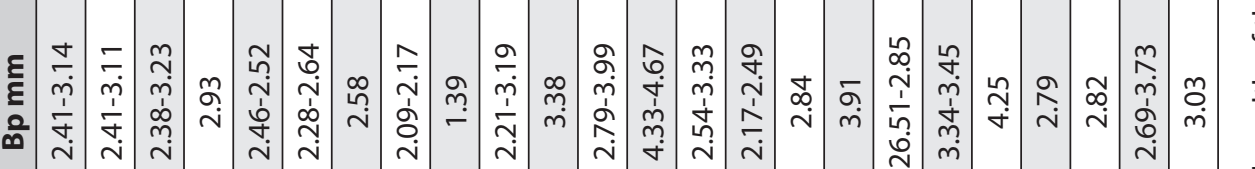

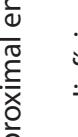

임

$\check{\varnothing}$

m.

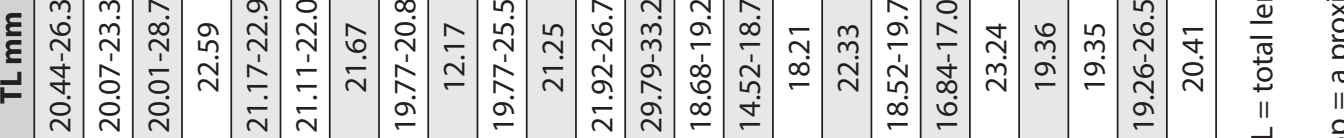

$\frac{0}{0}$
:

$\stackrel{0}{*}$ ஜ

iั N

这

$\stackrel{\tilde{c}}{\stackrel{n}{*}}$

¿

$\stackrel{\vec{E}}{\rightleftarrows}$

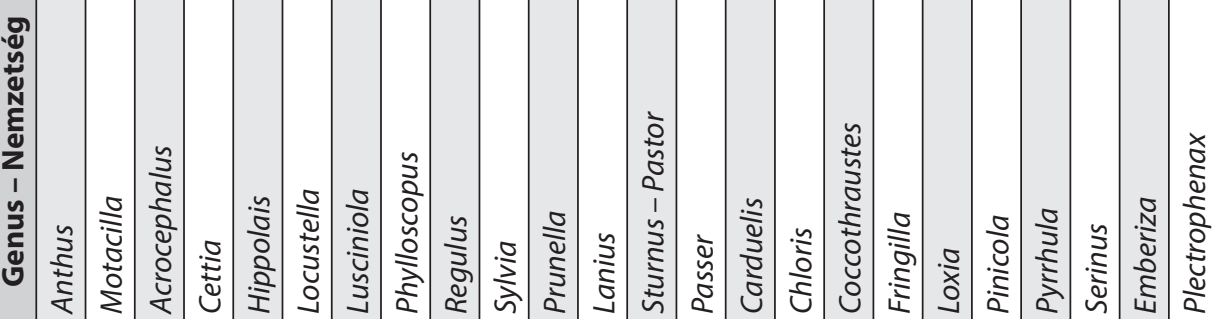

॥

은 $\frac{N}{\pi}$

으 임

ㅎํ 우

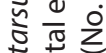

응

है N

ปัँ

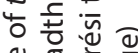

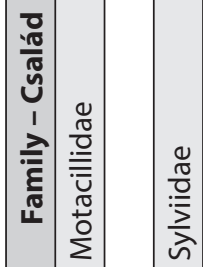

造

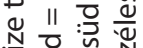

动

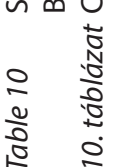




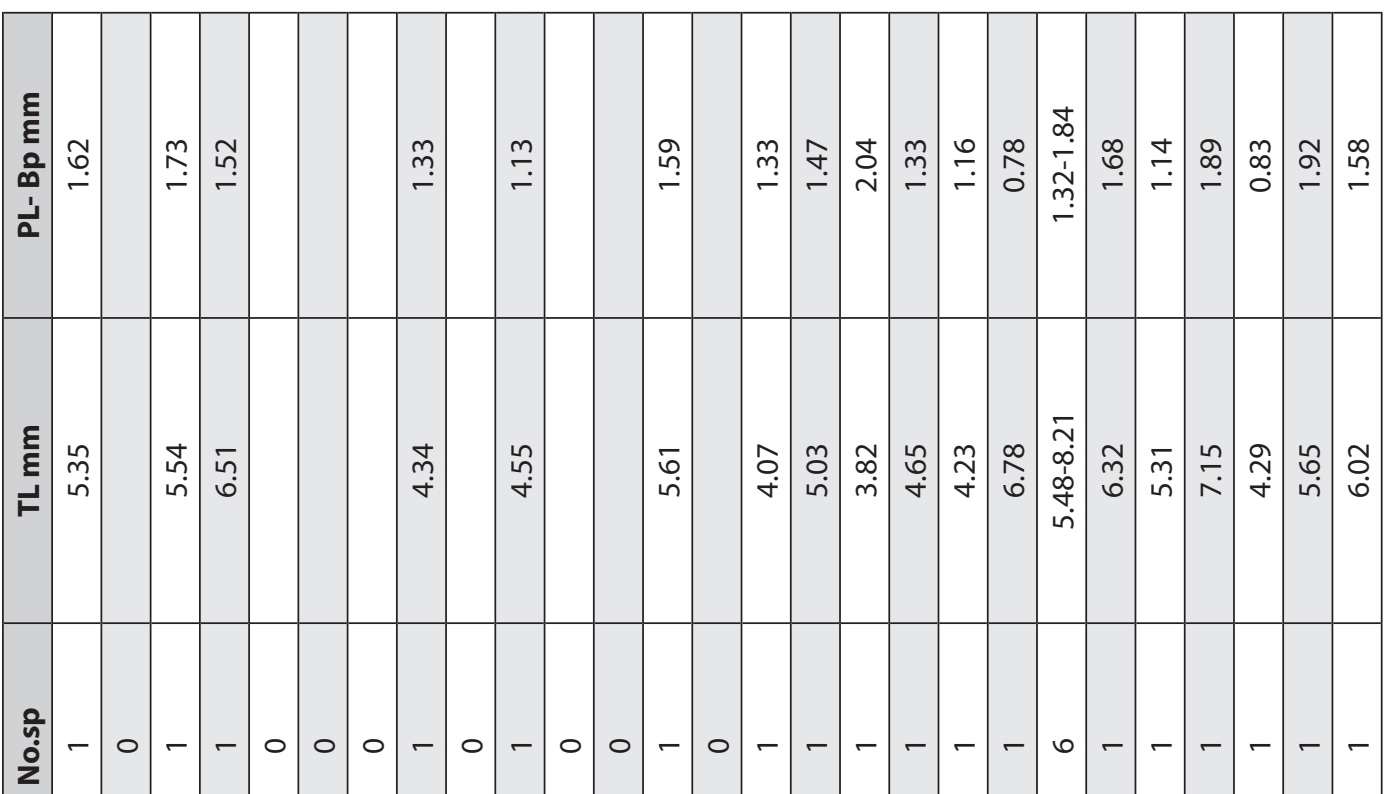

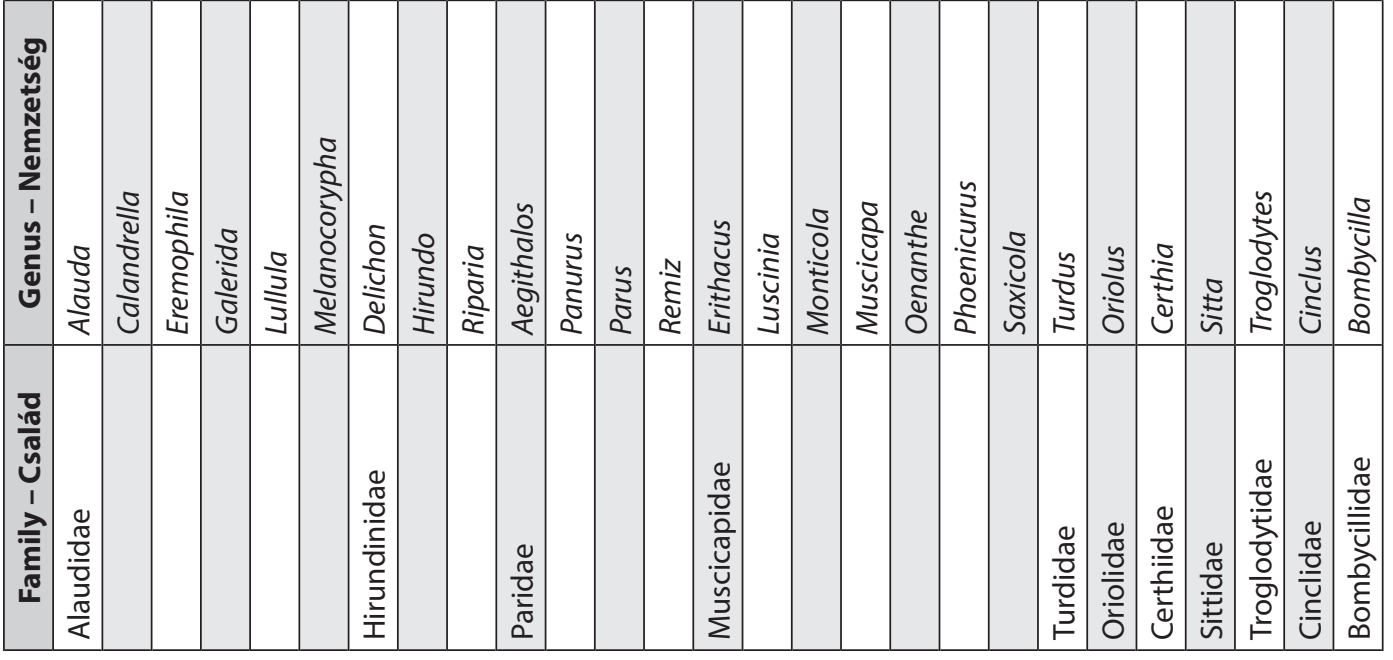




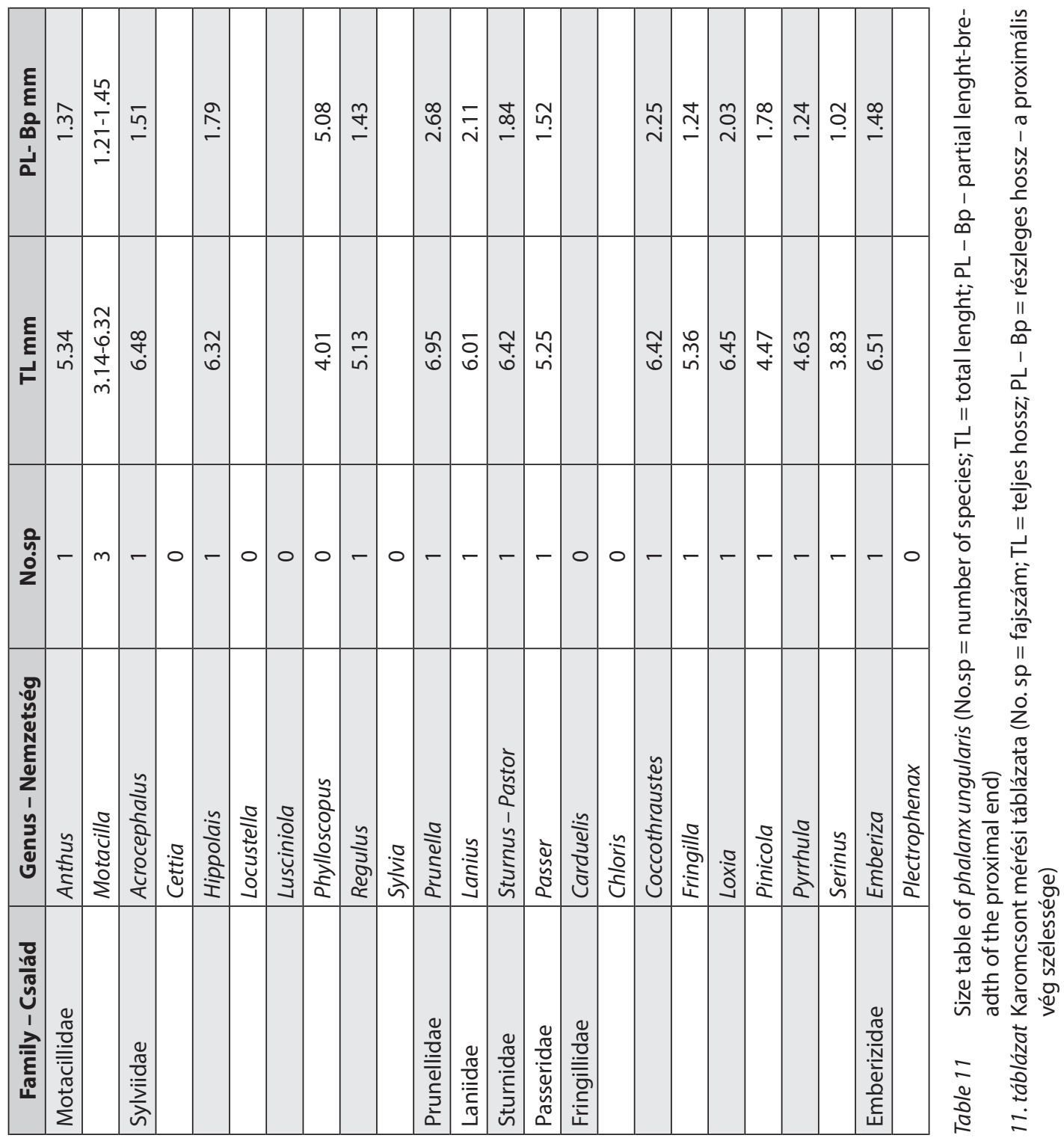



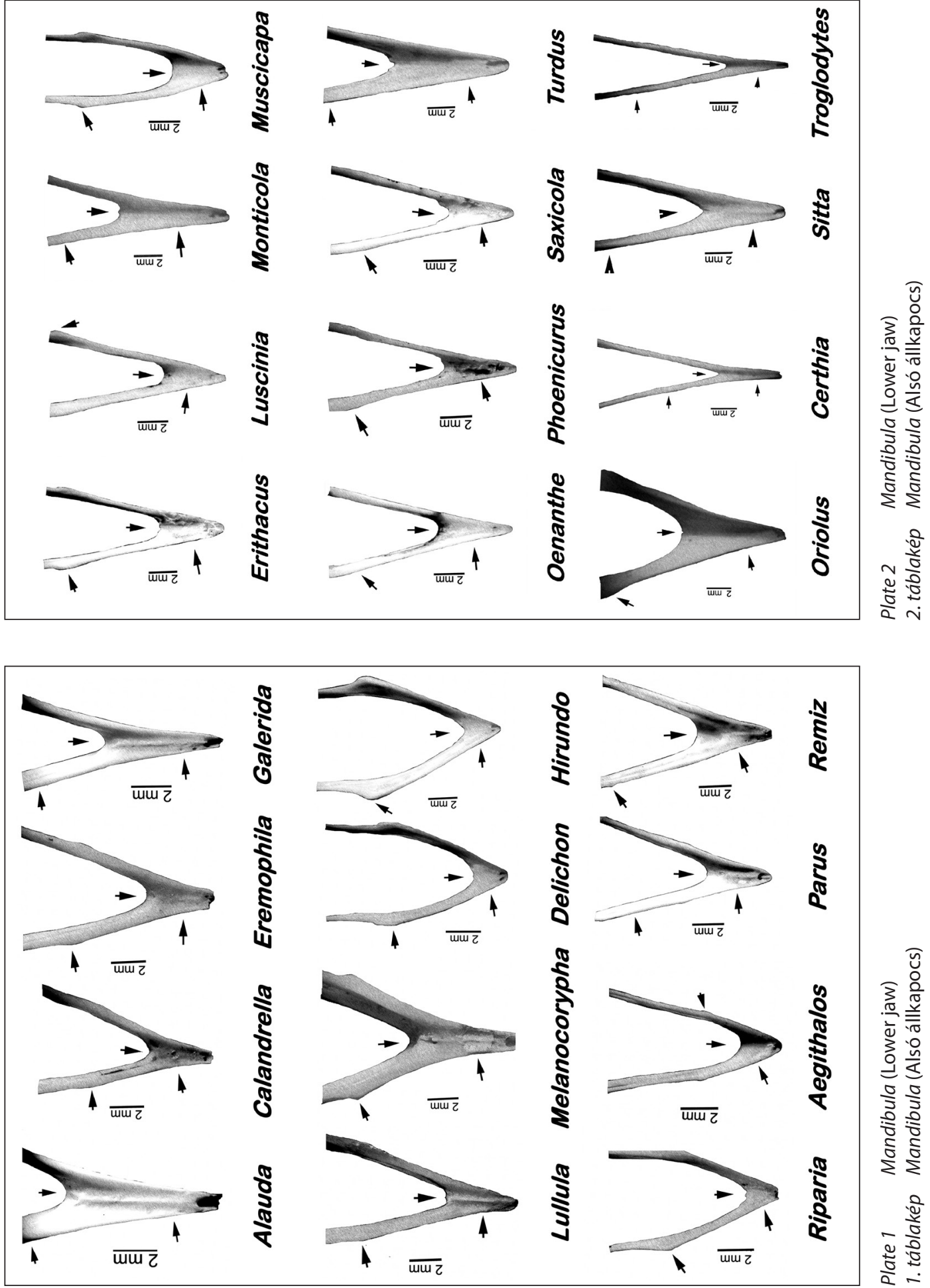


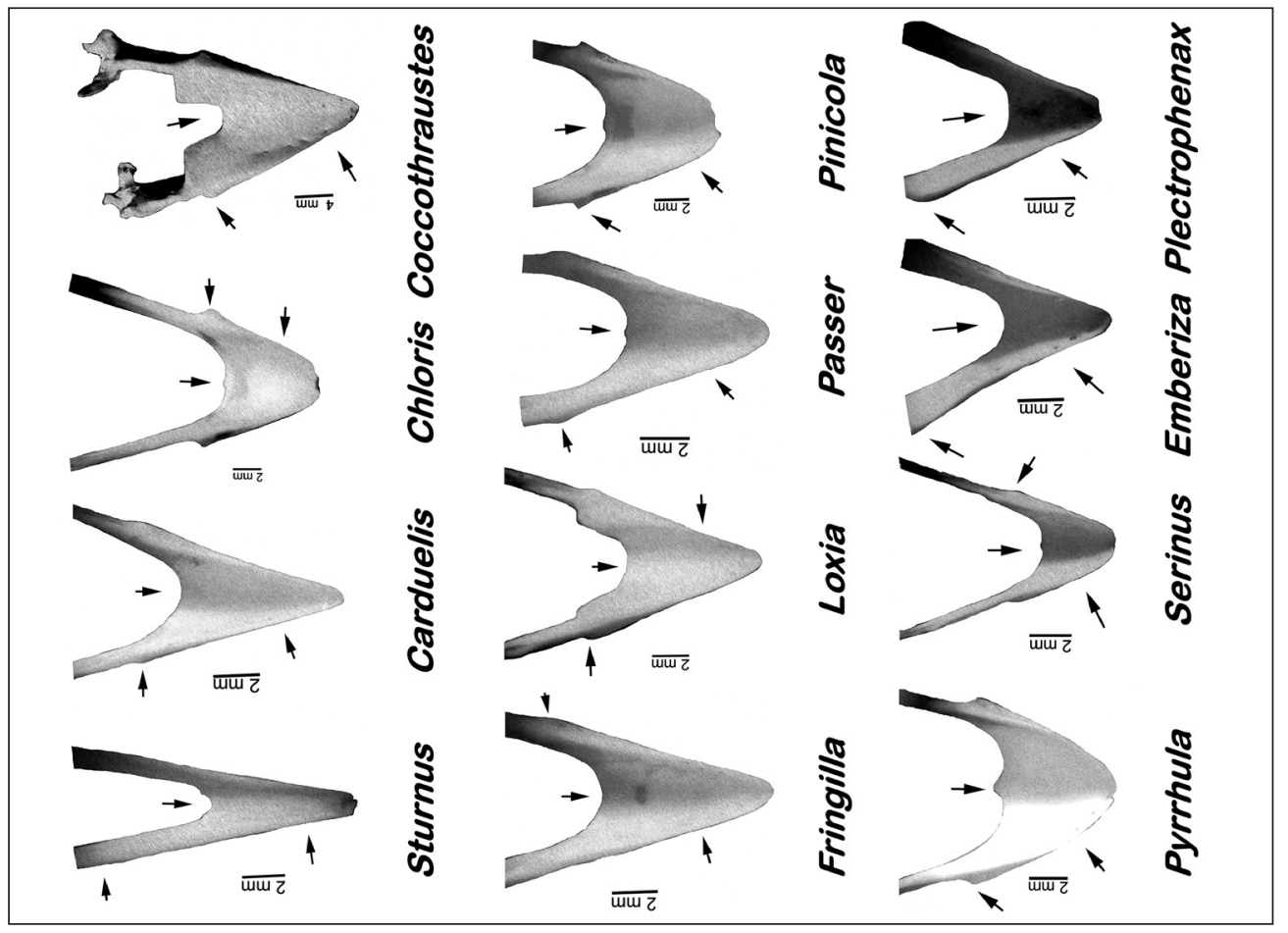

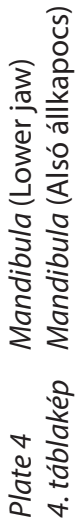

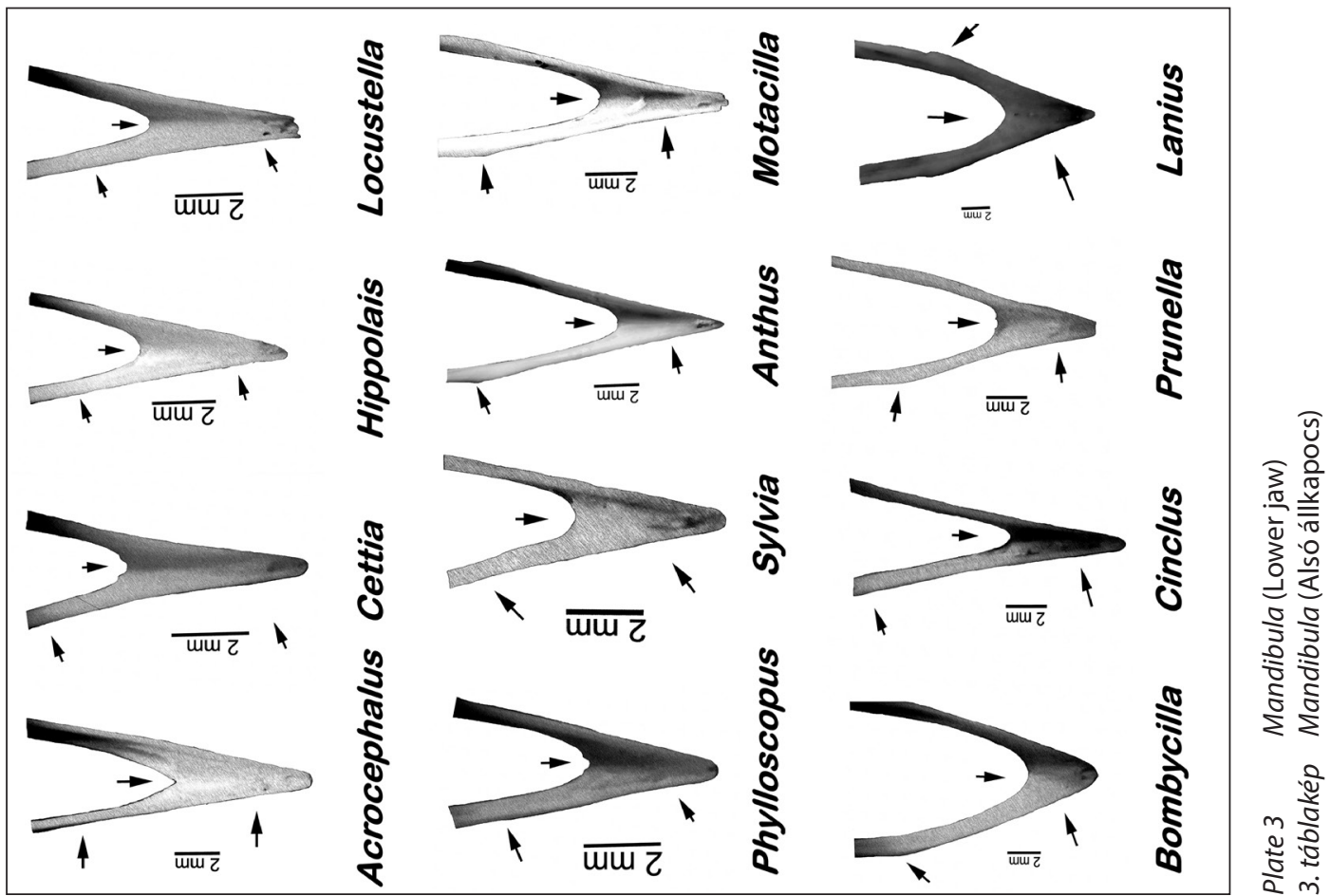



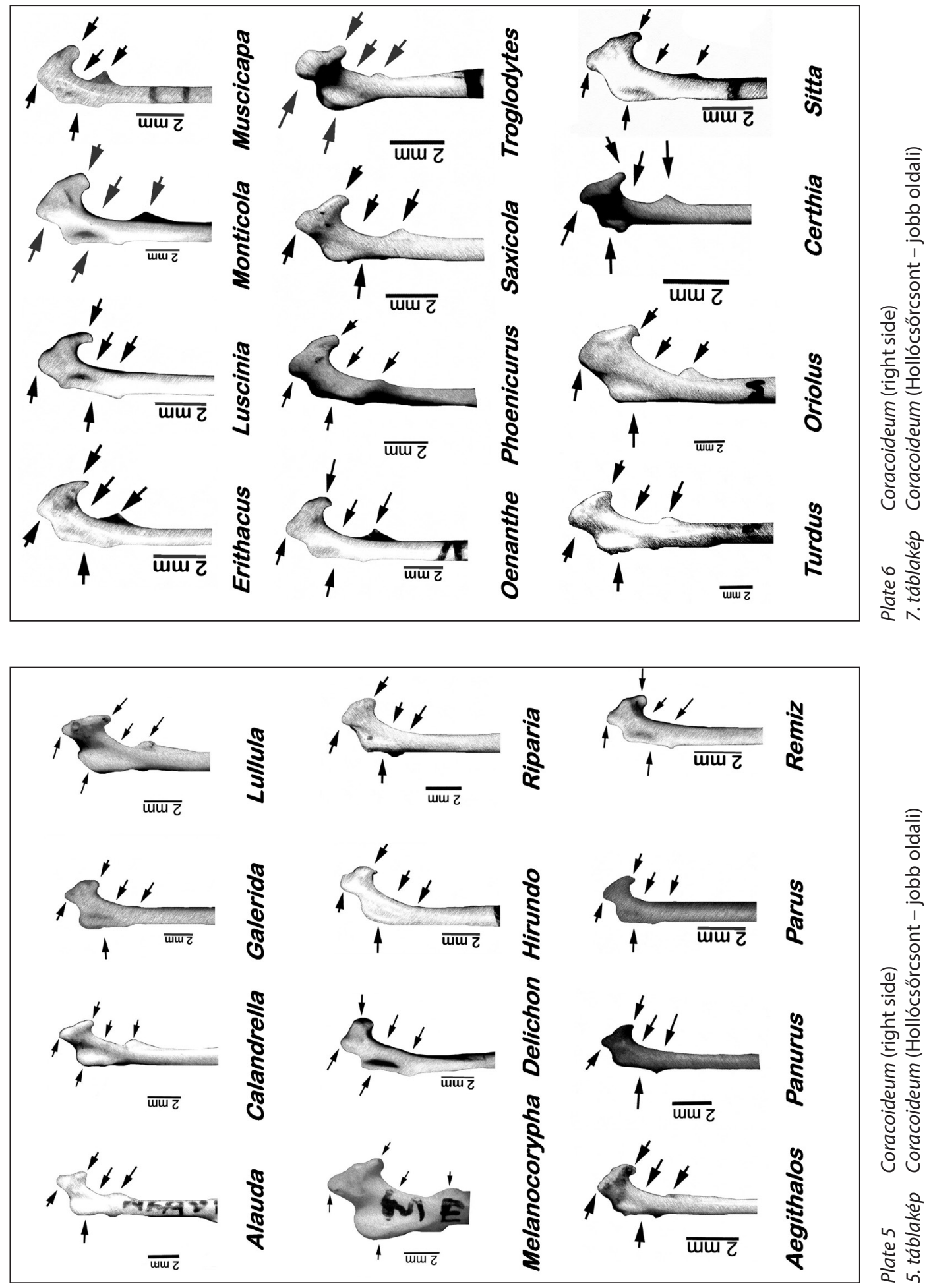


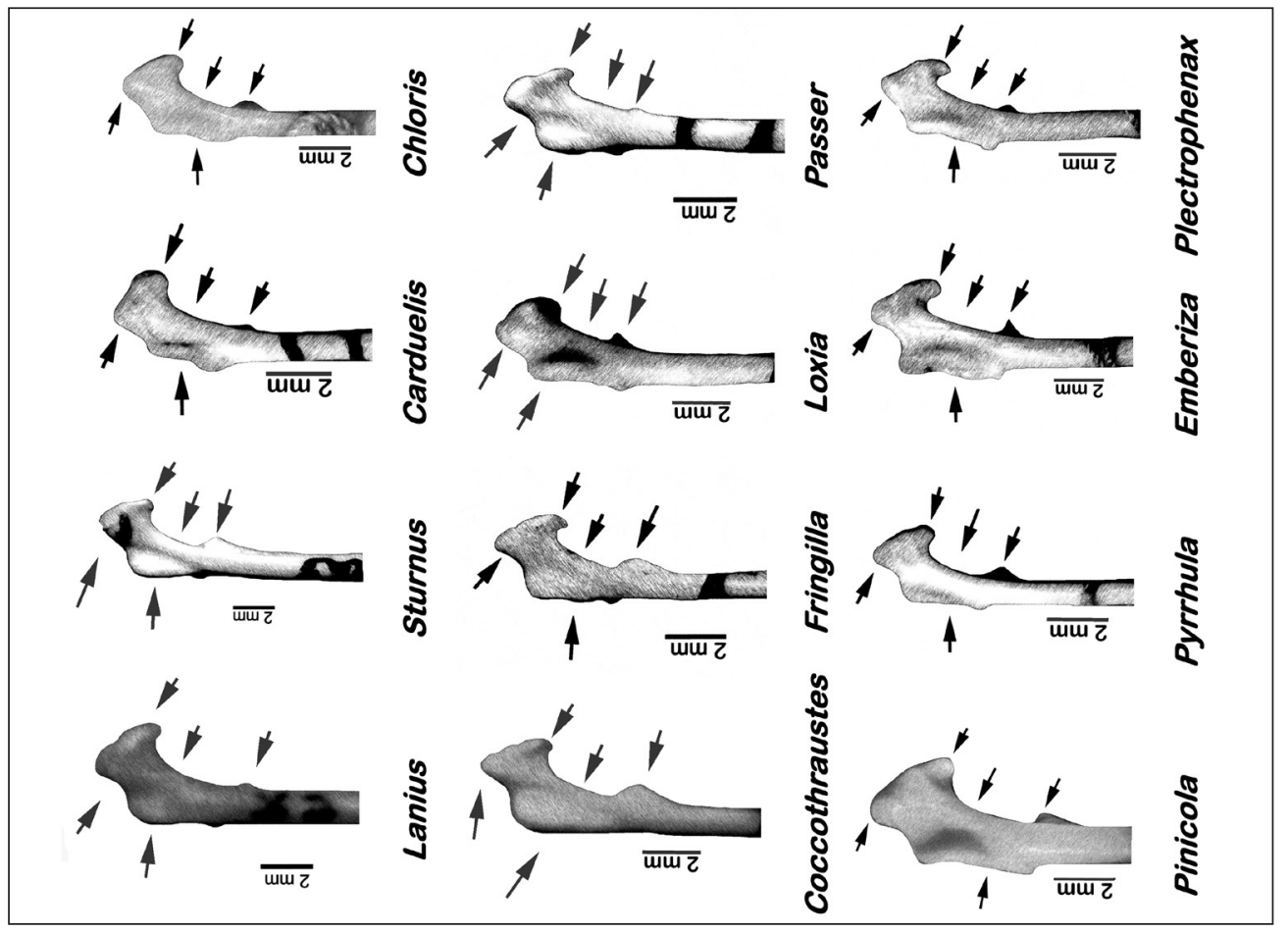

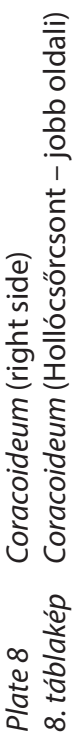

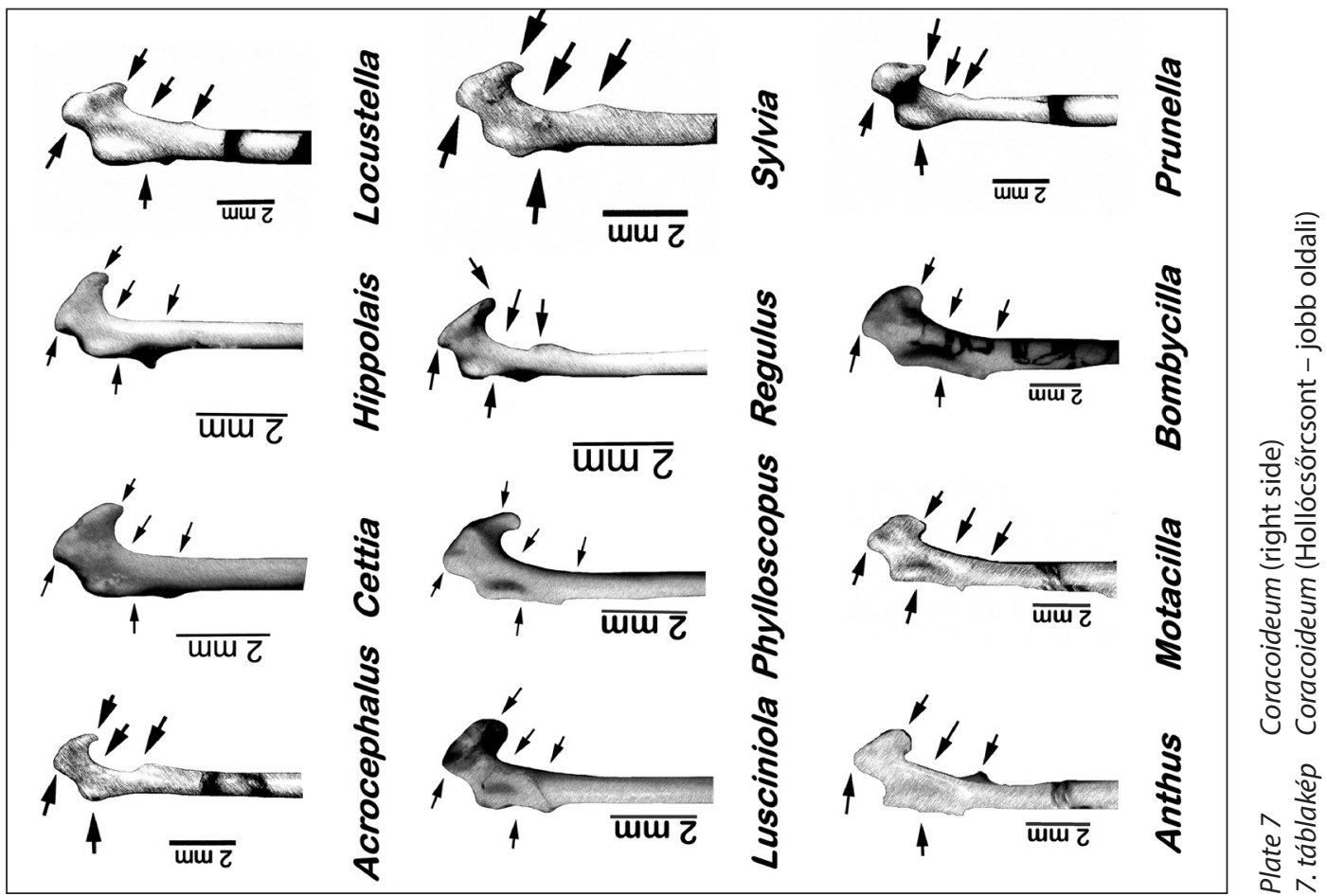



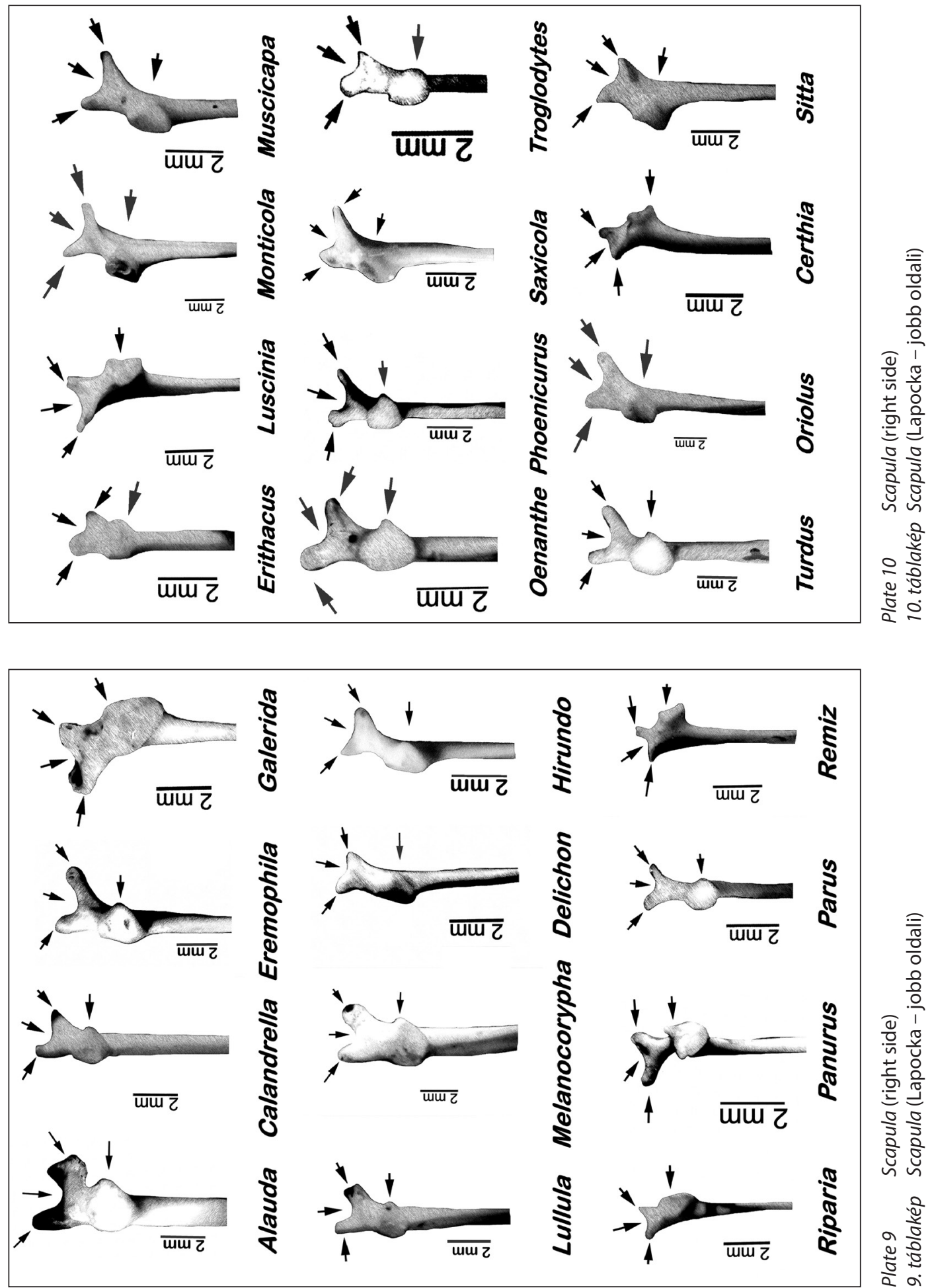


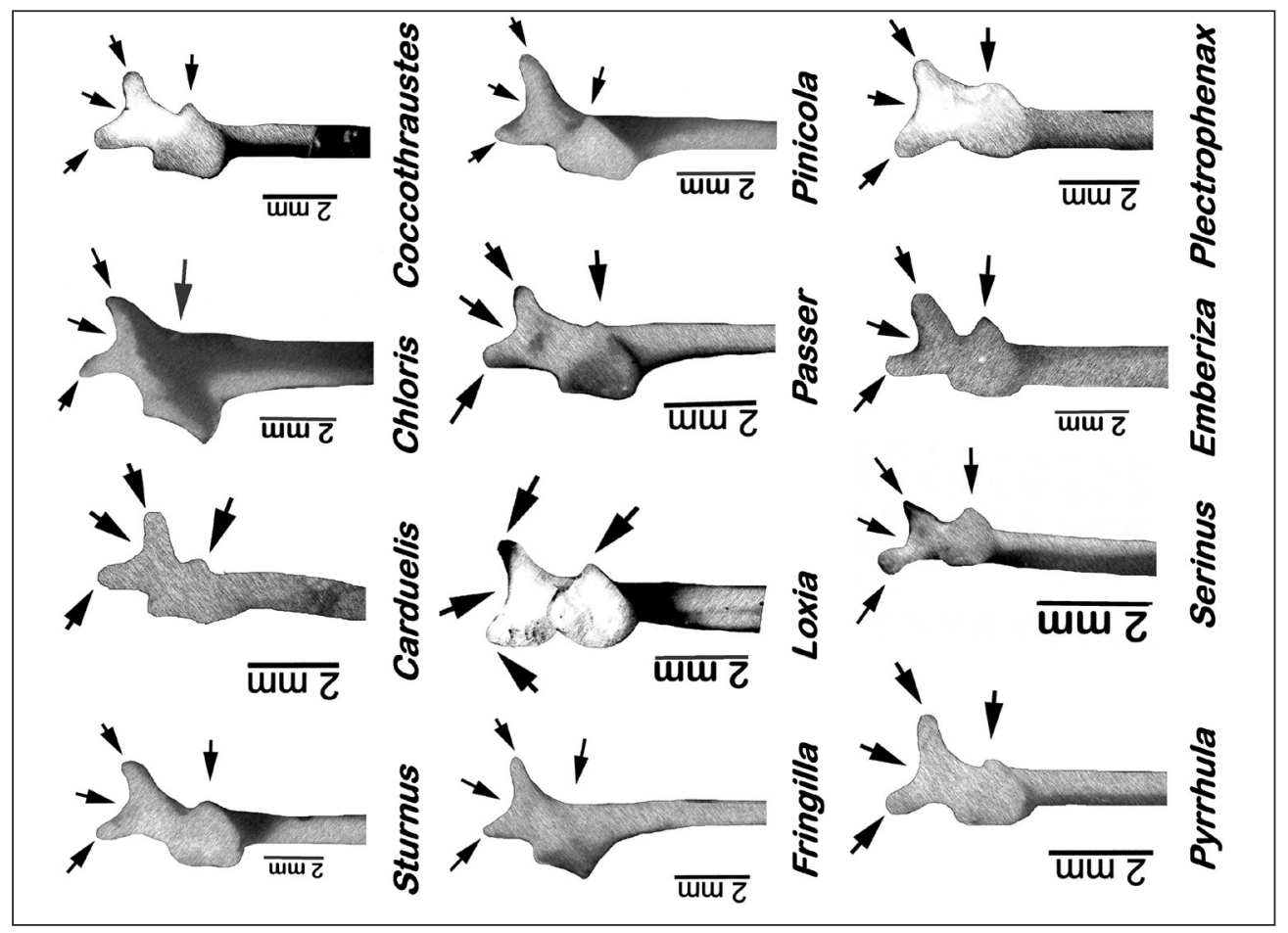

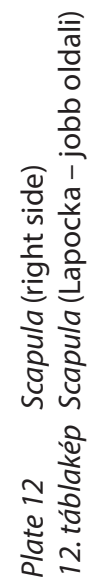

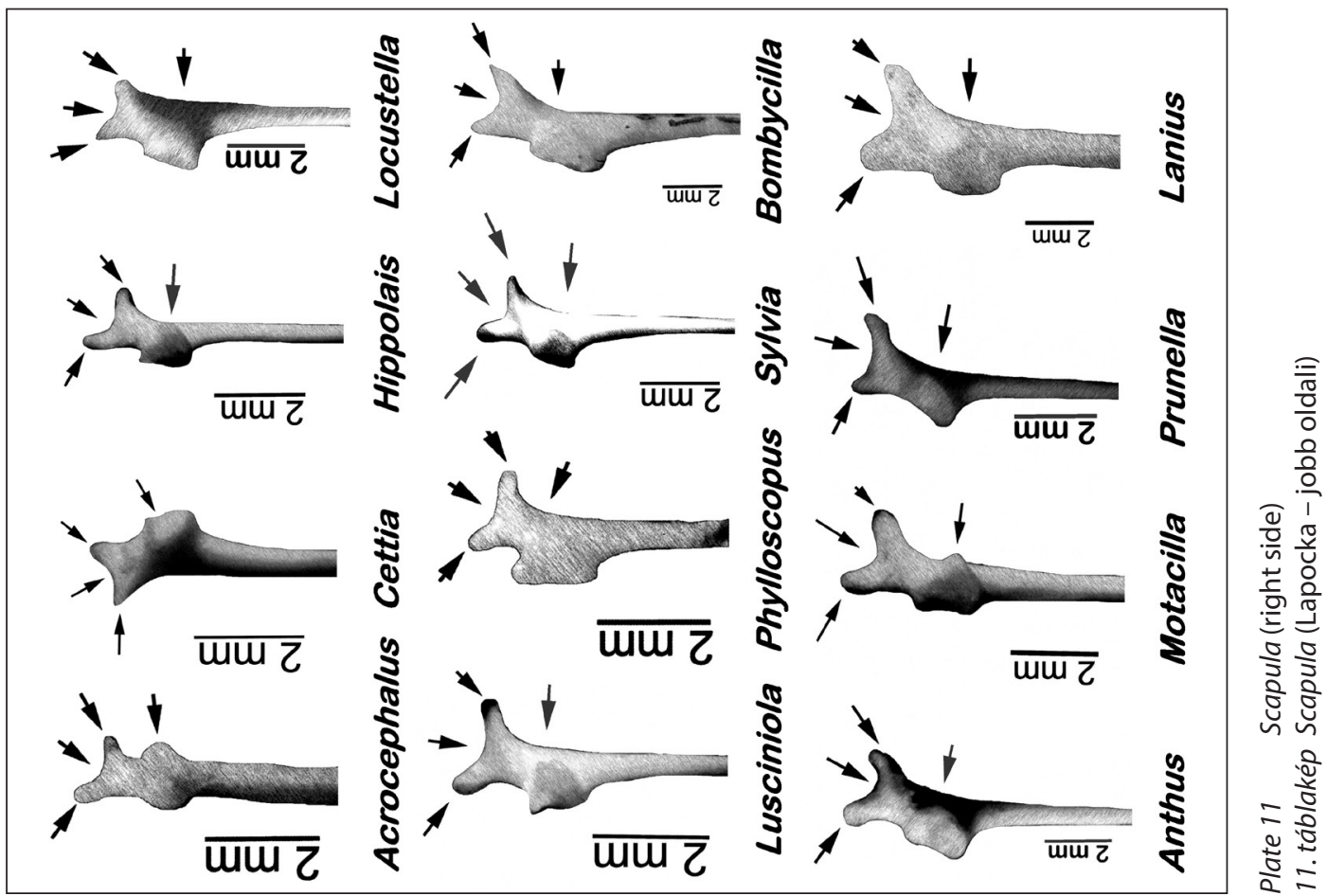



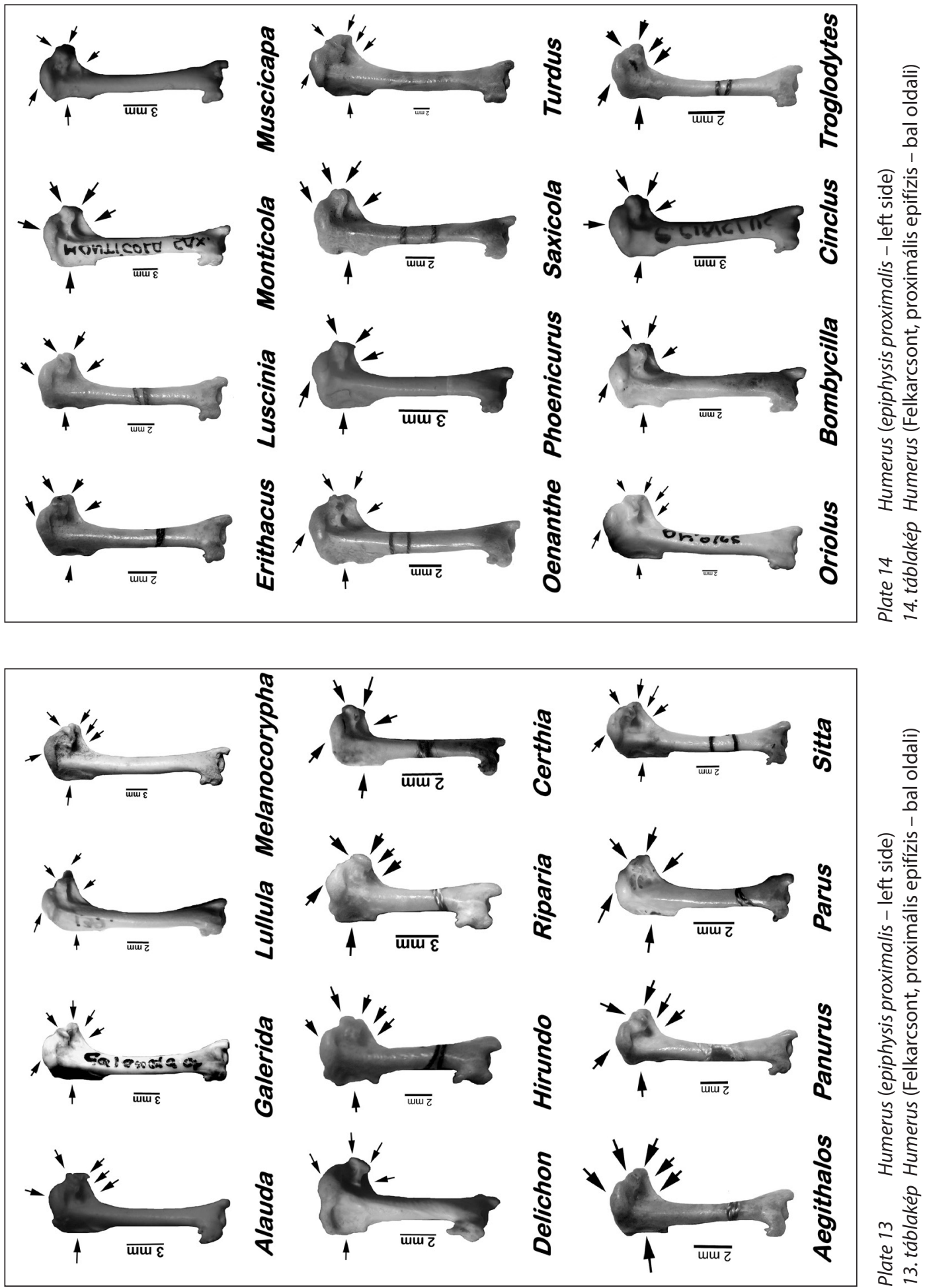

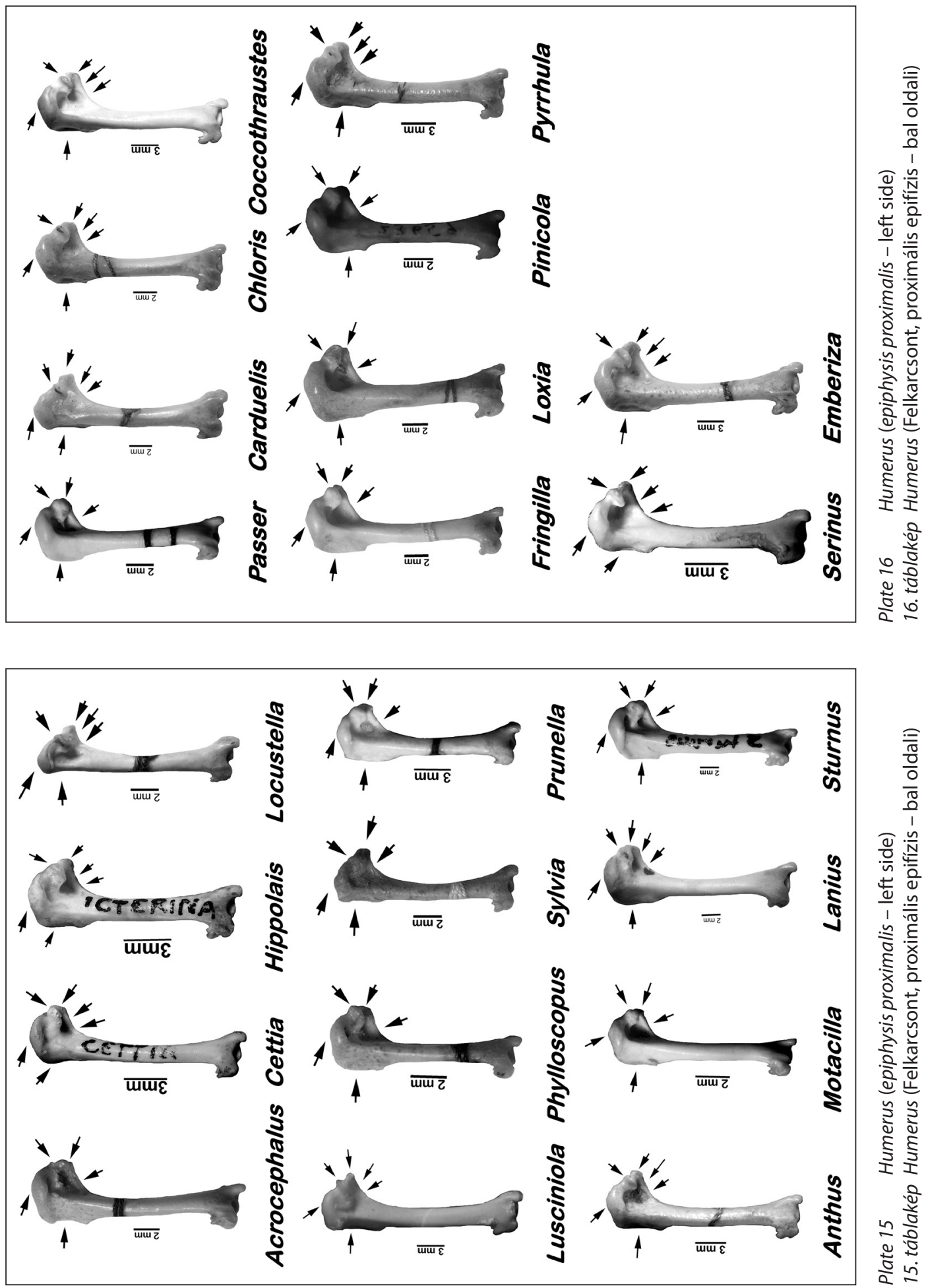

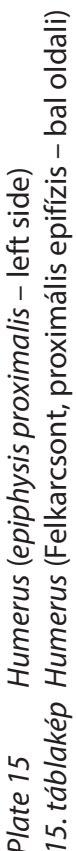



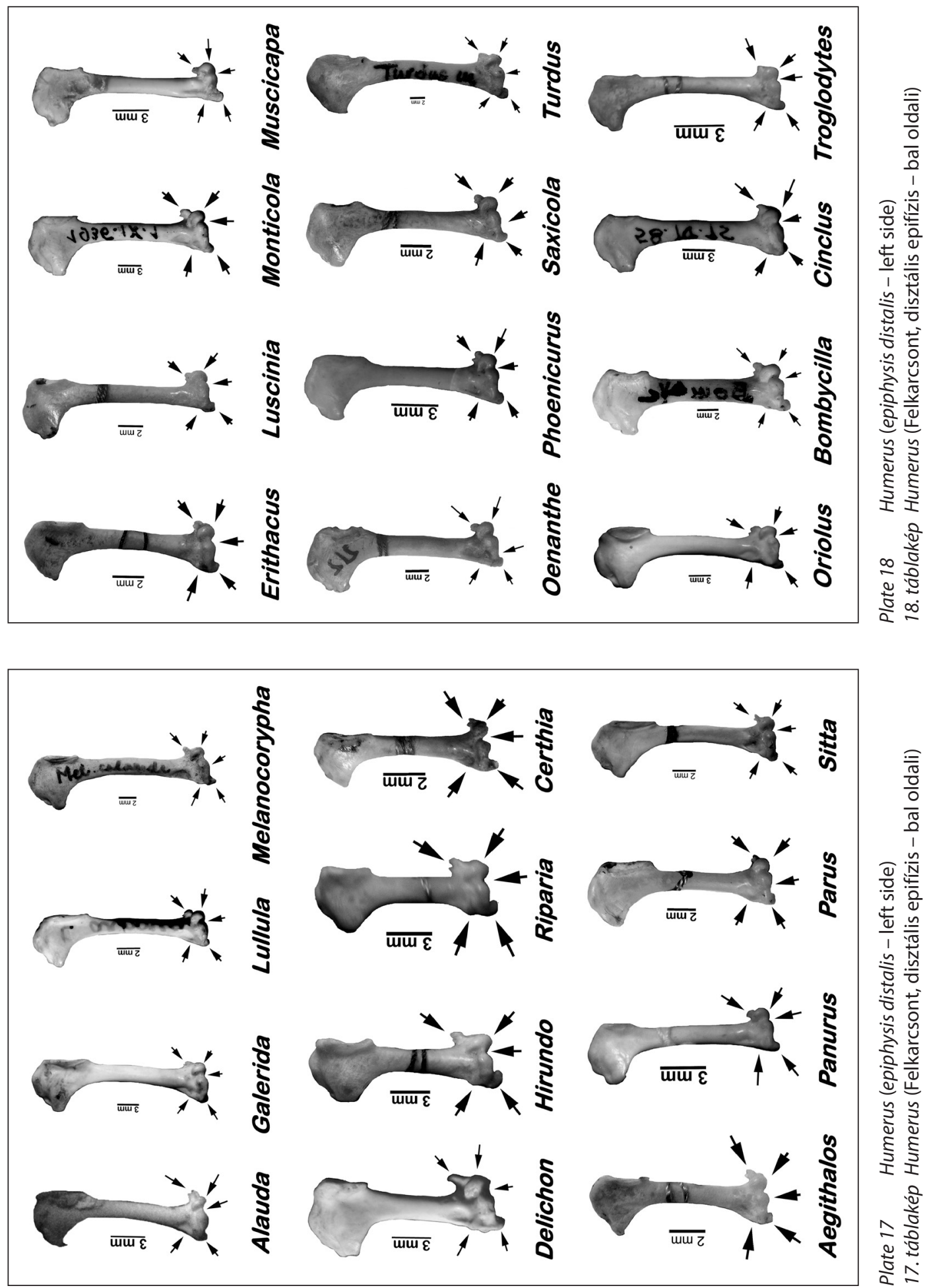

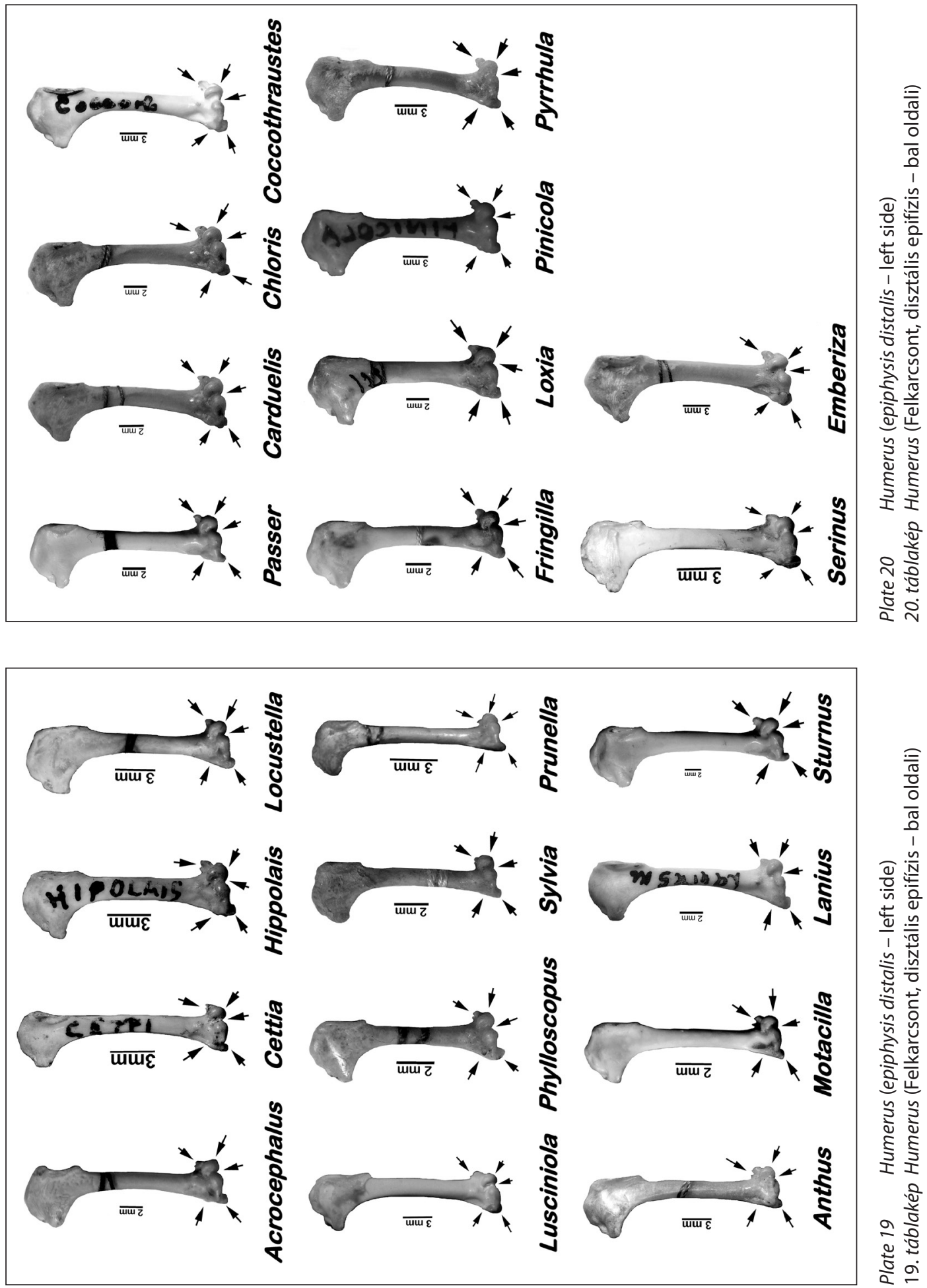

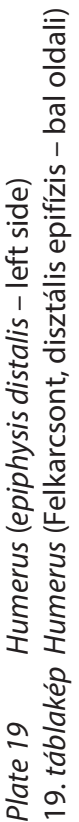



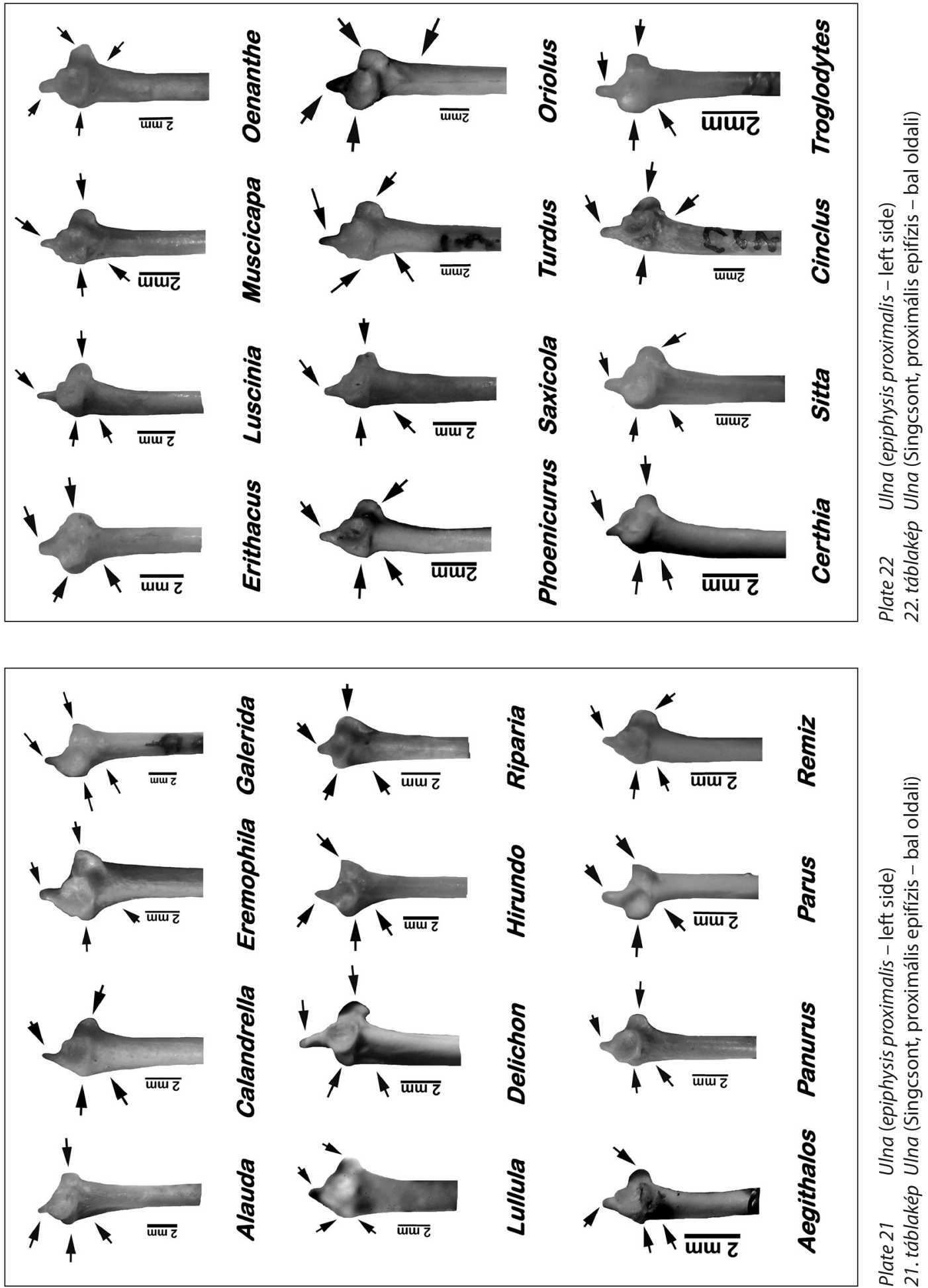

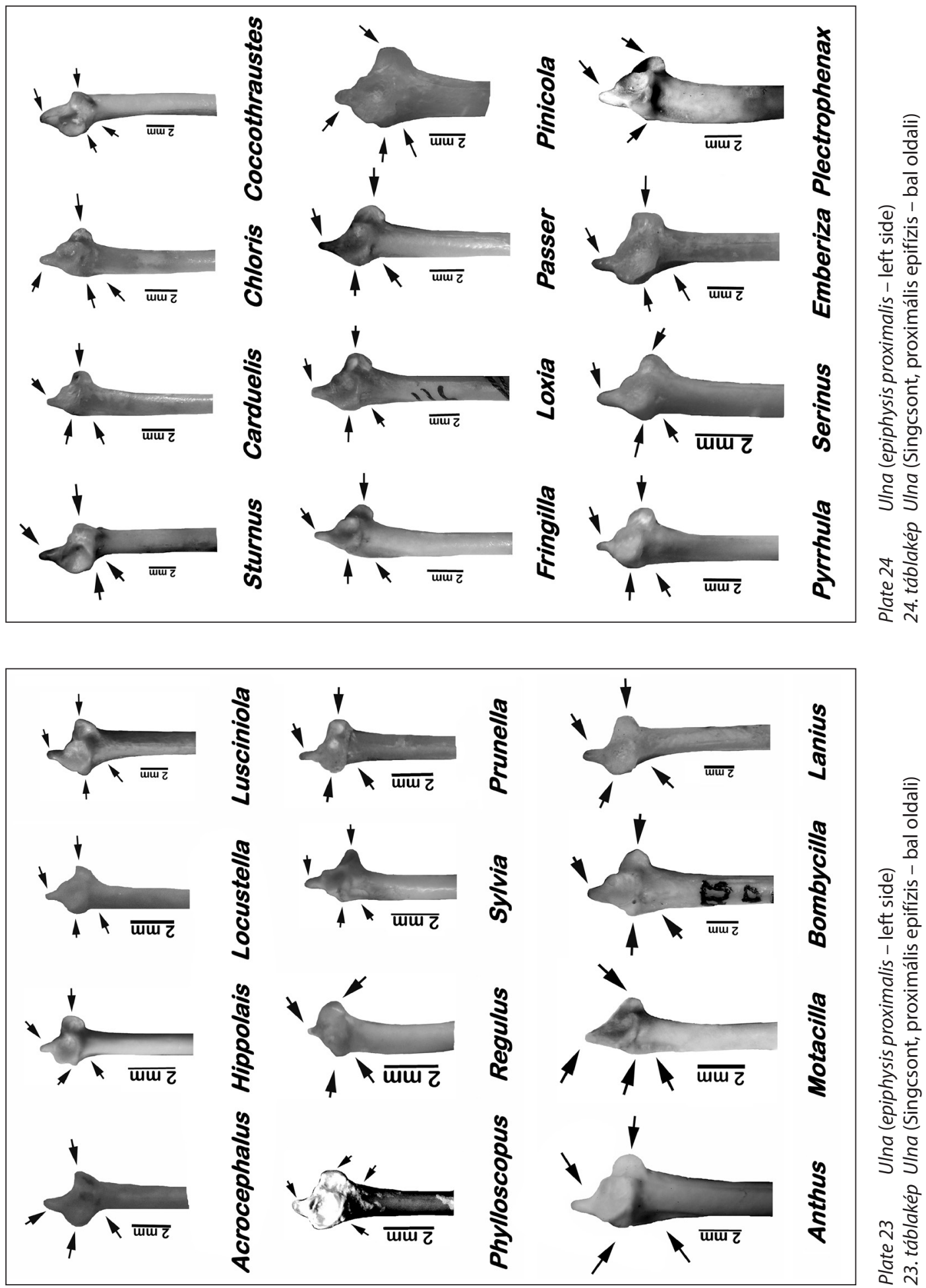

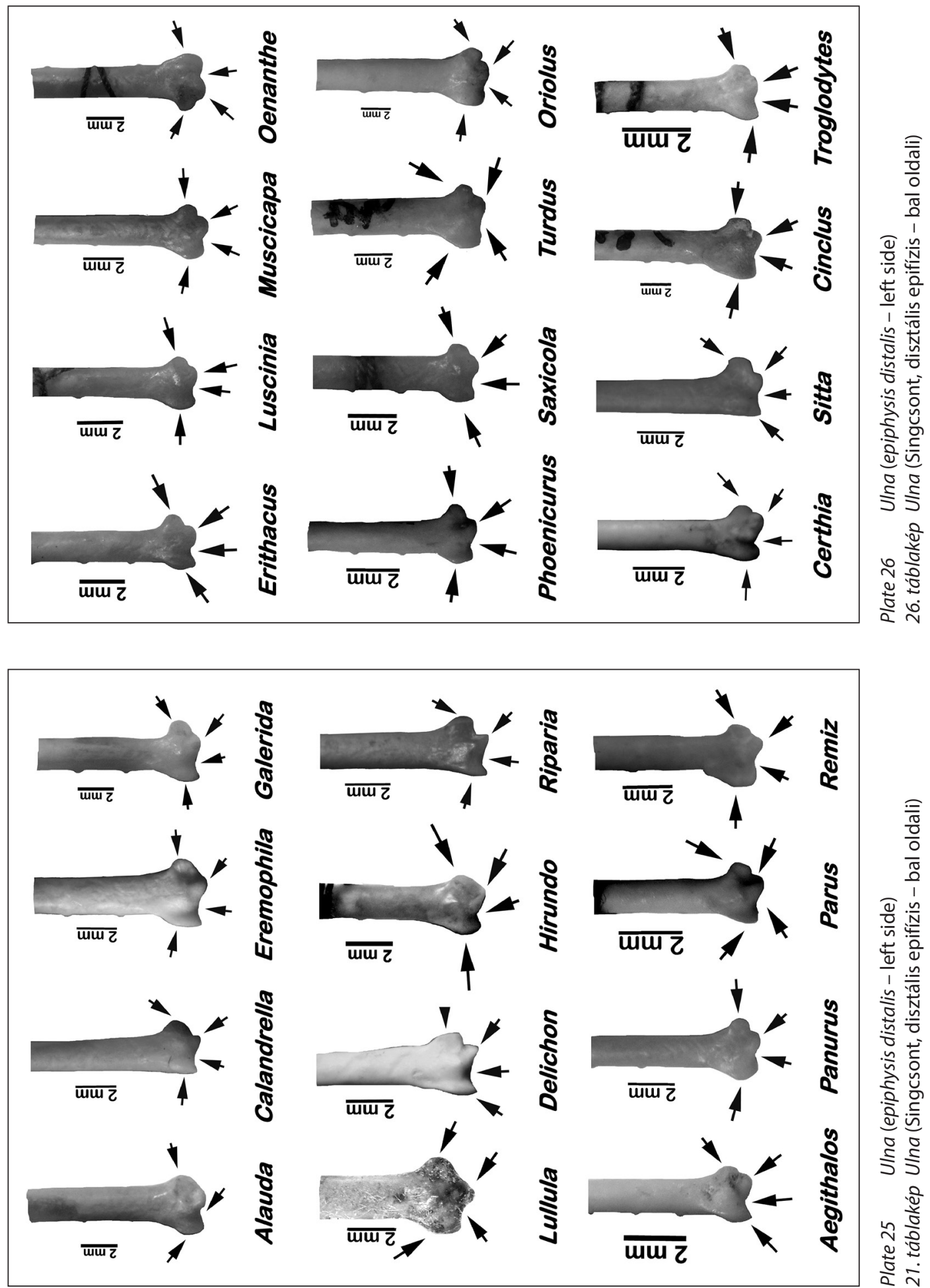


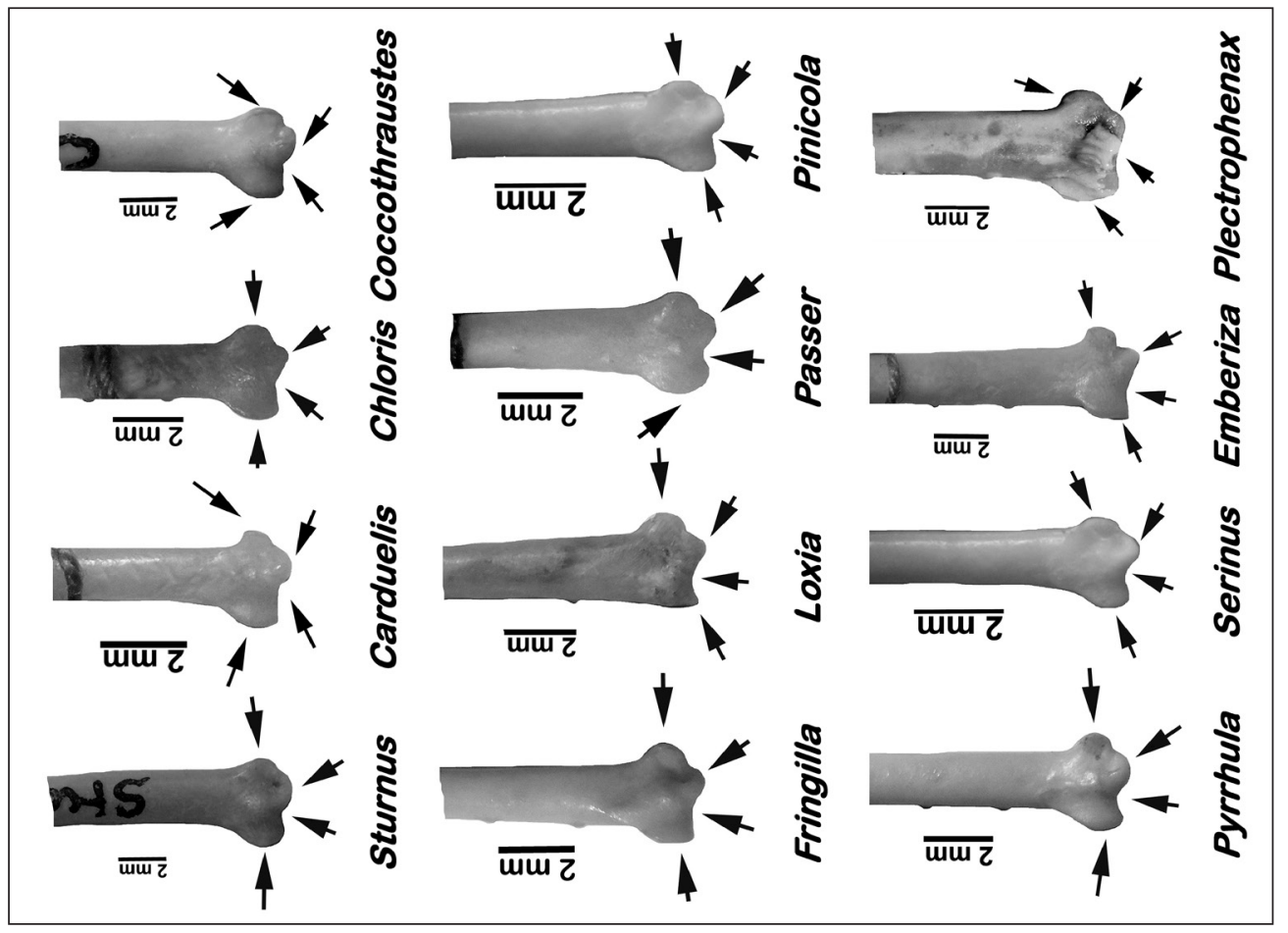

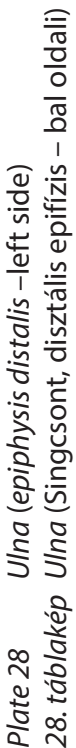

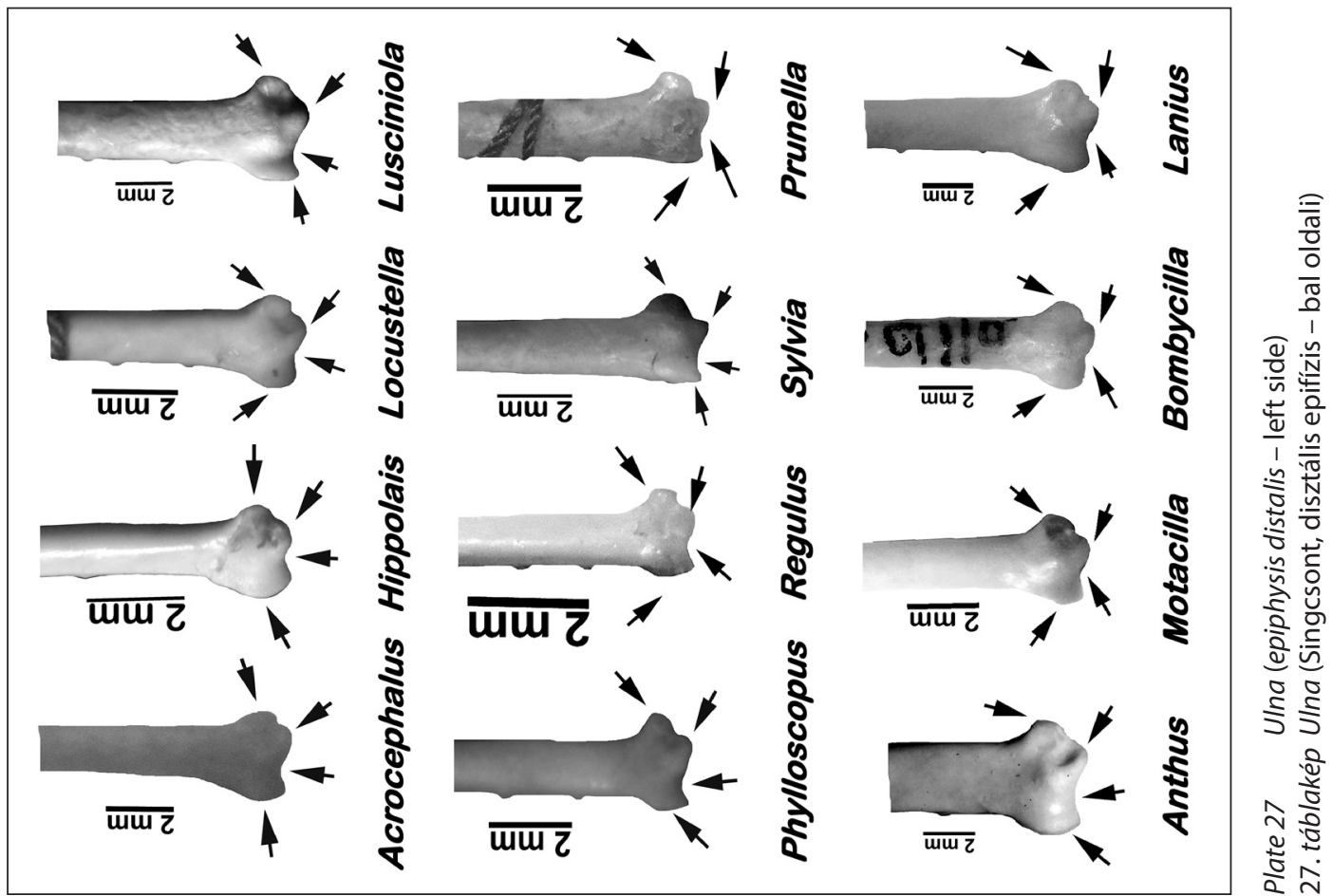



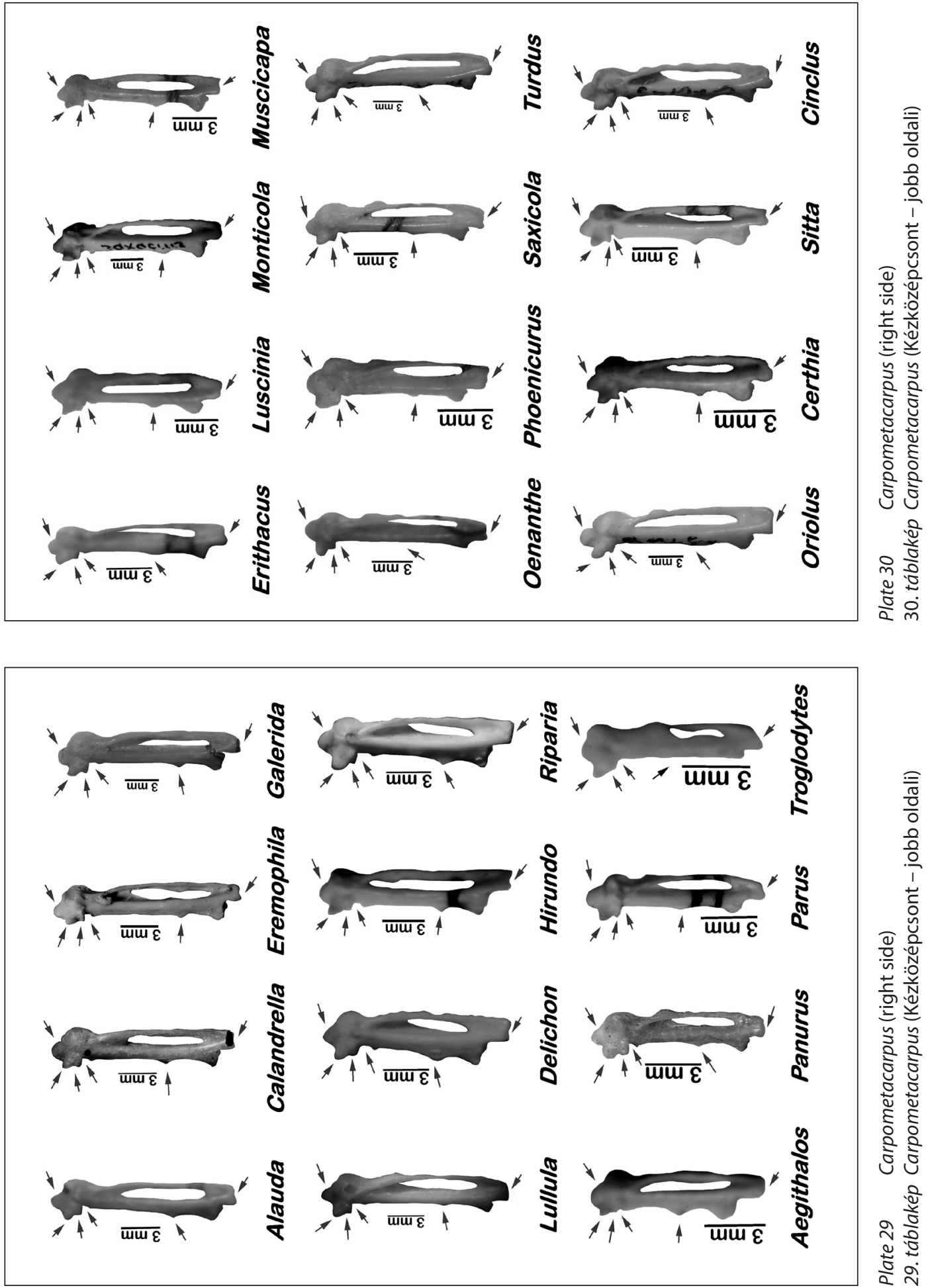

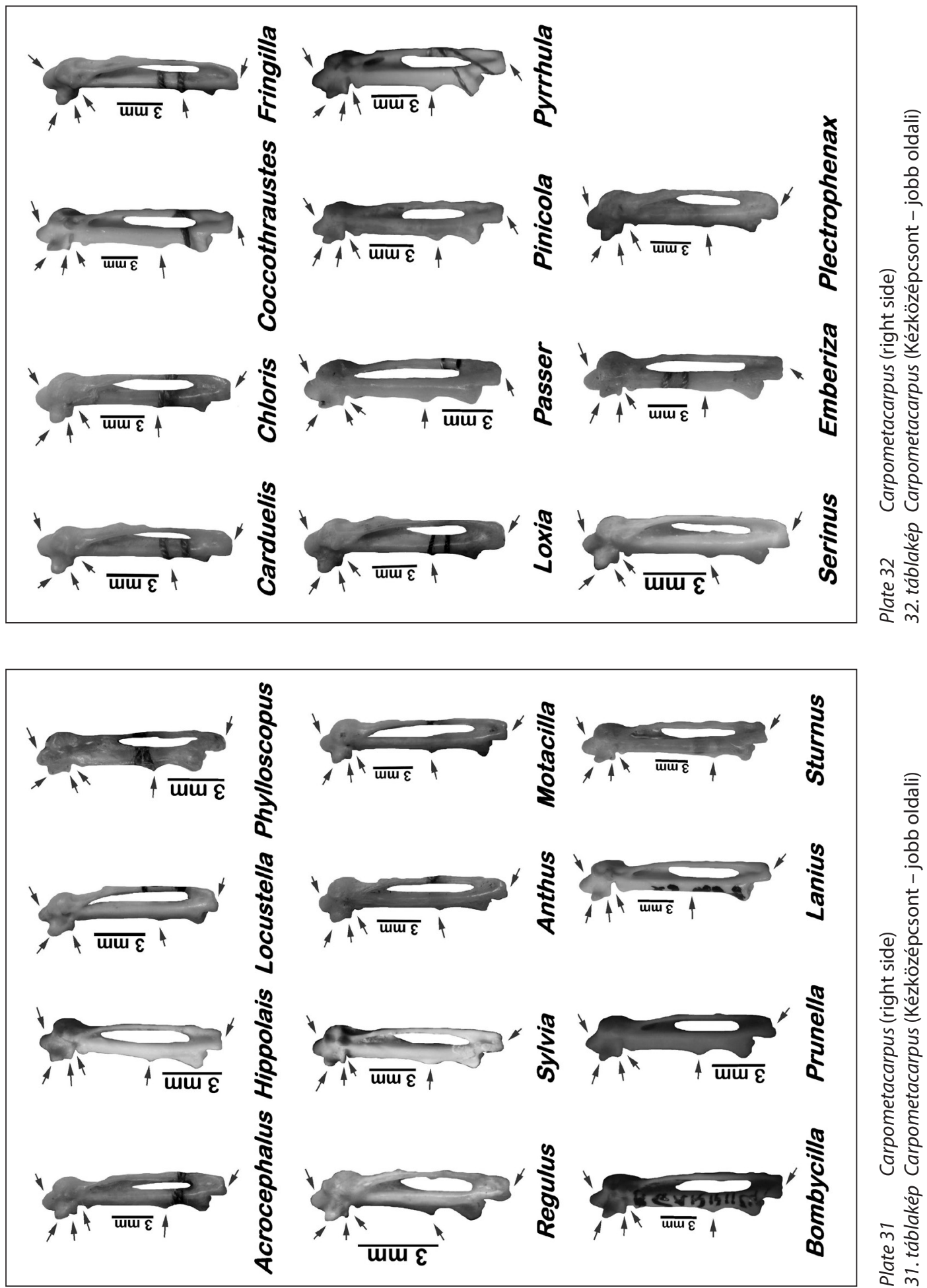

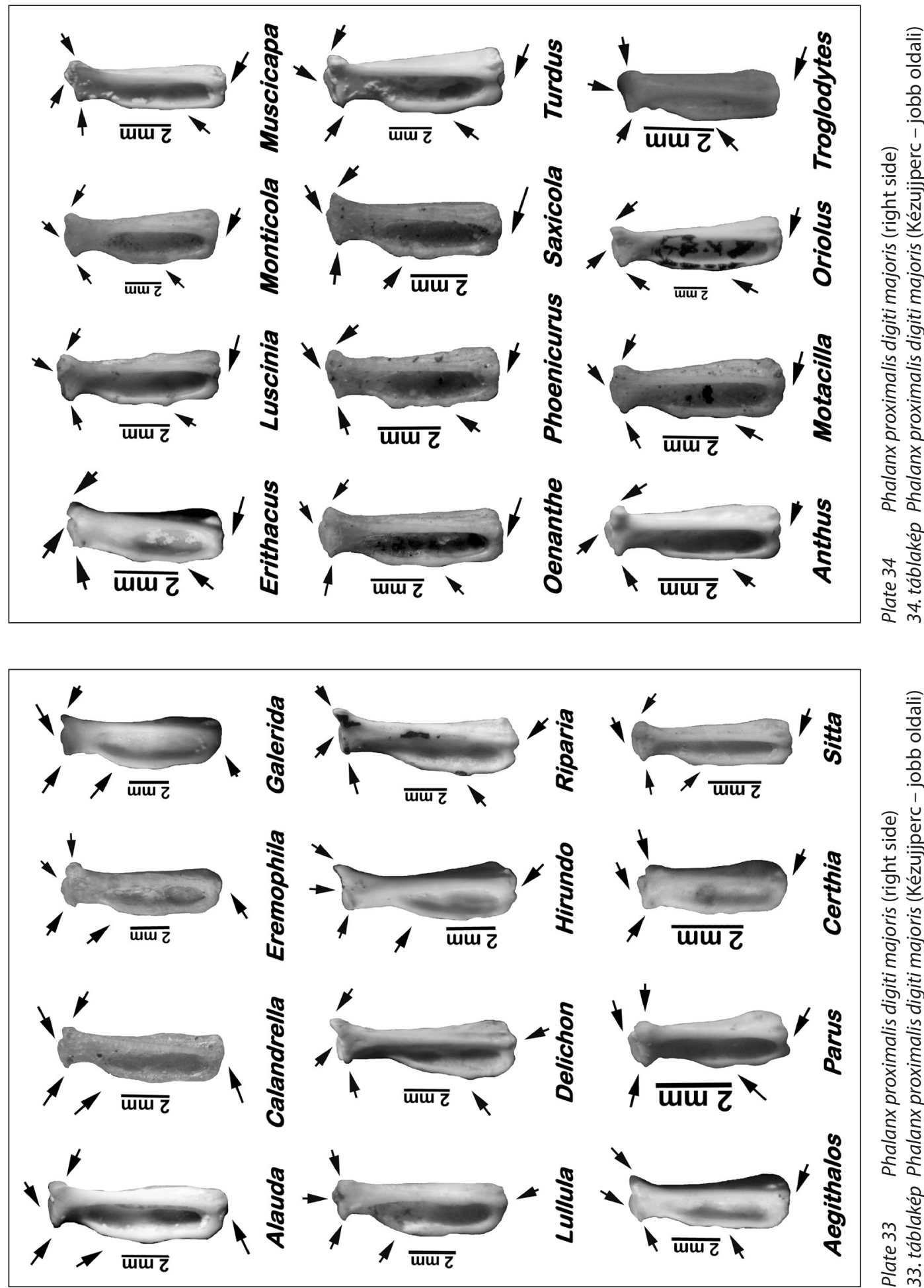

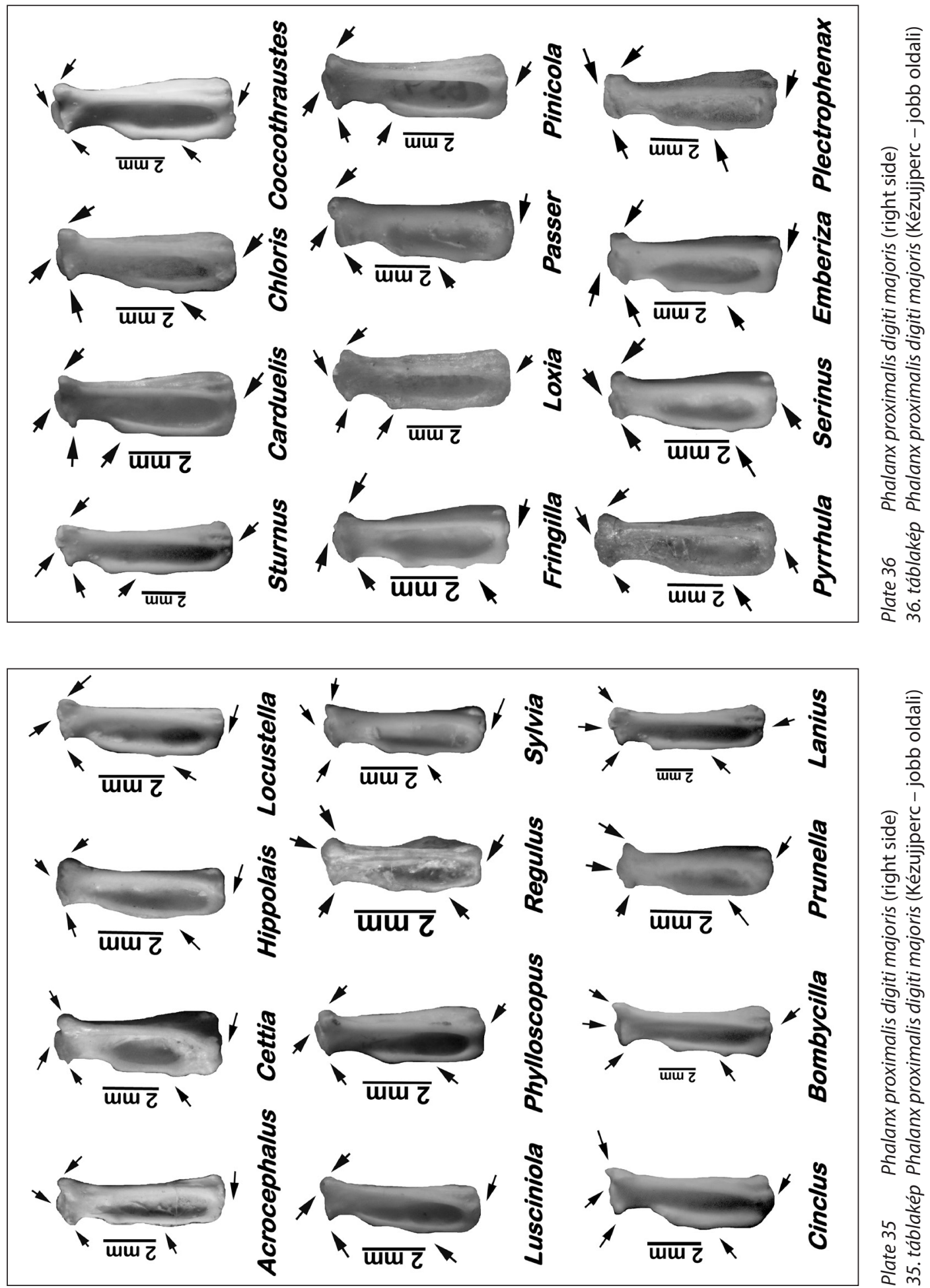

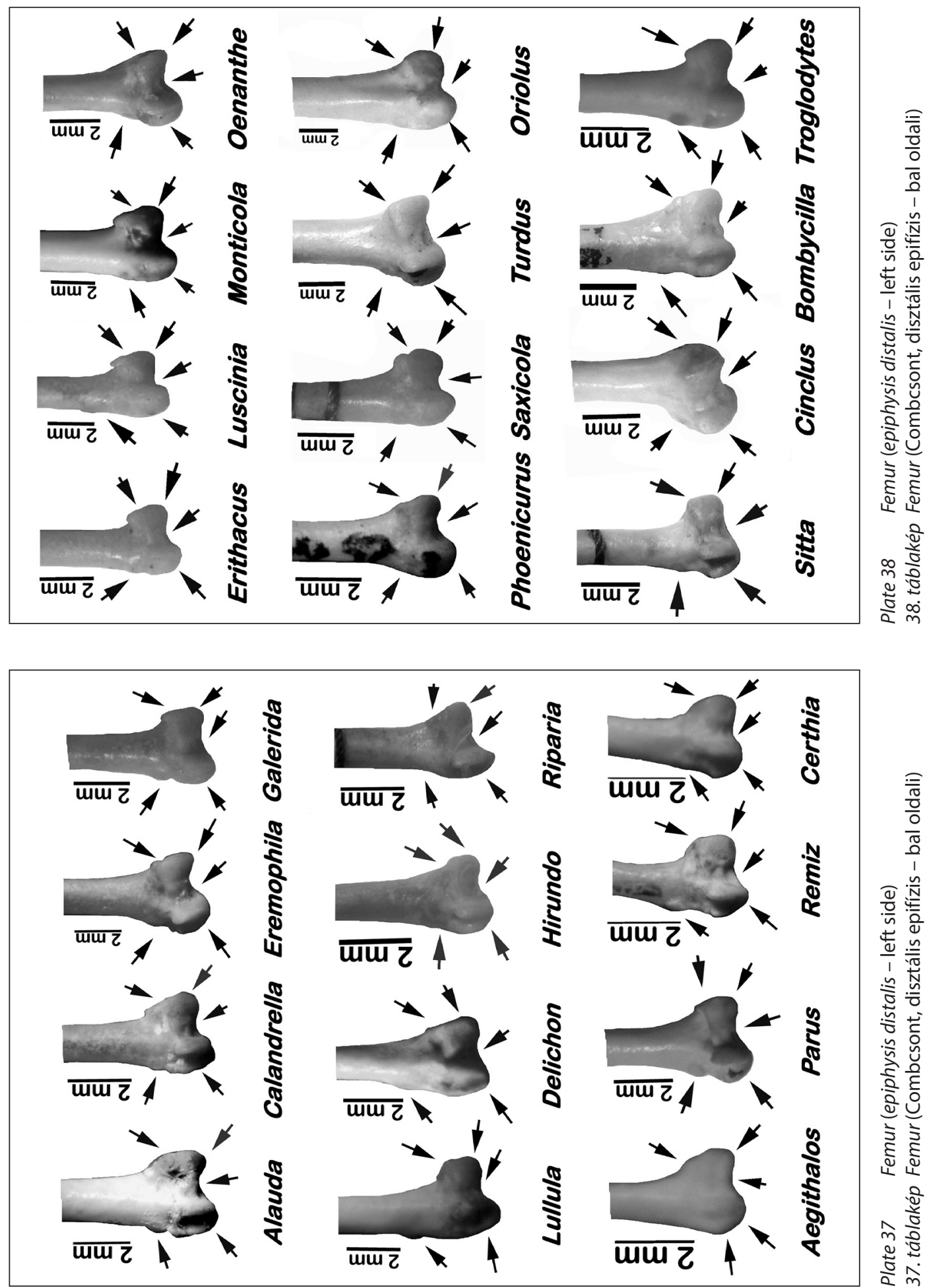

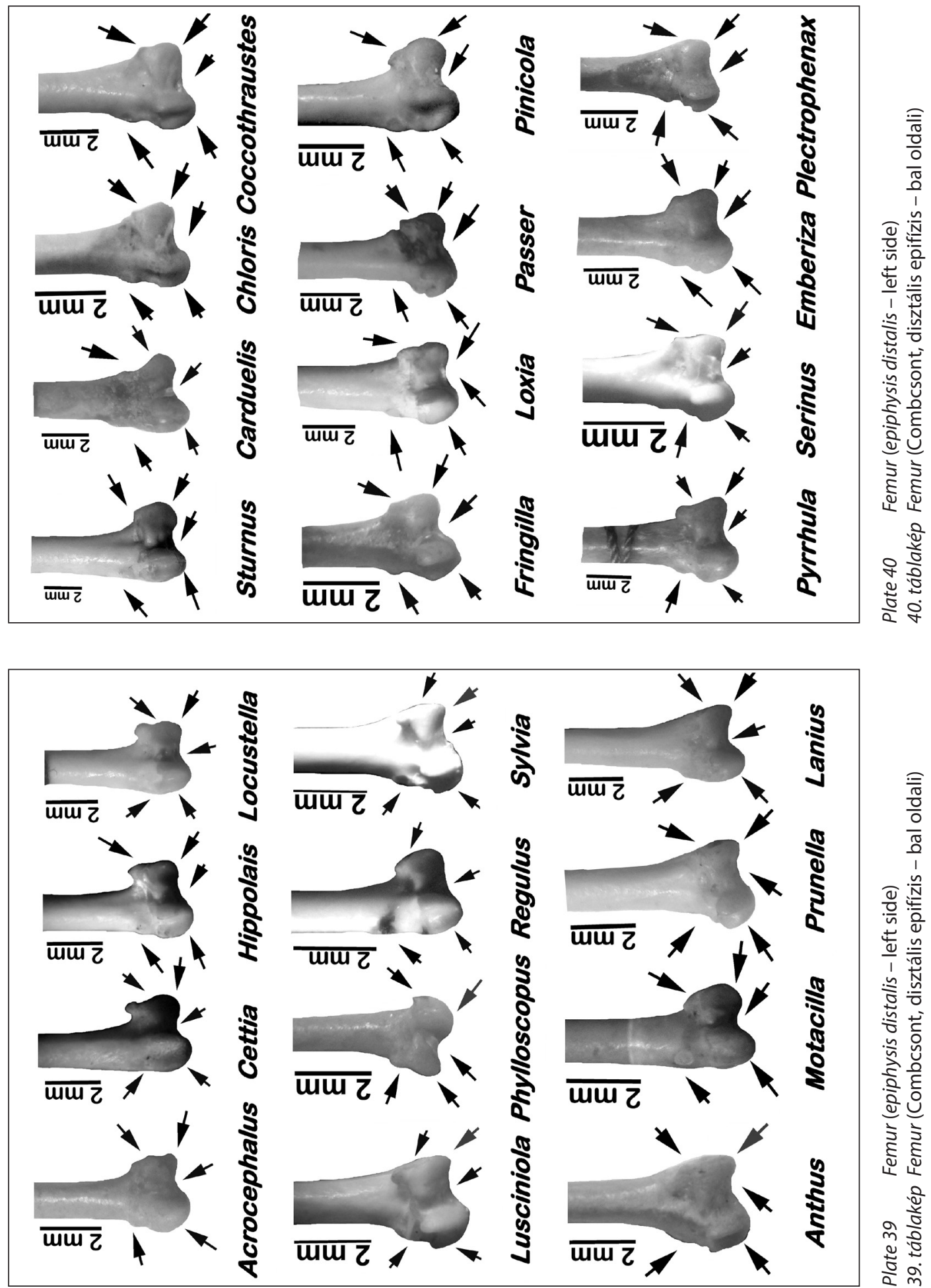

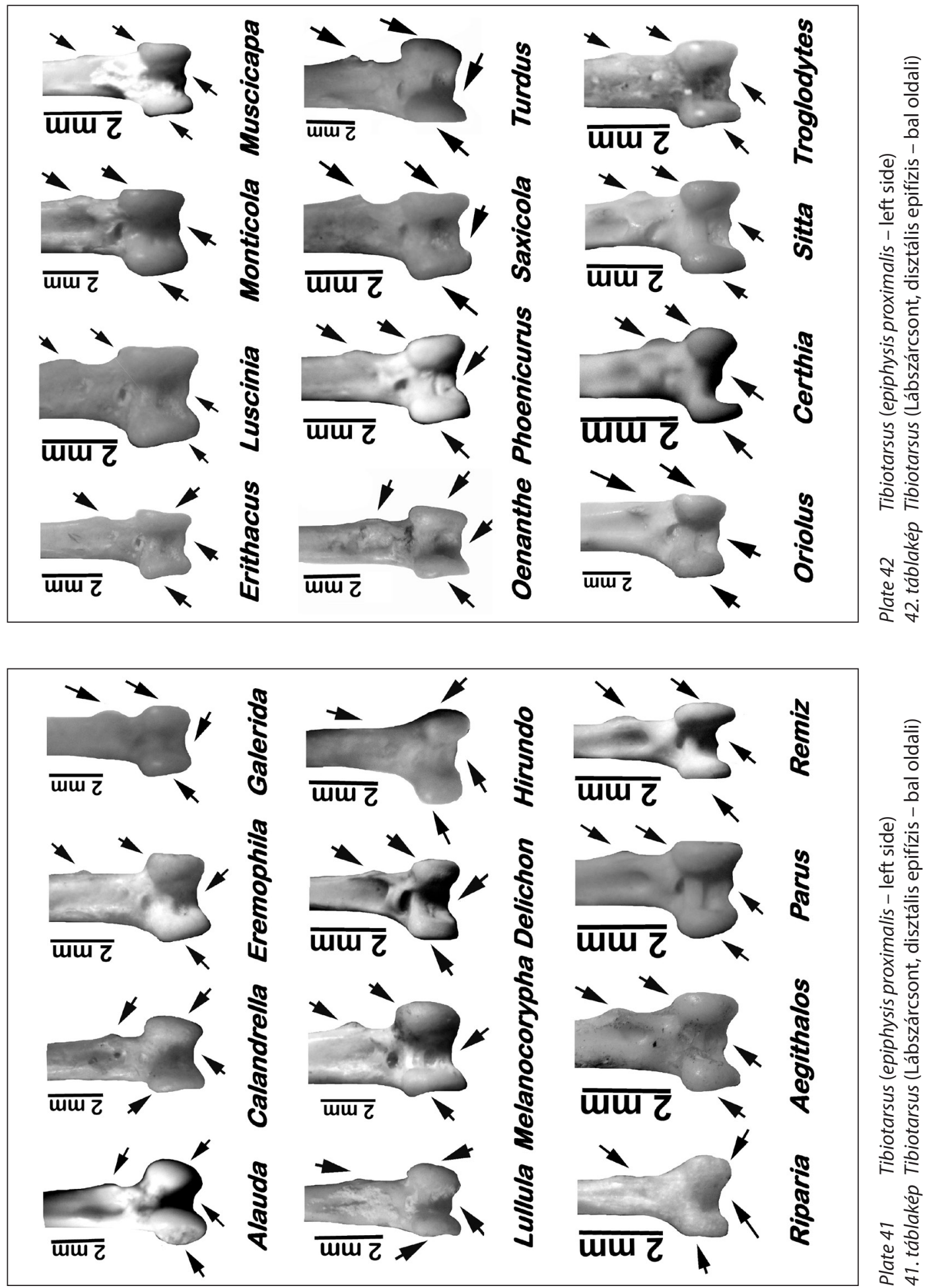

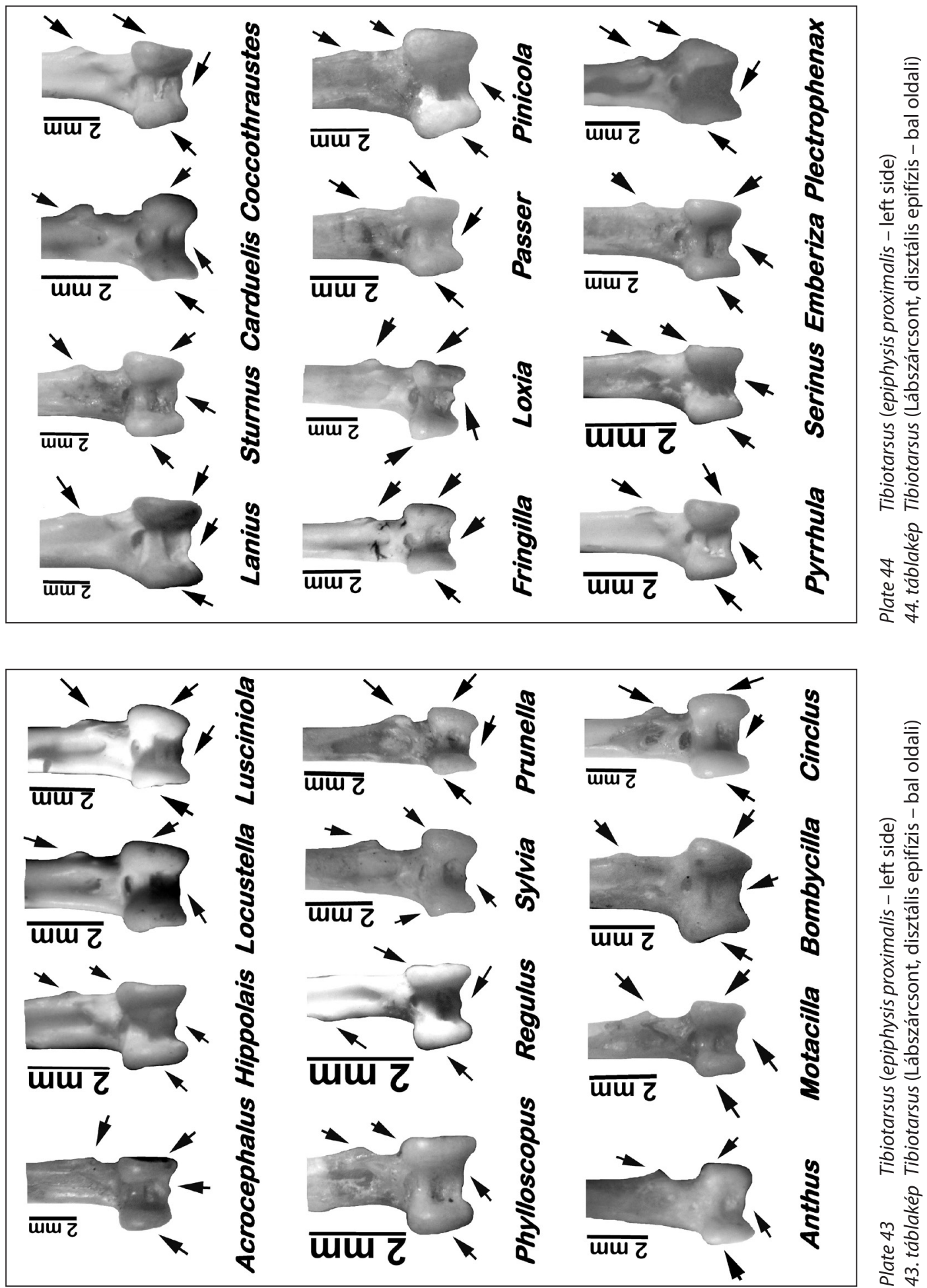

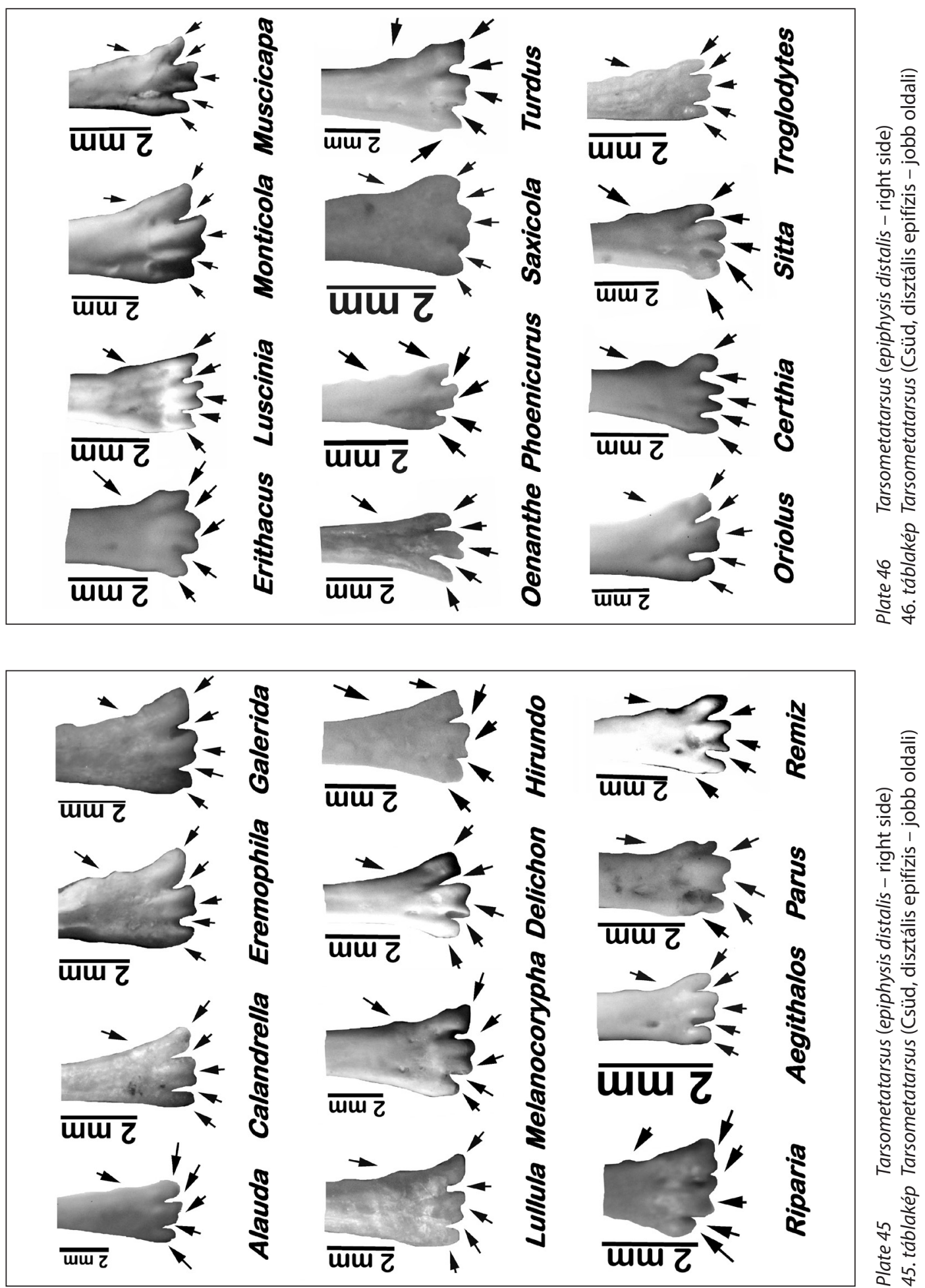


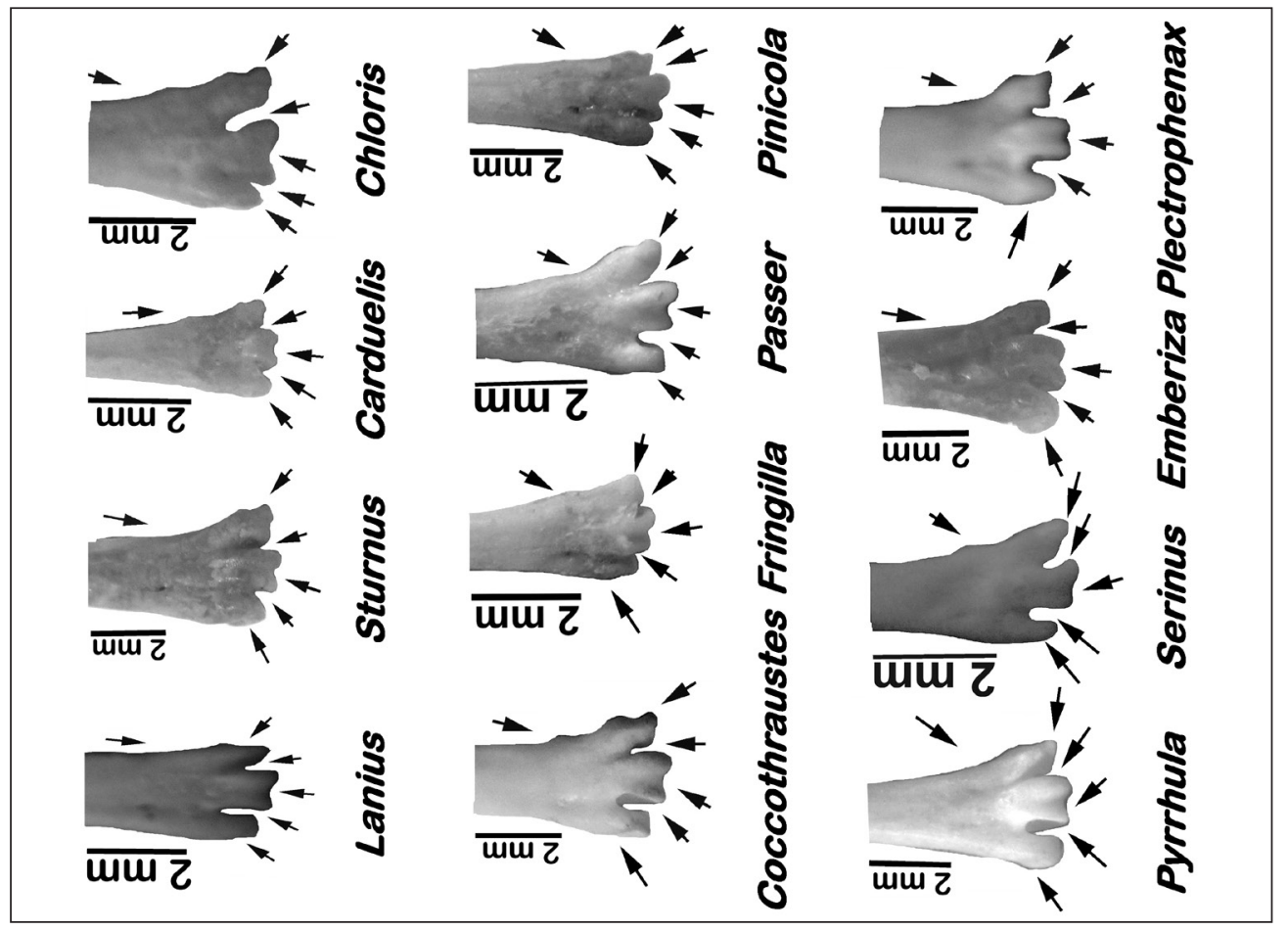

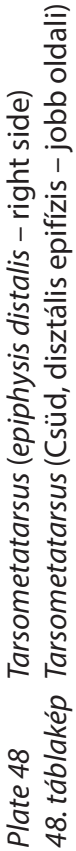

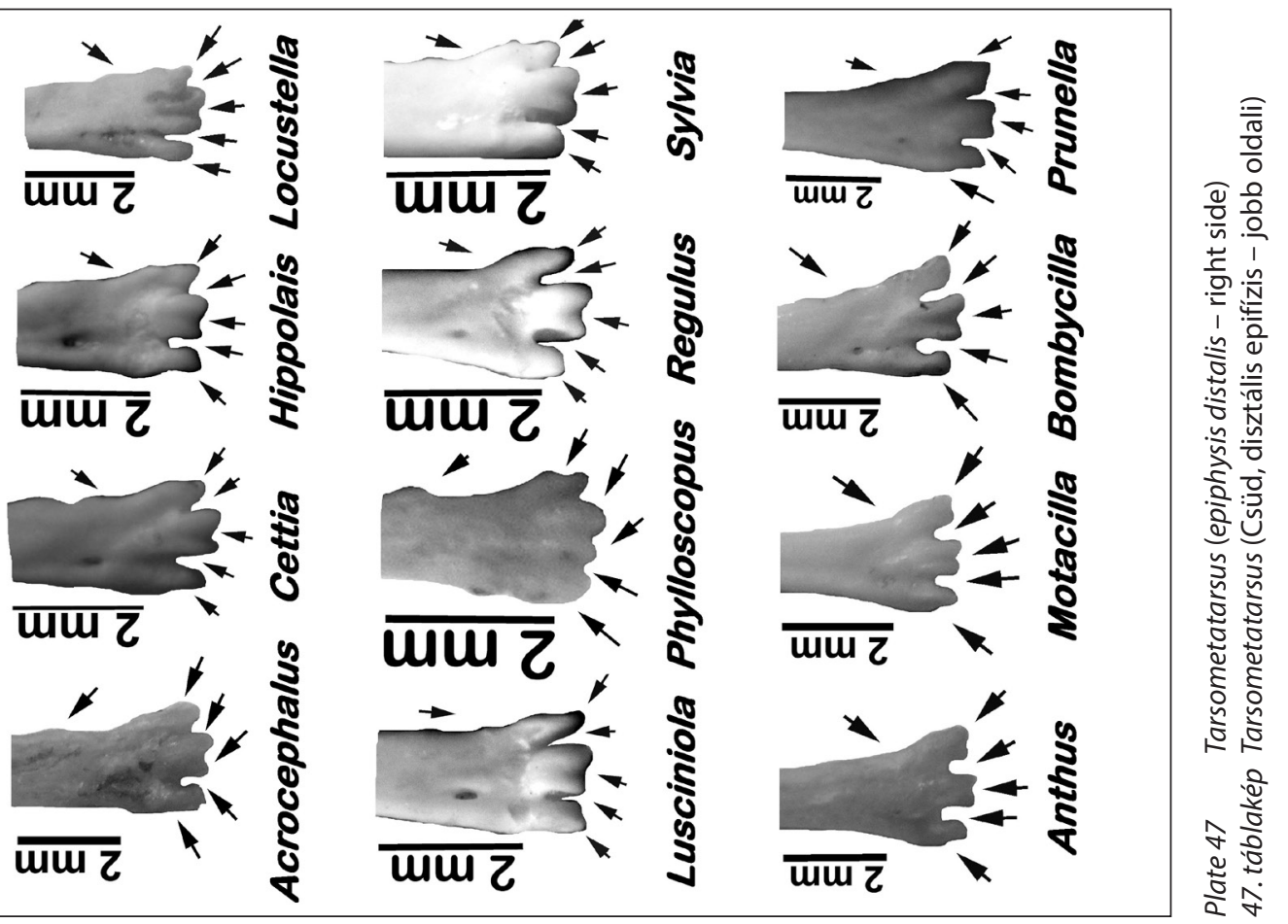



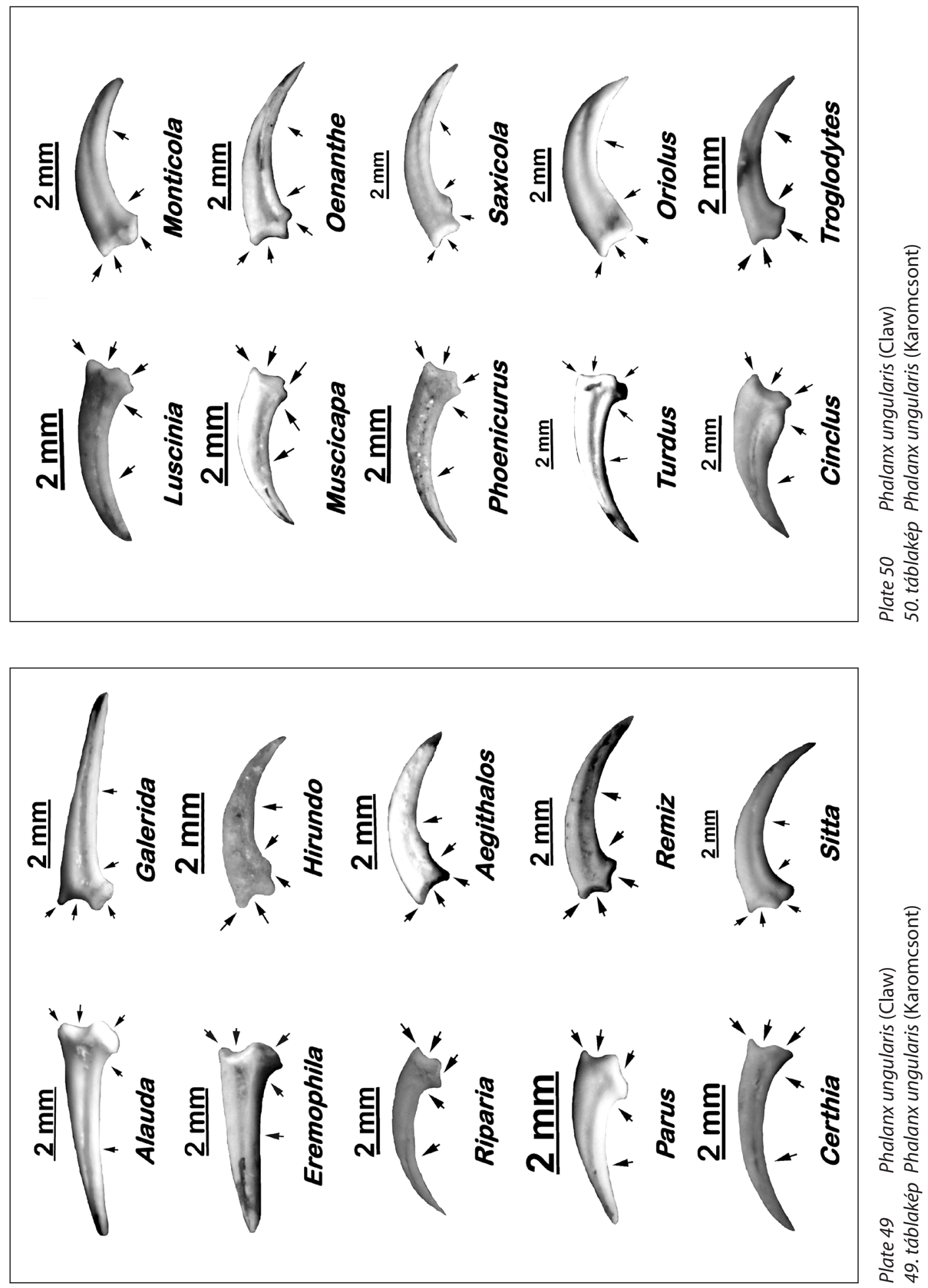


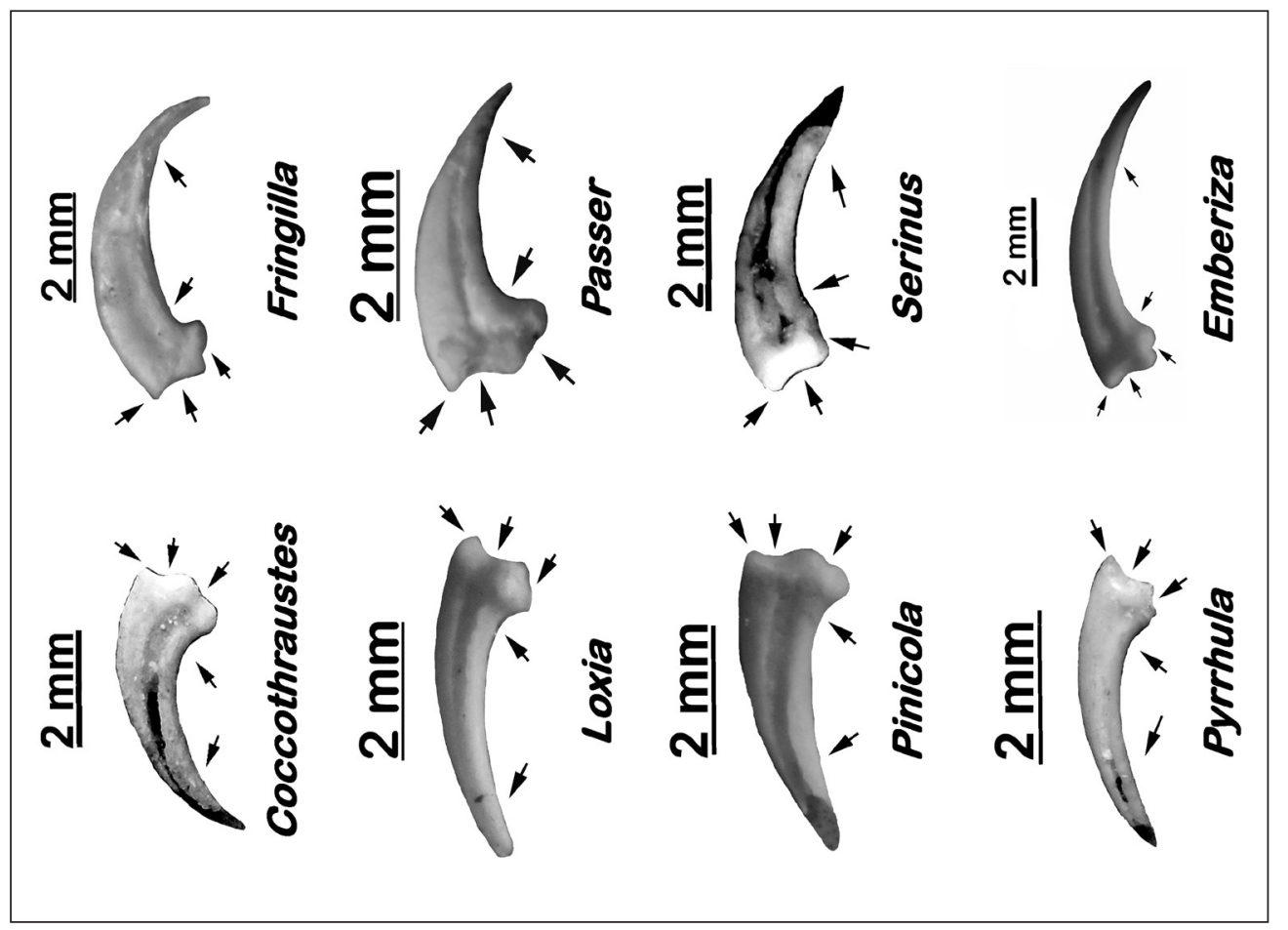

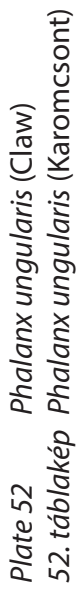

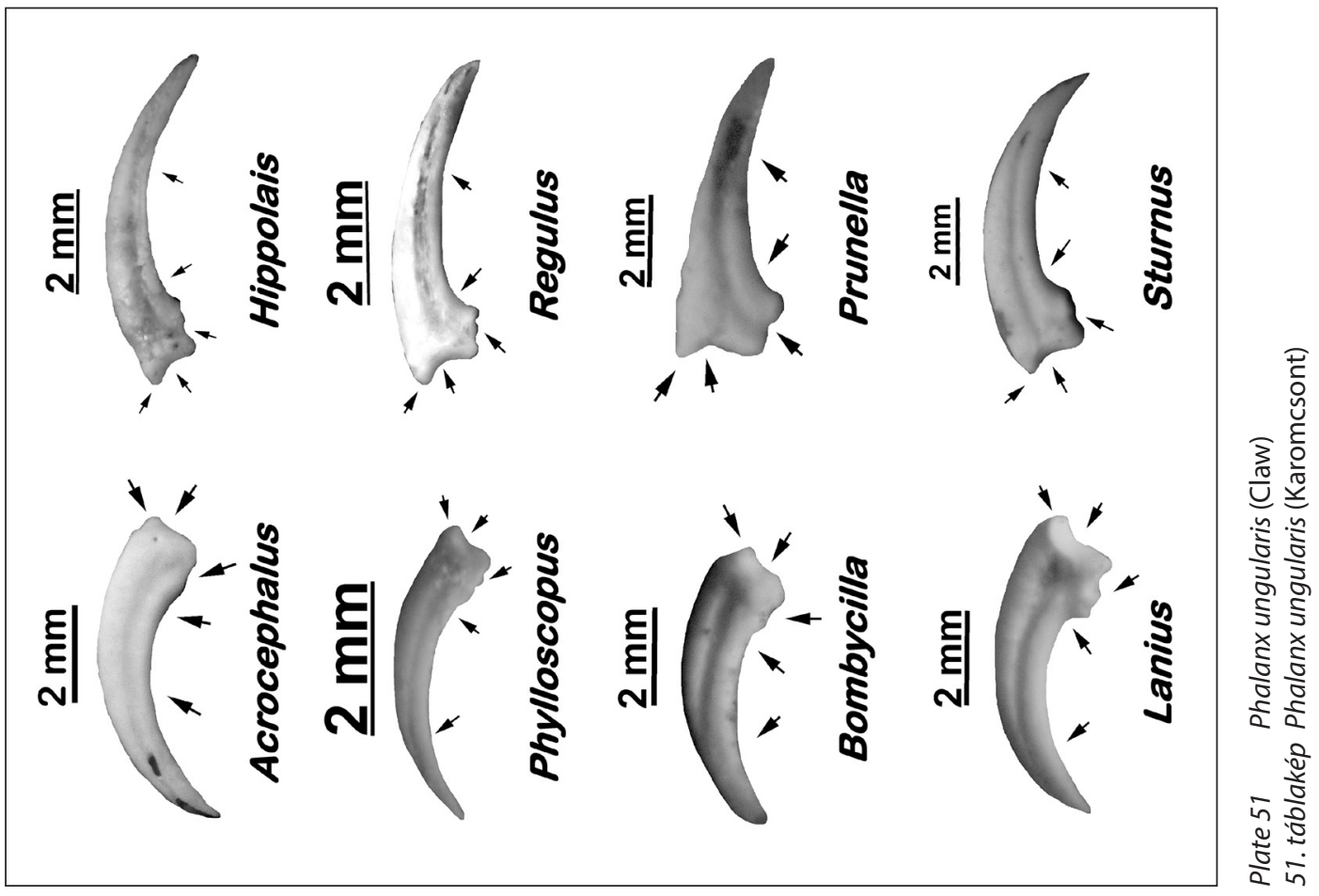

